LEONARDO SHIMIZU YOJO

ESTUDO, CARACTERIZAÇÃO ELÉTRICA E MODELAGEM DE TRANSISTORES BE (BACK ENHANCED) SOI MOSFET 


\section{ESTUDO, CARACTERIZAÇÃO ELÉTRICA E MODELAGEM DE TRANSISTORES BE (BACK ENHANCED) SOI MOSFET}

Dissertação apresentada à Escola Politécnica da Universidade de São Paulo como parte dos requisitos necessários para a obtenção do título de Mestre em Ciências. 


\section{ESTUDO, CARACTERIZAÇÃO ELÉTRICA E MODELAGEM DE TRANSISTORES BE (BACK ENHANCED) SOI MOSFET}

Dissertação apresentada à Escola
Politécnica da Universidade de São Paulo
como parte dos requisitos necessários para
a obtenção do título de Mestre em Ciências.

Área de Concentração:

Engenharia Elétrica / Microeletrônica.

Orientador: Prof. Dr. João Antonio Martino 
Este exemplar foi revisado e corrigido em relaçáo a versão original, sob responsabilidade unica do autor e com a anuéncia de seu orientador.

Sáo Paulo. 06 de $A B R: L$ de 2018

Assinatura do autor.

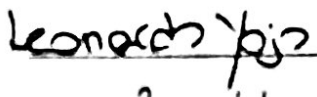

Assinatura do onentador porothichto

Catalogação-na-publicação

Yojo, Leonardo Shimizu

Estudo, Caracterização Elétrica e Modelagem de Transistores BE (Back Enhanced) SOI MOSFET / L. S. Yojo - versão corr. -- São Paulo, 2018. $99 \mathrm{p}$.

Dissertação (Mestrado) - Escola Politécnica da Universidade de São Paulo. Departamento de Engenharia de Sistemas Eletrónicos.

1.Microeletrônica 2.SOI MOSFET I.Universidade de São Paulo. Escola Politécnica. Departamento de Engenharia de Sistemas Eletrônicos II.t. 


\section{AGRADECIMENTOS}

Gostaria de agradecer ao Prof. Dr. João Antonio Martino pela orientação, pelos conhecimentos passados e pelo incentivo passados ao longo deste trabalho.

A todos os amigos do grupo SOI que sempre se mostraram dispostos a ajudar no decorrer do tempo. Gostaria de agradecer especialmente ao Rangel pela atenção e por todas as discussões que sempre contribuíram para melhorar o trabalho desde 0 início e à Kátia por todas as ajudas prestadas.

A meus familiares que me apoiaram e torceram por mim.

À FAPESP pelo apoio financeiro (projeto 2015/25100-3). 


\section{RESUMO}

Este trabalho tem como objetivo o estudo, caracterização elétrica e modelagem do novo transistor desenvolvido e fabricado no Laboratório de Sistemas Integráveis (LSI) da Universidade de São Paulo (USP) chamado BE (Back Enhanced) SOI MOSFET. Trata-se de um dispositivo inovador que se destaca principalmente pela sua facilidade de fabricação (exigindo apenas processos bem conhecidos e nenhuma etapa de dopagem do semicondutor) e sua flexibilidade quanto ao modo de operação (pode atuar como um transistor MOS tipo $n$ ou um transistor MOS tipo $p$, dependendo somente da polarização de substrato). Aplicando-se tensão no substrato ( $\left.V_{G B}\right)$ é possível formar um canal de elétrons $\left(V_{G B}>0\right)$ ou lacunas $\left(V_{G B}<0\right)$ na segunda interface da camada de silício, por onde a corrente entre fonte e dreno flui. Sua patente foi requerida junto ao INPI (Instituto Nacional da Propriedade Industrial) sob o número BR 102015020974 6. Foram realizadas medidas elétricas e simulações numéricas para melhor compreender seu princípio de funcionamento e as características que tornam possível sua reconfigurabilidade. Duas fabricações distintas deste tipo de dispositivo foram analisadas. Além das espessuras distintas, a principal diferença entre elas é o metal utilizado nos eletrodos de fonte e dreno, sendo alumínio na primeira e níquel na segunda versão. O alumínio utilizado na primeira versão resultou em contatos Ôhmicos após o processamento térmico das lâminas, que favoreceram o funcionamento do dispositivo como transistor tipo $\mathrm{p}$, devido à natureza do material utilizado. A análise em função da temperatura (de $25^{\circ} \mathrm{C}$ até $125^{\circ} \mathrm{C}$ ) mostrou uma variação da tensão de limiar (até $1,52 \mathrm{mV} /{ }^{\circ} \mathrm{C}$ ) e uma degradação da mobilidade dos portadores de carga (analisado através da transcondutância), resultando no surgimento de um ponto invariante com a temperatura, o chamado ZTC (Zero Temperature Coefficient). Já a segunda versão possui contatos Schottky, na qual foram obtidos níveis de corrente apreciáveis tanto para transistores tipo n (na ordem de $n A$ para as condições de polarização utilizadas), quanto para transistores tipo $p$ (na ordem de $\mu \mathrm{A}$ ). $\mathrm{O}$ comportamento da curva de corrente de dreno deste dispositivo apresentou uma estabilização a partir de determinado valor de tensão de porta. A partir deste ponto o BE SOI MOSFET deixa de atuar como um transistor convencional e passa a ter sua corrente de dreno proporcional a tensão de substrato. Medidas em função da temperatura nesta segunda versão permitiram comparar os resultados com os da primeira versão. Percebeu-se a ausência do ponto de ZTC, uma vez que foi 
observado o aumento da corrente devido à diminuição da resistência dos contatos de fonte e dreno para temperaturas mais elevadas. Por fim, a operação de um circuito inversor utilizando o BE SOI MOSFET foi implementada, mesmo quando alternando os tipos dos transistores, comprovando a flexibilidade de funcionamento dos transistores ao mudar seu tipo em função da polarização de substrato.

Palavras chaves: BE SOI MOSFET, tecnologia SOI, transistores reconfiguráveis. 


\section{ABSTRACT}

The aim of this work is the study, the electrical characterization and the modeling of the new transistor that was developed and fabricated in the Laboratório de Sistemas Integráveis (LSI) at University of Sao Paulo (USP). It was named BE (Back Enhanced) SOI MOSFET. This innovative device has the advantage of a simple fabrication (only well-known processes are required to build it and there is no need of any doping step) and it has a reconfigurable operation (it can act as a n-type MOS transistor or as a ptype MOS transistor depending only on substrate bias). The substrate voltage $\left(\mathrm{V}_{\mathrm{GB}}\right)$ is responsible for the formation of an electron $\left(\mathrm{V}_{\mathrm{GB}}>0\right)$ or a hole $\left(\mathrm{V}_{\mathrm{GB}}<0\right)$ channel at the back interface of the silicon, where the drain current flows. The patent for it was required at the National Industrial Property Institute under the number BR 102015 020974 6. Electrical measurements and numerical simulations were performed to better understand its functioning principle and the characteristics that enable its reconfigurability. Two different fabrication splits were analyzed. Beside their thicknesses, the main difference between them is the drain and source metal electrode (aluminum in the first split and nickel in the second one). The one with aluminum electrodes resulted in Ohmic contacts after thermal processing, that favored the formation on the p-type transistor because of the nature of the used element. It was observed a variation of the threshold voltage (up to $1.52 \mathrm{mV} /{ }^{\circ} \mathrm{C}$ ) and a mobility degradation (seen through the transconductance behavior) as a function of the temperature (from $25^{\circ} \mathrm{C}$ to $125^{\circ} \mathrm{C}$ ), resulting in a zero-temperature coefficient (ZTC) bias point in this device. In this bias condition point, the drain current is almost constant as a function of the temperature, which is a good characteristic especially for analog circuits. The second split has Schottky drain and source contacts, in which appreciable current levels were obtained for both $n$-type transistors (order of magnitude of $n A$ in the measured bias conditions) and p-type transistors (order of magnitude of $\mu \mathrm{A}$ ). The drain current of this device showed a particular behavior where the drain current stabilizes from a certain gate voltage. In this condition, the BE SOI MOSFET does not act as a conventional transistor anymore and its current is proportional to the substrate bias. Measurements as a function of the temperature were performed in the device too. It was observed an increase of the drain current, differently from the first split, due to the reduction of the source and drain contacts resistances as a function of the temperature. This resulted in the absence of the ZTC point. Finally, the operation of an 
inverter circuit using BE SOI MOSFET transistors was implemented, even if the type of the transistors were switched. This result shows the flexibility of operation of the transistor, in other words, it is possible to change its type as a function of the substrate bias.

Key words: BE SOI MOSFET, SOI technology, reconfigurable transistors. 


\section{LISTA DE FIGURAS}

Figura 1.1 - Evolução do número de transistores em circuitos integrados ao longo do tempo. 20

Figura 1.2 - Perfil de uma lâmina SOI. 21

Figura 2.1 - Perfil tridimensional de um transistor SOI MOSFET. .26

Figura 2.2 - Diagrama de bandas de energia em transistores SOI tipo n parcialmente depletado e completamente depletado. .27

Figura 2.3 - Modelo de capacitâncias entre porta e substrato. a) MOS convencional. b) FD SOI MOSFET.

Figura 2.4 - Exemplo de curvas de transferência de um transistor MOS tipo p que apresenta o ponto de ZTC 33

Figura 2.5 - Diagrama de bandas de energia para $E_{F M}>E_{F}$, formando uma junção Ôhmica entre metal e semicondutor, antes e depois do contato. .36

Figura 2.6 - Diagrama de bandas de energia para $E_{F M}<E_{F}$ e semicondutor fortemente dopado, se comportando como contato Ôhmico.

Figura 2.7 - Diagrama de bandas de energia para $E_{F M}<E_{F}$, formando um contato Schottky entre metal e semicondutor. 37

Figura 2.8 - Diagrama de bandas de energia para um contato Schottky diretamente polarizado $(A)$ e reversamente polarizado $(B)$ .38

Figura 2.9 - Circuito elétrico de um inversor CMOS. 39

Figura 2.10 - Modelo de chaves do inversor CMOS 39

Figura 2.11 - Curvas de carga para transistores nMOSFET e pMOSFET do circuito inversor CMOS.

Figura 2.12 - Característica de transferência de tensão do inversor CMOS com transistores casados.

Figura 3.1 - a) Perfil do BE SOI MOSFET após a primeira fotolitografia. b) Perfil do BE SOI MOSFET após a segunda fotolitografia. c) Perfil do BE SOI MOSFET após a terceira fotolitografia. 44

Figura 3.2 - Perfil esquemático do BE SOI MOSFET. 44

Figura 3.3 - Diagrama de bandas de energia esquemático mostrando o princípio de operação. a) BE SOI pMOSFET no estado ligado e a formação da camada de lacunas na segunda interface. b) BE SOI nMOSFET no estado ligado e a formação da camada de elétrons na segunda interface. c) BE SOI pMOSFET no 
estado cortado e a região depletada interrompendo a camada de lacunas na segunda interface. d) do BE SOI nMOSFET no estado cortado e a região depletada interrompendo a camada de elétrons na segunda interface.

Figura 3.4 - Leiaute do circuito integrado fabricado contendo diversos BE SOI MOSFETs. Em destaque os dispositivos utilizados. .48

Figura 3.5 - Seção transversal do BE SOI MOSFET simulado. 49

Figura 3.6 - Curvas de corrente de dreno em função da tensão de porta experimentais e simuladas. .50

Figura 3.7 - Exemplo de uma curva ID em função de $V_{G F}$ de um transistor tipo p....51 Figura 3.8 - Exemplo de uma curva $\mathrm{d}^{2} \mathrm{I}_{\mathrm{D}} / \mathrm{dV}_{\mathrm{GF}}{ }^{2}$ em função de $\mathrm{V}_{\mathrm{GF}}$ de um transistor tipo $p$, mostrando o método de extração da tensão de limiar.

Figura 3.9 - Exemplo de uma curva dl $/ \mathrm{dV}_{\mathrm{GF}}$ em função de $\mathrm{V}_{\mathrm{GF}}$ de um transistor tipo $p$, mostrando o método de extração da transcondutância máxima. 52

Figura 3.10 - Exemplo de uma curva $d V_{G F} / d\left(\log l_{D}\right)$ em função de $V_{G F}$ de um transistor tipo $p$, mostrando o método de extração da inclinação de sublimiar mínima. ....53 Figura 3.11 - Modelo de capacitâncias representando o acoplamento entre a porta e o substrato no BE SOI MOSFET. .54

Figura 4.1 - Perfil esquemático da primeira versão fabricada do BE SOI MOSFET. 55

Figura 4.2 - Foto da primeira versão do transistor BE SOI MOSFET fabricado. 55

Figura 4.3 - Curvas de transferência ID em função de $V_{G F}$ medidas experimentalmente para diferentes valores de $\mathrm{V}_{\mathrm{GB}}$. 56

Figura 4.4 - Curvas de transferência ID em função de $V_{G F}$ medidas experimentalmente para diferentes temperaturas em escala linear.

Figura 4.5 - Curvas de transferência ID em função de $V_{G F}$ medidas experimentalmente para diferentes temperaturas em escala logarítmica. .58

Figura 4.6 - Tensão de limiar em função da temperatura para diferentes valores de $\mathrm{V}_{\mathrm{GB}}$ .59

Figura 4.7 - Tensão de limiar em função da polarização $V_{G B}$ para diferentes temperaturas. .60

Figura 4.8 - Transcondutância máxima em função da temperatura para diferentes valores de $\mathrm{V}_{\mathrm{GB}}$.

Figura 4.9 - Inclinação de sublimiar em função da temperatura para diferentes valores de $V_{G B}$ 
Figura 4.10 - Comparação dos valores de $V_{Z T C}$ obtidos experimentalmente e através do modelo.

Figura 5.1 - Perfil esquemático da segunda versão fabricada do BE SOI MOSFET. 64

Figura 5.2 - Foto da primeira versão do transistor BE SOI MOSFET fabricado. .64

Figura 5.3 - Curvas de transferência lD em função de $V_{G F}$ medidas experimentalmente para diferentes valores de $\mathrm{V}_{\mathrm{GB}}$, mostrando o funcionamento do $\mathrm{BE} \mathrm{SOI}$ PMOSFET. 65

Figura 5.4 - Curvas de transferência ID em função de $V_{G F}$ medidas experimentalmente para diferentes valores de $\mathrm{V}_{\mathrm{GB}}$, mostrando o funcionamento do $\mathrm{BE}$ SOI nMOSFET .66

Figura 5.5 - Curva esquemática da corrente de dreno em função de $V_{\text {GF. }}$. .67

Figura 5.6 - Simulação numérica da densidade de cargas no corte transversal BB' da

Figura 3.2.

Figura 5.7 - Perfis esquemáticos do BE SOI MOSFET em diferentes regiões de operação. A) Operação como transistor em corte. B) Operação como transistor em condução. C) Operação não convencional. .70

Figura 5.8 - Curvas de transferência experimentais para diferentes valores de $V_{D S}$ do BE SOI PMOSFET. .71

Figura 5.9 - Tensão de limiar do BE SOI pMOSFET em função de $V_{D S}$. .72

Figura 5.10 - Curvas de transferência experimentais para diferentes valores de VDS do BE SOI nMOSFET .72

Figura 5.11 - Tensão de limiar do BE SOI nMOSFET em função de $V_{D S}$. .73

Figura 5.12 - Simulação numérica do diagrama de bandas de energia do BE SOI pMOSFET na segunda interface para $V_{D S}=-500 \mathrm{mV}$, $-300 \mathrm{mV}$ e $-100 \mathrm{mV}$ (corte $A A^{\prime}$ da Figura 3.2). .74

Figura 5.13 - Simulação numérica do diagrama de bandas de energia do BE SOI pMOSFET na segunda interface para $V_{D S}=100 \mathrm{mV}$ e $200 \mathrm{mV}$ (corte $A A^{\prime}$ ' da Figura 3.2). .74

Figura 5.14 - Simulação numérica do diagrama de bandas de energia do BE SOI nMOSFET na segunda interface para $V_{D S}=500 \mathrm{mV}, 300 \mathrm{mV}$ e $100 \mathrm{mV}$ (corte $A A^{\prime}$ da Figura 3.2)...... 
Figura 5.15 - Simulação numérica do diagrama de bandas de energia do BE SOI nMOSFET na segunda interface para $V_{D S}=-100 m V$ e $-200 m V$ (corte $A A^{\prime}$ da Figura 3.2). .76

Figura 5.16 - Curvas de corrente $\left|I_{D}\right|$ em função de $V_{G F}$ em função da temperatura para a segunda versão do BE SOI pMOSFET.

Figura 5.17 - Curvas de corrente ID em função de VGF em função da temperatura para a segunda versão do BE SOI nMOSFET.

Figura 5.18 - Simulação numérica da corrente de dreno para BE SOI pMOSFET com tsi $=23 \mathrm{~nm}$ e tox $=15 \mathrm{~nm}$ para diferentes temperaturas. A) Contatos ôhmicos. B) Contatos Schottky.

Figura 5.19 - Simulação numérica da corrente de dreno para BE SOI pMOSFET com tsi=10nm e tox=10nm para diferentes temperaturas. A) Contatos ôhmicos. B) Contatos Schottky.

Figura 5.20 - Potencial elétrico da simulação numérica do BE SOI pMOSFET com tsi=23nm e tox=15nm ao longo da segunda interface para diferentes temperaturas.

A) Contatos ôhmicos. B) Contatos Schottky. 80

Figura 5.21 - Potencial elétrico da simulação numérica do BE SOI pMOSFET com $\mathrm{t} s \mathrm{~s}=10 \mathrm{~nm}$ e tox $=10 \mathrm{~nm}$ ao longo da segunda interface para diferentes temperaturas. A) Contatos ôhmicos. B) Contatos Schottky. 80

Figura 5.22 - Densidade de corrente de lacunas simulada no corte transversal do canal para o transistor BE SOI pMOSFET com tsi=23nm.

Figura 5.23 - Circuito inversor utilizando o BE SOI MOSFET e a curva de transferência do inversor medida experimentalmente.

Figura 5.24 - Curva de transferência do inversor alternando a polarização do circuito.

Figura 5.25 - Tensão de limiar em função de $\mathrm{V}_{\mathrm{GB}}$. 85

Figura 5.26 - Tensão de limiar simulada do BE SOI MOSFET com tox fixo e tsi variando.

Figura 5.27 - Tensão de limiar simulada do BE SOI MOSFET com tox variando e tsi fixo. 


\section{LISTA DE SIGLAS E ABREVIATURAS}

BE Back Enhanced (Enriquecido pelo substrato)

CMOS Complementary Metal-Oxide-Semiconductor (Metal Óxido Semicondutor Complementar)

FD Fully Depleted (Totalmente depletado)

finFET Fin Field-Effect-Transistor (Transistor de efeito de campo com aleta) INPI Instituto Nacional da Propriedade Industrial Laboratório de Sistemas Integráveis

MOSFET Metal-Oxide-Semiconductor Field-Effect Transistor (Transistor de efeito de campo metal óxido semicondutor)

nMOSFET n-channel Metal-Oxide-Semiconductor Field-Effect Transistor (Transistor de efeito de campo metal óxido semicondutor com canal tipo $n$ )

PADIS Programa de Apoio ao Desenvolvimento Tecnológico da Indústria de Semicondutores e Displays

PD Partially Depleted (Parcialmente depletado)

pMOSFET p-channel Metal-Oxide-Semiconductor Field-Effect Transistor (Transistor de efeito de campo metal óxido semicondutor com canal tipo p)

SCE Short Channel Effect (Efeito de canal curto)

SIMOX Separation by Implanted Oxygen (Técnica de fabricação de lâmina SOI por implantação de oxigênio)

SOI Silicon-On-Insulator (Silício sobre isolante)

USP Universidade de São Paulo

UTBB Ultra Thin Body and Burried Oxide (Corpo e óxido enterrado ultra finos)

ZTC Zero Temperature Coefficient (Ponto invariante com a temperatura) 


\section{LISTA DE SÍMBOLOS}

$\Delta T$

$\Delta \mathrm{V}_{\mathrm{T}}$

C

$\mathrm{C}_{\text {BOX }}$

$\mathrm{C}_{D}$

Cox1

$\mathrm{C}_{\mathrm{Si}}$

CT

$E_{c}$

$E_{F}$

$E_{F M}$

$E_{g}$

$E_{i}$

$E_{v}$

gm

gmmáx

$\mathrm{gm}_{\text {sat }}$

gmtriodo

GND

$h_{d}$

$h_{s}$

ID

IDn

$\mathrm{IDp}$

Variação de temperatura $\left[{ }^{\circ} \mathrm{C}\right]$

Variação de tensão de limiar [V]

Fator de degradação da mobilidade com a temperatura

Capacitância do óxido enterrado por unidade de área [F/cm²]

Capacitância da região de depleção por unidade de área [F/cm²]

Capacitância do óxido de porta por unidade de área [F/cm²]

Capacitância da camada de silício por unidade de área [F/cm²]

Fator de degradação da mobilidade com a temperatura para a temperatura $\mathrm{T}$.

Nível energético mínimo da banda de condução [eV]

Nível de Fermi do semicondutor [eV]

Nível de Fermi do metal $[\mathrm{eV}]$

Banda proibida [eV]

Nível intrínseco do semicondutor [eV]

Nível energético máximo da banda de valência [eV]

Transcondutância [S]

Transcondutância máxima [S]

Transcondutância do transistor na região de saturação [S]

Transcondutância do transistor na região de triodo [S]

Tensão de referencia [V]

Altura da barreira de potencial entre dreno e porta [V]

Altura da barreira de potencial entre fonte e porta [V]

Corrente de dreno $[\mathrm{A}]$

Corrente de dreno do transistor tipo $\mathrm{n}[\mathrm{A}]$

Corrente de dreno do transistor tipo $p[A]$ 


\begin{tabular}{|c|c|}
\hline $\mathrm{k}$ & Constante de Boltzmann $[\mathrm{eV} / \mathrm{K}]$ \\
\hline L & Comprimento do canal $[\mu \mathrm{m}]$ \\
\hline $\mathrm{n}$ & Fator de corpo \\
\hline $\mathrm{Na}_{\mathrm{a}}$ & Concentração de dopantes aceitadores $\left[\mathrm{cm}^{-3}\right]$ \\
\hline $\mathrm{n}_{\mathrm{i}}$ & Concentração intrínseca de portadores $\left[\mathrm{cm}^{-3}\right]$ \\
\hline q & Carga elementar do elétron $[\mathrm{C}]$ \\
\hline Qdepl & $\begin{array}{l}\text { Densidade de carga total em depleção da camada de silício por } \\
\text { unidade de área }\left[\mathrm{C} / \mathrm{cm}^{2}\right]\end{array}$ \\
\hline Qinv1 & $\begin{array}{l}\text { Densidade de cargas invertidas no canal próximo da primeira } \\
\text { interface por unidade de área }\left[\mathrm{C} / \mathrm{cm}^{2}\right]\end{array}$ \\
\hline Qox1 & $\begin{array}{l}\text { Densidade de cargas fixas na primeira interface por unidade de } \\
\text { área }\left[\mathrm{C} / \mathrm{cm}^{2}\right]\end{array}$ \\
\hline Qox2 & $\begin{array}{l}\text { Densidade de cargas fixas na segunda interface por unidade de } \\
\text { área }\left[\mathrm{C} / \mathrm{cm}^{2}\right]\end{array}$ \\
\hline Qs2 & $\begin{array}{l}\text { Densidade de cargas no canal na segunda interface por unidade de } \\
\text { área }\left[\mathrm{C} / \mathrm{cm}^{2}\right]\end{array}$ \\
\hline $\mathrm{S}$ & Inclinação de sublimiar [mV/dec.] \\
\hline $\mathrm{T}$ & Temperatura $[\mathrm{K}]$ \\
\hline tbox & Espessura do óxido enterrado [nm] \\
\hline tox & Espessura do óxido de porta [nm] \\
\hline$t_{s i}$ & Espessura do silício [nm] \\
\hline $\mathrm{V}_{\mathrm{a}}$ & Tensão aplicada à junção metal semicondutor [V] \\
\hline$V_{D D}$ & Tensão de alimentação do circuito [V] \\
\hline$V_{D S}$ & Tensão entre fonte e dreno [V] \\
\hline$V_{D S n}$ & Tensão entre fonte e dreno do transistor tipo $\mathrm{n}[\mathrm{V}]$ \\
\hline$V_{\text {DSp }}$ & Tensão entre fonte e dreno do transistor tipo $p$ [V] \\
\hline $\mathrm{V}_{\mathrm{FB} 1}$ & Tensão de faixa plana da porta [V] \\
\hline
\end{tabular}




\begin{tabular}{|c|c|}
\hline$V_{G B}$ & Tensão de substrato [V] \\
\hline$V_{G B, a c 2}$ & Tensão de substrato para a segunda interface em acumulação [V] \\
\hline$V_{G F}$ & Tensão de porta [V] \\
\hline$V_{G F n}$ ou $V_{G S n}$ & Tensão de porta do transistor tipo $\mathrm{n}[\mathrm{V}]$ \\
\hline$V_{G F p}$ ou $V_{G S p}$ & Tensão de porta do transistor tipo $p[\mathrm{~V}]$ \\
\hline$V_{i}$ & $\begin{array}{l}\text { Barreira de potencial devido ao encurvamento das bandas de } \\
\text { energia }[\mathrm{eV}]\end{array}$ \\
\hline$V_{\text {in }}$ & Tensão de entrada do circuito [V] \\
\hline$V_{\text {out }}$ & Tensão de saída do circuito [V] \\
\hline $\mathrm{V}_{\mathrm{T}}$ & Tensão de limiar [V] \\
\hline $\mathrm{V}_{\mathrm{T}, \mathrm{ac} 2}$ & Tensão de limiar para a segunda interface em acumulação [V] \\
\hline $\mathrm{V}_{\mathrm{T}, \mathrm{dep} 2}$ & Tensão de limiar para a segunda interface em depleção [V] \\
\hline $\mathrm{V}_{\mathrm{T}, \text { inv2 }}$ & Tensão de limiar para a segunda interface invertida [V] \\
\hline $\mathrm{V}_{\mathrm{Tn}}$ & Tensão de limiar do transistor $n$ [V] \\
\hline$V_{T p}$ & Tensão de limiar do transistor $\mathrm{p}[\mathrm{V}]$ \\
\hline VZTC & Tensão na porta do transistor no ponto de ZTC [V] \\
\hline W & Largura do canal $[\mu \mathrm{m}]$ \\
\hline$x d$ & Profundidade da região de depleção $[\mu \mathrm{m}]$ \\
\hline Xdmáx & Profundidade da região de depleção máxima $[\mu \mathrm{m}]$ \\
\hline$\alpha$ & Razão entre as capacitâncias vista acima e abaixo do canal \\
\hline aconv & $\begin{array}{l}\text { Razão entre as capacitâncias vista acima e abaixo do canal em } \\
\text { transistores de corpo MOSFET }\end{array}$ \\
\hline$\alpha_{\mathrm{FDSOI}}$ & $\begin{array}{l}\text { Razão entre as capacitâncias vista acima e abaixo do canal em } \\
\text { transistores FDSOI MOSFET }\end{array}$ \\
\hline$\alpha_{\text {FDSOlacc }}$ & $\begin{array}{l}\text { Razão entre as capacitâncias vista acima e abaixo do canal em } \\
\text { transistores FDSOI MOSFET com a segunda interface acumulada }\end{array}$ \\
\hline ox & Permissividade do óxido [F/cm] \\
\hline
\end{tabular}




\begin{tabular}{|c|c|}
\hline$\varepsilon \mathrm{Si}$ & Permissividade do silício [F/cm] \\
\hline$\theta$ & $\begin{array}{l}\text { Fator de degradação da mobilidade por campo elétrico transversal } \\
{[1 / \mathrm{V}]}\end{array}$ \\
\hline$\mu$ & Mobilidade dos portadores de carga móveis [ $\left.\mathrm{cm}^{2} / \mathrm{Vs}\right]$ \\
\hline$\mu_{0}$ & $\begin{array}{l}\text { Mobilidade dos portadores de carga móveis de baixo campo } \\
{\left[\mathrm{cm}^{2} / \mathrm{Vs}\right]}\end{array}$ \\
\hline Mef & Mobilidade dos portadores de carga móveis efetiva[cm²/Vs] \\
\hline$\phi b$ & Barreira de potencial $[\mathrm{eV}]$ \\
\hline$\phi F$ & Potencial de Fermi [eV] \\
\hline$\Phi_{\mathrm{m}}$ & Função trabalho do metal [eV] \\
\hline$\Phi_{\mathrm{MS1}}$ & $\begin{array}{l}\text { Diferença da função trabalho entre o metal e o semicondutor na } \\
\text { primeira interface }[\mathrm{eV}]\end{array}$ \\
\hline$\Phi_{\mathrm{MS} 2}$ & $\begin{array}{l}\text { Diferença da função trabalho entre o metal e o semicondutor na } \\
\text { segunda interface }[\mathrm{eV}]\end{array}$ \\
\hline$\phi s 1$ & Potencial de superfície na primeira interface [eV] \\
\hline$\phi s 2$ & Potencial de superfície na segunda interface [eV] \\
\hline$X$ & Afinidade elétrica do semicondutor [eV] \\
\hline
\end{tabular}




\section{SUMÁRIO}

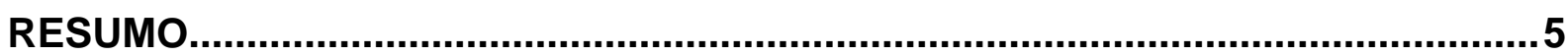

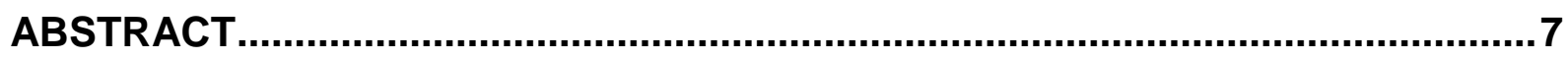

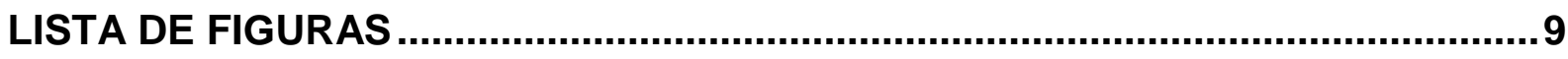

LISTA DE SIGLAS E ABREVIATURAS ...................................................... 13

LISTA DE SÍMBOLOS ...................................................................................14

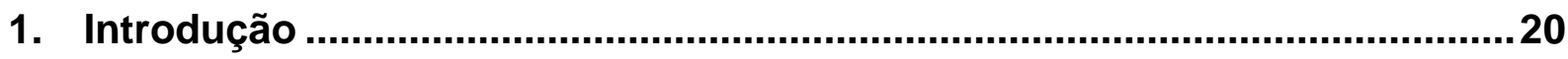

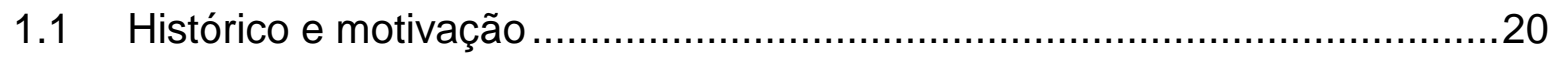

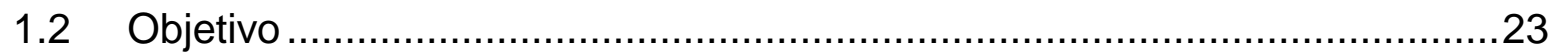

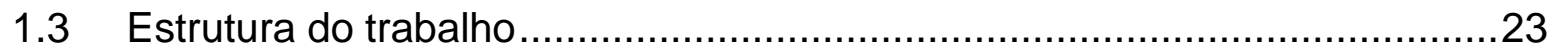

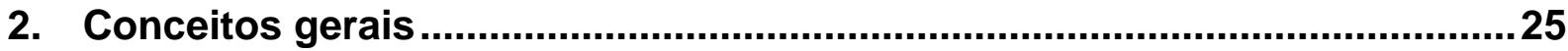

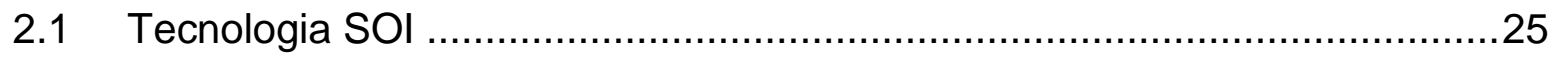

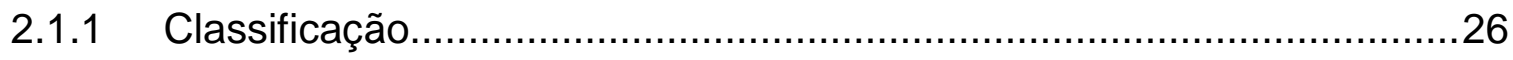

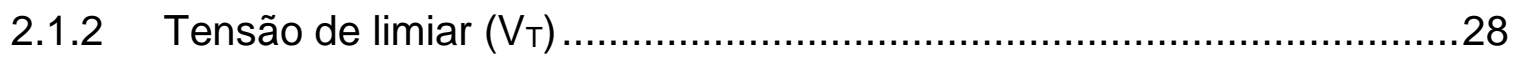

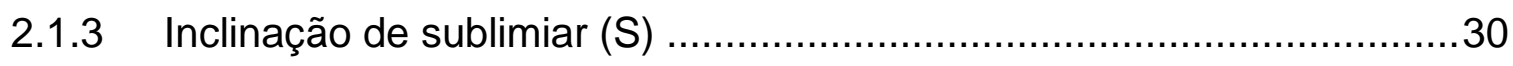

2.1.4 Efeito de corpo na tensão de limiar ..........................................32

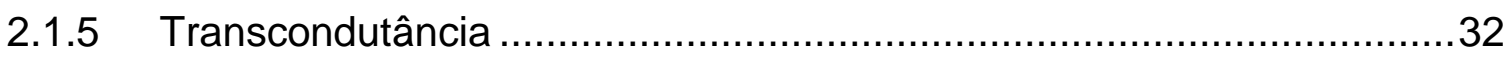

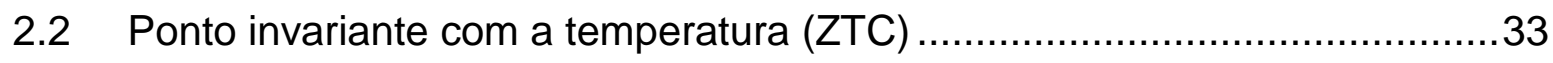

2.2.1 Modelo para VzTc na região linear ................................................ 34

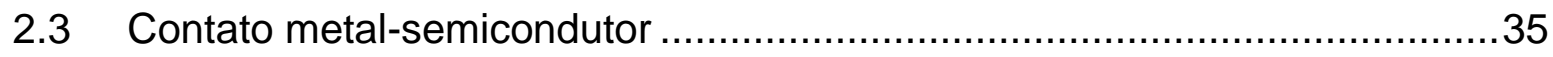

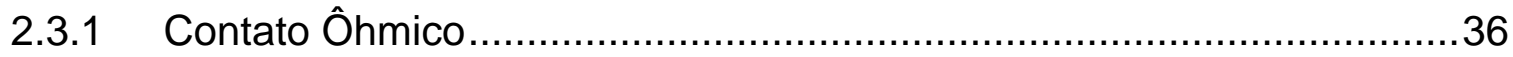

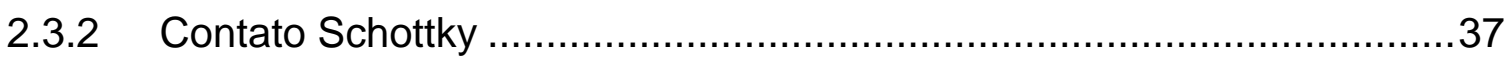

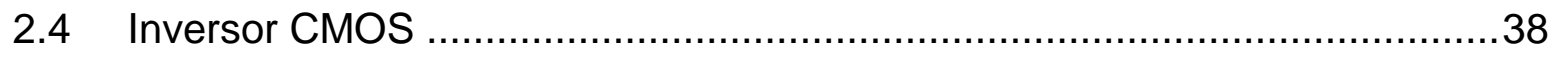

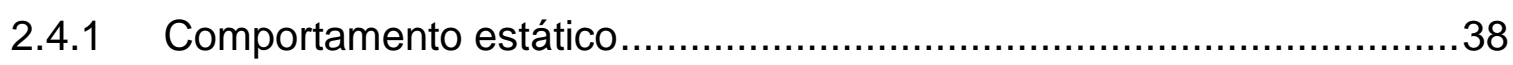

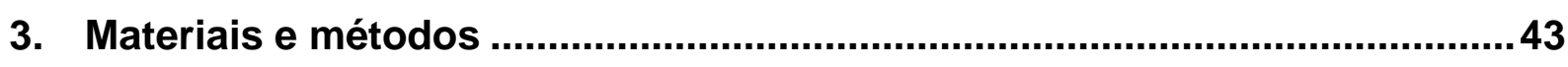

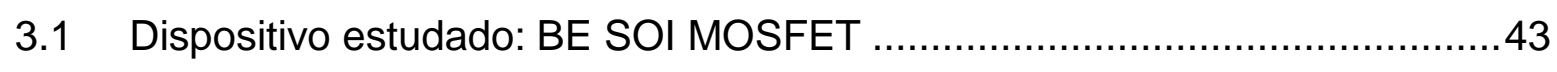

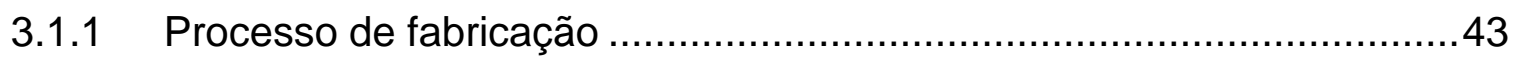




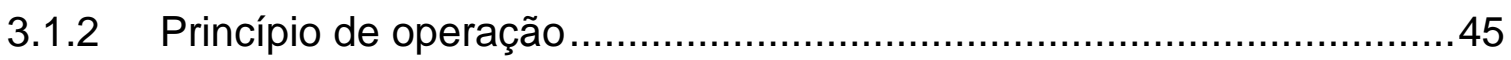

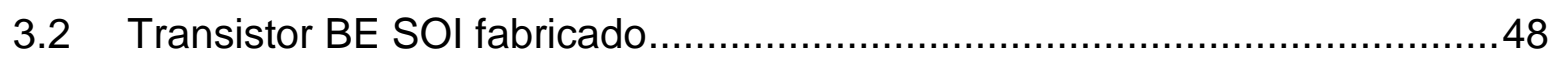

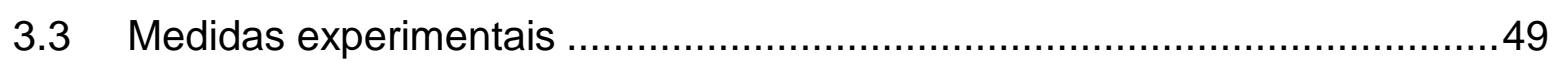

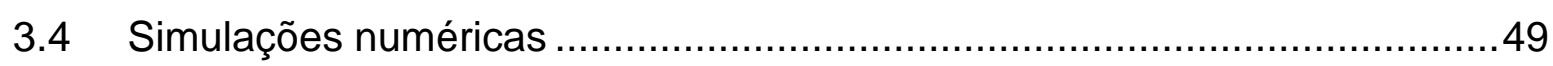

3.5 Extração dos parâmetros ..................................................................

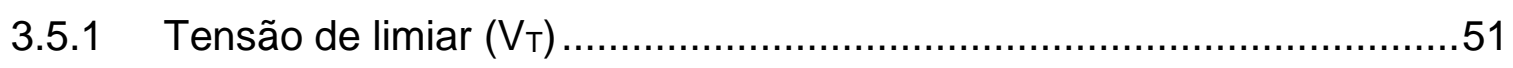

3.5.2 Transcondutância máxima (gmmáx) ……………………………....52

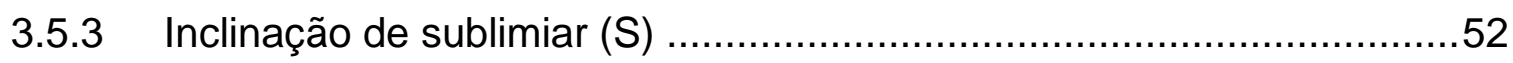

3.5.4 Fator de degradação da mobilidade com a temperatura (c) ..................53

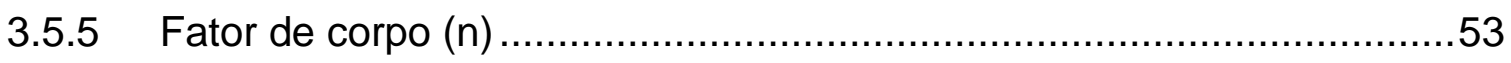

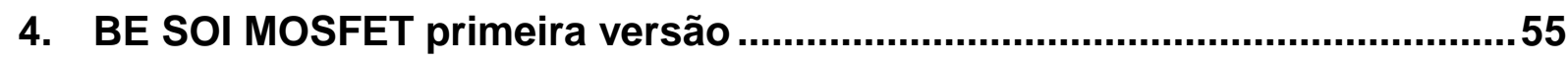

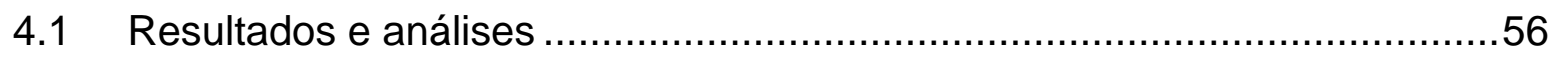

4.1.1 Medidas em temperatura ambiente .................................................56

4.1.2 Medidas em altas temperaturas e ZTC ............................................57

4.1.3 Determinação do ponto invariante com a temperatura ZTC na região

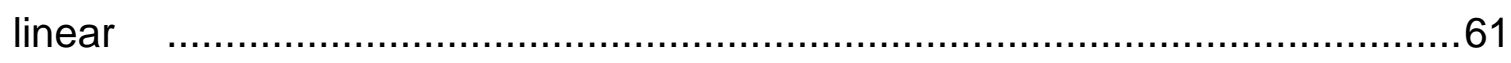

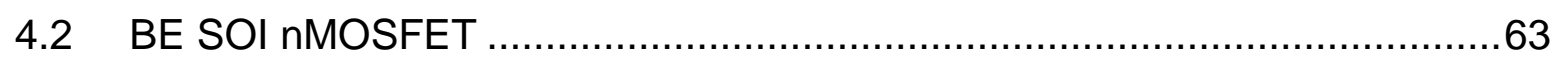

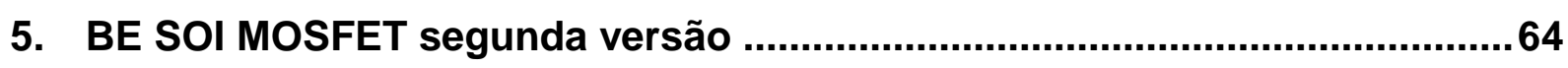

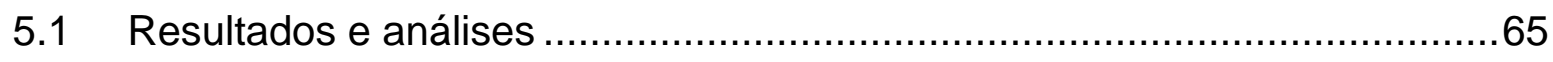

5.1.1 Medidas em função da polarização VDS ……………..........................70

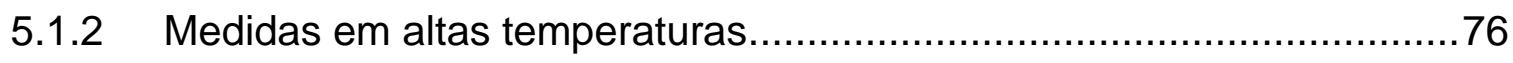

5.1.3 Comparação com a primeira versão do BE SOI ....................................78

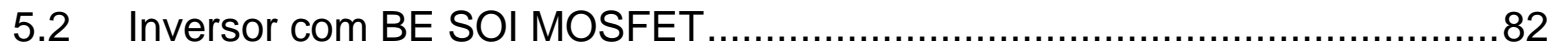

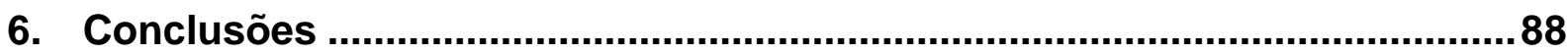

PUBLICAÇÕES GERADAS DURANTE O MESTRADO ........................................ 91

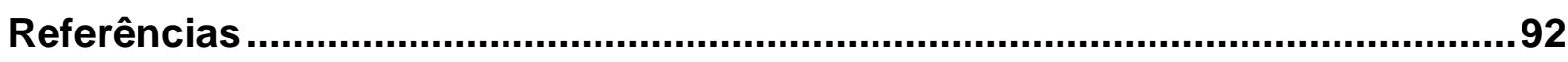

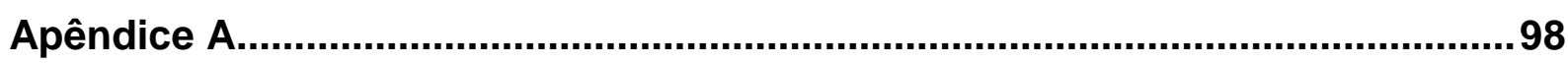




\section{Introdução}

\subsection{Histórico e motivação}

Atualmente a microeletrônica está amplamente difundida nas sociedades, em diversas aplicações e em todos os setores da economia, seja nos lares, nas indústrias, nos comércios ou nos campos. Equipamentos como celulares, computadores, automóveis, máquinas industriais, aviões, etc. dependem muito dos avanços tecnológicos nesta área do conhecimento, que se tornou fundamental para o avanço da humanidade e está presente no dia-a-dia de todos.

O primeiro circuito integrado criado em 1959 por Jack Kilby [1] e o surgimento da primeira versão prática do transistor MOSFET (Metal-Oxide-Semiconductor Field Effect Transistor) em 1960 [2] foram marcos importantes para a história da microeletrônica [3]. A partir desta data, essas tecnologias foram amplamente utilizadas e representam grande parcela dos circuitos eletrônicos produzidos mundialmente.

O transistor MOSFET possui uma estrutura composta de metal, óxido e semicondutor e funciona pelo controle do fluxo de corrente a partir de um campo elétrico. Já o circuito integrado possibilitou a criação de circuitos elétricos em escala industrial com melhores desempenhos e menores custos por transistor.

Figura 1.1 - Evolução do número de transistores em circuitos integrados ao longo do tempo.

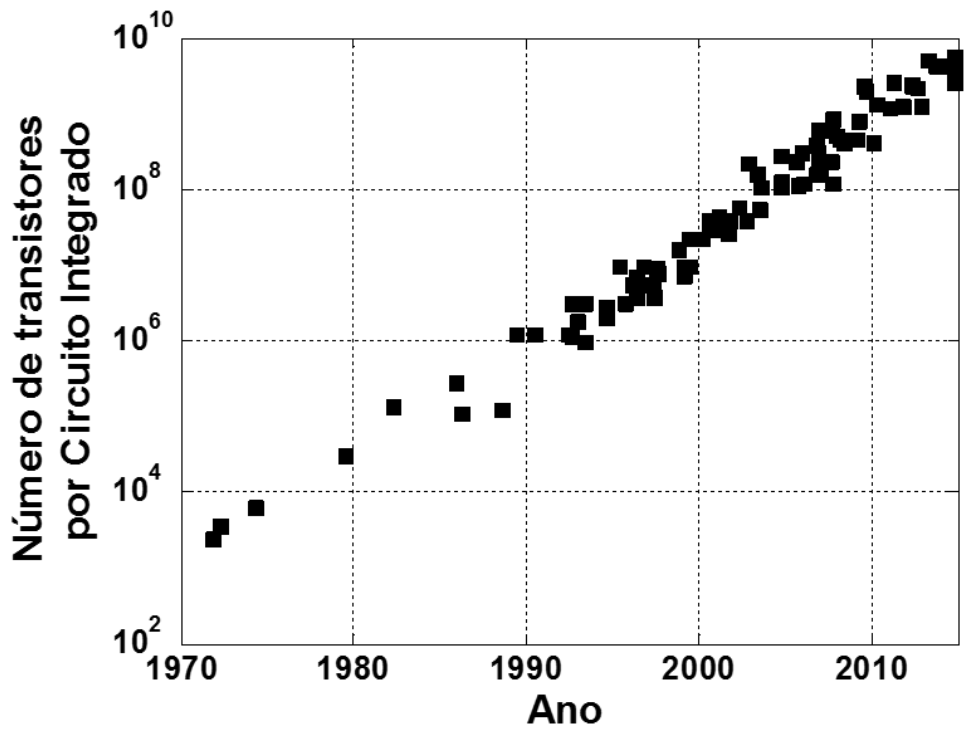

Fonte: Adaptado de [4] 
Com o desenvolvimento dos processos de fabricação, foi possível reduzir as dimensões dos transistores. A diminuição do tamanho possibilitou o aumento da integração, circuitos elétricos mais rápidos e menor consumo de potência.

Gordon Moore publicou em 1965 seu trabalho sobre a evolução dos circuitos integrados, no qual consta sua observação sobre a tendência do número de transistores num mesmo circuito integrado dobrar a cada 12 meses [5], que ficou conhecida como Lei de Moore. Essa tendência se mostrou válida com 0 desenvolvimento da indústria de semicondutores, diminuindo um pouco a taxa de variação para 18 e 24 meses mais recentemente. A Figura 1.1 mostra o crescimento exponencial do número de transistores em um circuito integrado, ao longo do tempo.

A partir de determinado ponto, o avanço da tecnologia dos transistores começou a ser afetado por efeitos parasitários indesejados que surgiram devido a miniaturização de suas dimensões. Efeitos como SCE (Short Channel Effects), disparo do tiristor parasitário e aumento das capacitâncias de junções prejudicavam seu desempenho.

Para contornar esses efeitos parasitários, novas tecnologias surgiram. Uma delas é o Silício-Sobre-Isolante (SOI - Silicon-On-Insulator) [6] [7] [8] [9]. Nela, os transistores são fabricados em uma camada de silício que está isolada do substrato, conforme mostrado na Figura 1.2. Dentre os benefícios podemos citar a supressão do disparo do tiristor parasitário, menores correntes de fuga e menores variações da tensão de limiar [10] [11]. Tais características também fazem da tecnologia SOI uma forte candidata para aplicações em altas temperaturas [12].

Figura 1.2 - Perfil de uma lâmina SOI.

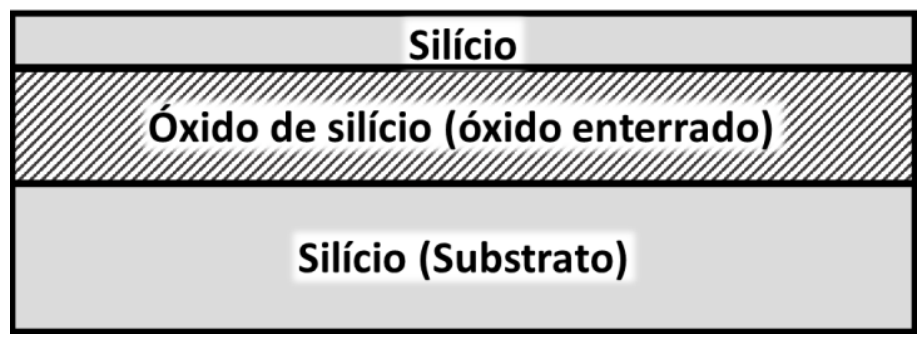

Fonte: Autor.

Diversas aplicações necessitam de circuitos integrados capazes de operar corretamente sob ambientes severos. Em especial, aplicações como em automobilística, aeronáutica e determinadas indústrias precisam de circuitos 
integrados operando em altas temperaturas [13] [14]. Nesse contexto, uma alternativa é polarizar os circuitos integrados em um ponto em que suas características não variem com a temperatura. Essa condição de polarização já vem sido estudada [15] [16] e é conhecida como ponto de ZTC (Zero Temperature Coefficient).

A performance dos transistores continuou avançando com novas arquiteturas que procuravam melhorar o controle eletrostático do canal, como por exemplo transistores UTBB (Ultra Thin Body and Burried Oxide) SOI [17], transistores tipo finFET [18] e transistores tipo nanofio [19]. Dessa forma foi possível reduzir os efeitos de canal curto, o que permite criar circuitos que ocupam menor área do circuito integrado.

Uma outra perspectiva para economizar área do circuito integrado é utilizar uma mesma região para implementar mais de uma função. Esse conceito é possível por meio de transistores reconfiguráveis [20] [21] [22] [23] [24], ou seja, que podem atuar como transistores tipo $\mathrm{n}$ ou tipo $\mathrm{p}$. Trabalhos recentes mostram circuitos de transistores reconfiguráveis operando duas funções lógicas distintas na tecnologia CMOS [25] [26] [27] [28].

No Brasil, a indústria de semicondutores está em seus estágios iniciais de desenvolvimento, existindo inclusive um Programa de Apoio ao Desenvolvimento Tecnológico da Indústria de Semicondutores e Displays (PADIS) [29], que inclui benefícios fiscais com o objetivo de atrair investimentos nas áreas de semicondutores e insumos estratégicos para cadeia produtiva. O fato de não possuir os produtores de insumos estratégicos e tão pouco os fabricantes dos sofisticados equipamentos necessários à complexa cadeia produtiva tornam mais difíceis as iniciativas nesta indústria, em nosso país.

Neste contexto, foi desenvolvido recentemente um processo de fabricação de um tipo de transistores MOSFET, cuja patente foi requerida junto ao INPI [30], que simplifica significativamente a fabricação de transistores MOSFET, o chamado BE (Back Enhanced) SOI MOSFET. Outra característica marcante deste dispositivo é sua flexibilidade quanto ao tipo de transistor, podendo atuar como um nMOSFET ou como um pMOSFET.

O alvo deste trabalho é o estudo do novo transistor BE SOI MOSFET [31], desenvolvido e fabricado no Laboratório de Sistemas Integráveis (LSI) da 
Universidade de São Paulo (USP). Trata-se de uma arquitetura inovadora desenvolvida a partir das ideias de dispositivos sem junção como o "Gate Resistors" [32] e transistores do tipo nanofio [33] sem dopagem e da técnica de caracterização de lâminas de silício $\psi$-MOSFET [34].

\subsection{Objetivo}

O objetivo deste trabalho é estudar o BE (Back Enhanced) SOI MOSFET para melhor compreender seu princípio de operação a partir de dispositivos já fabricados. Como se trata de um dispositivo novo, será realizado o estudo dos principais parâmetros elétricos, explorando uma de suas principais características, a reconfigurabilidade. Sua operação tanto como transistor tipo n ou como transistor tipo p será analisado por meio de medidas elétricas e simulações numéricas. Além disso, será levantado seu comportamento em altas temperaturas (até $150^{\circ} \mathrm{C}$ ). Um circuito inversor será analisado, alternando o tipo dos transistores para comprovar sua flexibilidade quanto ao modo de operação.

\subsection{Estrutura do trabalho}

Este trabalho está dividido em seis capítulos:

Capítulo 1 - Introdução: esse capítulo aborda a evolução da microeletrônica de forma breve, citando a Lei de Moore como um guia para esse avanço. Além disso, são levantados aspectos relevantes para o trabalho como a importância das aplicações em altas temperaturas e o conceito de transistores reconfiguráveis.

Capítulo 2 - Conceitos gerais: os principais conceitos abordados ao longo do trabalho são descritos nesse capítulo. Entre os temas mencionados estão a tecnologia SOI e alguns parâmetros elétricos, o conceito de ZTC, a diferenciação dos tipos de contato entre metal e semicondutor e o inversor CMOS.

Capítulo 3 - Materiais e métodos: este capítulo descreve o transistor estudado, suas principais características e seu princípio de funcionamento. Também são abordados os métodos de extração dos parâmetros utilizados para o estudo, a partir das medidas elétricas realizadas e as simulações numéricas do dispositivo. 
Capítulo 4 - BE SOI MOSFET primeira versão: o primeiro BE SOI MOSFET fabricado é explorado neste capítulo. Os primeiros resultados são mostrados e em seguida foi realizado um estudo de seu comportamento para temperaturas elevadas.

Capítulo 5 - BE SOI MOSFET segunda versão: esse capítulo descreve as diferenças entre a segunda versão do BE SOI MOSFET e sua primeira versão. A característica de transistor reconfigurável é atingida nesta versão. Além disso, será analisado uma condição de polarização entre fonte e dreno não convencional que se mostrou possível neste dispositivo. Também será realizada a comparação com a primeira versão em relação a presença do ponto de ZTC. Por fim, para comprovar a reconfigurabilidade do transitor, será construído e medido um circuito inversor.

Capítulo 6 - Conclusões parciais e sequência do trabalho: as conclusões realizadas a partir dos estudos dos capítulos anteriores são elencadas neste capítulo. Também estão incluídos os tópicos que serão abordados na sequência do trabalho. 


\section{Conceitos gerais}

Neste capítulo serão abordados os principais conceitos básicos que serão utilizados ao longo do trabalho.

\subsection{Tecnologia SOI}

A tecnologia de transistores SOI MOSFET é uma evolução da tecnologia MOS FET (Metal-Óxido-Semicondutor Field-Effect-Transistor). Seu princípio de funcionamento baseia-se no controle das cargas no canal, induzidas abaixo do isolante de porta, na estrutura do capacitor de porta formado pelo metal, óxido e semicondutor (MOS). Detalhes sobre o funcionamento deste tipo de dispositivo podem ser encontrados em [35].

Transistores SOI (Silicon-On-Insulator) são fabricados utilizando lâminas que possuem uma camada de óxido isolante, que separa a região ativa do circuito do substrato. Esta é a principal diferença entre o SOI MOSFET e o MOSFET convencional. Essa camada isolante pode propiciar diversos benefícios tais como a eliminação do efeito tiristor parasitário, redução de capacitâncias parasitárias, eliminação do risco de perfuração de junções rasas, maior robustez quanto ao efeito transitório da radiação, operação em altas temperaturas, etc. [10].

Uma lâmina SOI pode ser produzida por diversos métodos distintos. Uma técnica utilizada é o chamado SIMOX [10], no qual uma dose elevada de íons de oxigênio é implantada com alta energia em uma lâmina de silício. Após uma etapa de recozimento ocorre a formação do óxido enterrado. Devido ao processo de implantação iônica, esta camada de óxido possui distribuição gaussiana, e, portanto, a interface silício/óxido não é abrupta. Outra consequência é o elevado número de defeitos devido à implantação.

Outra opção para a fabricação de lâminas SOI é o chamado Smart-Cut [10]. Primeiramente é realizado a implantação iônica de hidrogênio em uma lâmina de silício previamente oxidada. Em seguida ocorre a união desta lâmina com uma outra de silício previamente oxidada. Após tratamento térmico apropriado ocorre uma separação desta lâmina na região onde se concentram os íons de hidrogênio 
implantados. Depois de uma etapa de polimento obtém-se uma lâmina SOI com melhor qualidade e espessuras de óxido enterrado mais finas que a técnica anterior.

Figura 2.1 - Perfil tridimensional de um transistor SOI MOSFET.

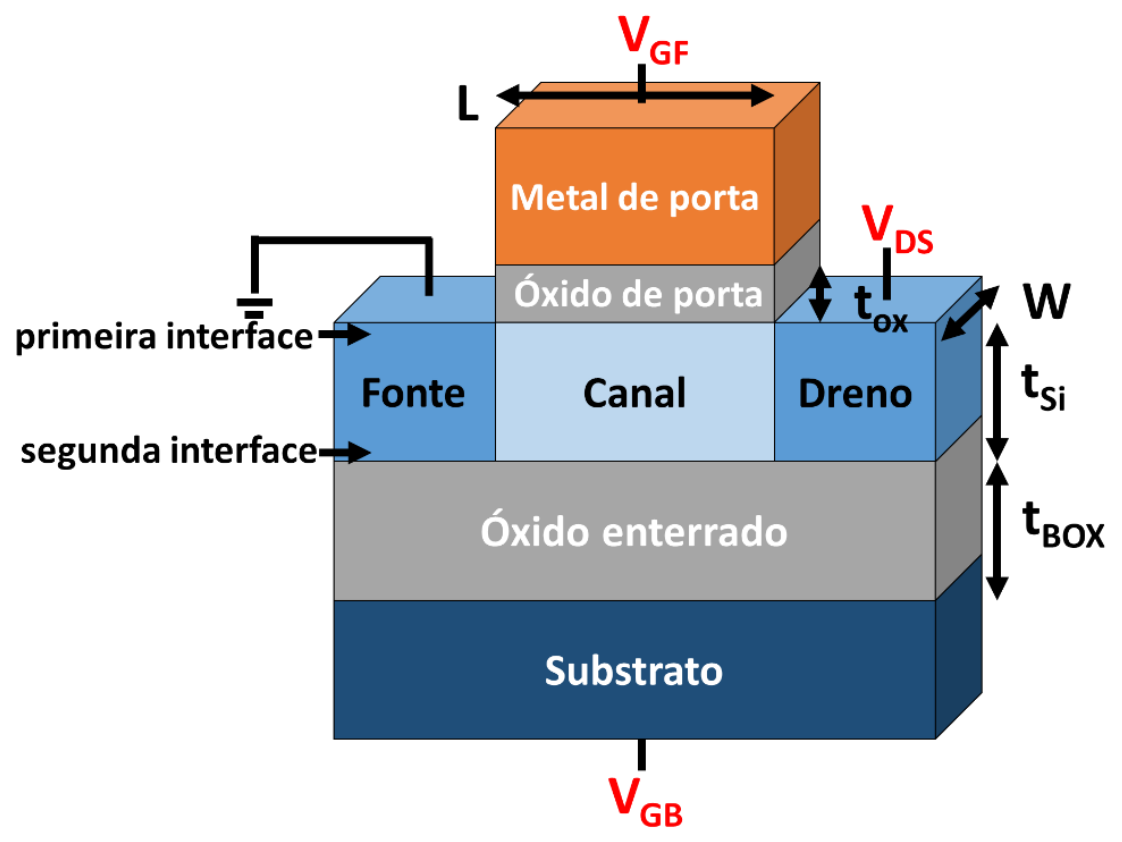

Fonte: Autor.

A Figura 2.1 mostra o perfil tridimensional esquemático de um transistor SOI MOSFET. O comprimento do transistor é $L$, a largura é $W$, a espessura do óxido de porta é tox, a espessura do silício é tsi e a espessura do óxido enterrado isolante é tBox.

\subsubsection{Classificação}

As características e propriedades dos transistores SOI MOSFETs são muito dependentes da espessura ( $\mathrm{ts}_{\mathrm{i}}$ ) e da concentração de dopantes da camada de silício. Há dois tipos de transistores SOI: parcialmente depletado (PD - Partially Depleted SOI) e totalmente depletado (FD - Fully Depleted SOI). 
Figura 2.2 - Diagrama de bandas de energia em transistores SOI tipo n parcialmente depletado e completamente depletado.
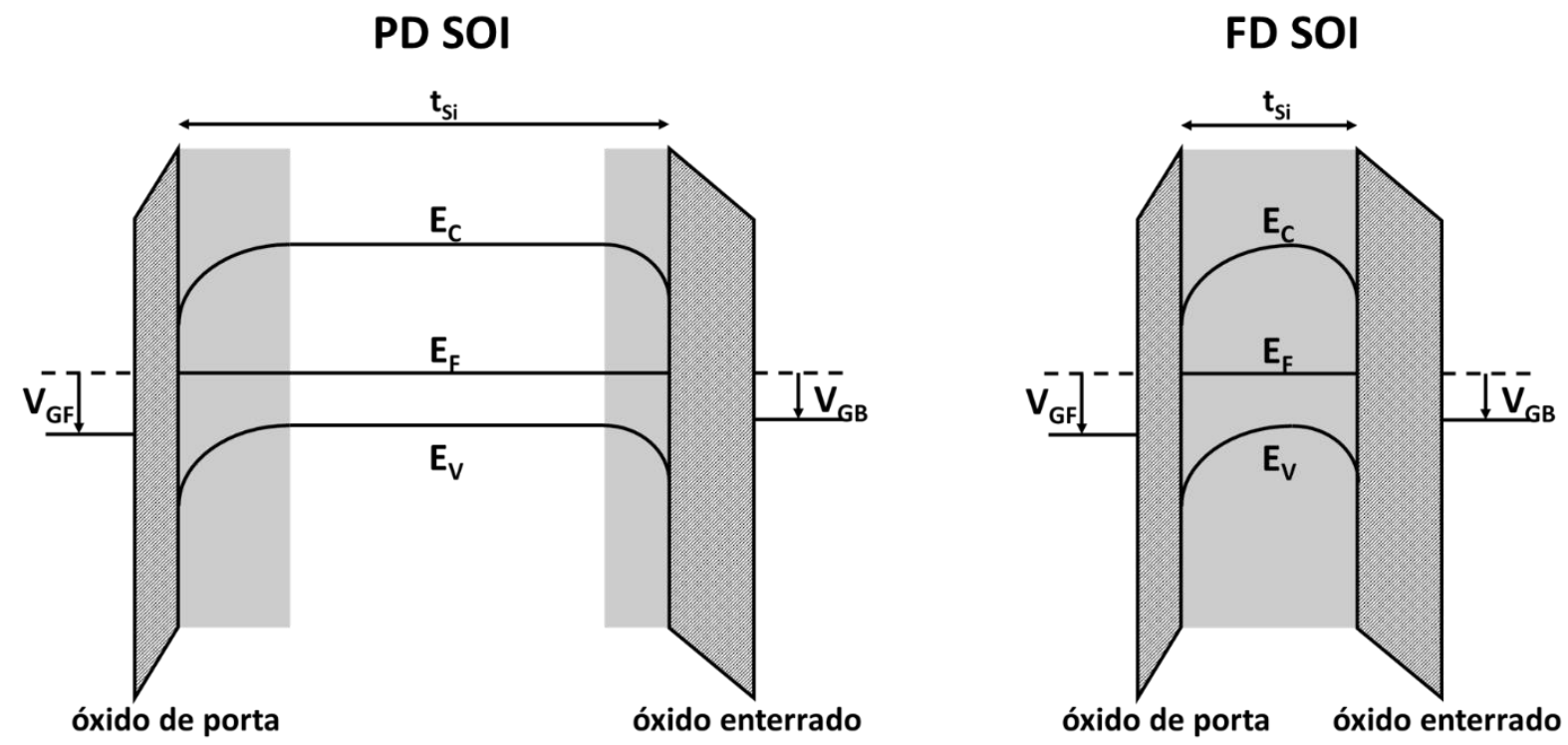

Fonte: autor.

A Figura 2.2 mostra o diagrama de bandas para o transistor parcialmente depletado (a esquerda) e completamente depletado (a direita), representados na situação em que a tensão de porta ( $\left.\mathrm{V}_{\mathrm{GF}}\right)$ equivale a tensão de limiar $\left(\mathrm{V}_{\mathrm{T}}\right)$. As regiões sombreadas representam o silício depletado. A tensão aplicada ao eletrodo de substrato é $V_{\mathrm{GB}}$.

O valor da largura máxima que a região pode atingir a partir da interface com a camada de óxido é expresso por Xdmáx (2.1) [36].

$$
\mathrm{x}_{\mathrm{dmáx}}=\sqrt{\frac{4 \varepsilon_{\mathrm{Si}} \phi_{\mathrm{F}}}{\mathrm{qN}}}
$$

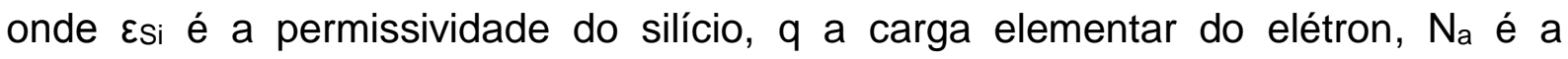
concentração de dopantes e фF é o potencial de Fermi (2.2) [36].

$$
\phi_{\mathrm{F}}=\frac{\mathrm{kT}}{\mathrm{q}} \ln \left(\frac{\mathrm{N}_{\mathrm{a}}}{\mathrm{n}_{\mathrm{i}}}\right)
$$

k é a constante de Boltzmann, T a temperatura em Kelvin e $\mathrm{n}_{\mathrm{i}}$ a concentração intrínseca. ni por sua vez também depende da temperatura (2.3) [36]:

$$
\mathrm{n}_{\mathrm{i}}=3,9 \times 10^{16} \mathrm{~T}^{\frac{3}{2}} \mathrm{e}^{-\left(\frac{\mathrm{E}_{\mathrm{g}}}{2 \mathrm{kT}}\right)}
$$


onde Eg é a largura da banda proibida.

Transistores parcialmente depletados (PD SOI) tem espessura do silício tsi maior que duas vezes o xdmáx. Essa condição garante que nunca haverá interação entre as regiões de depleção oriundas da primeira e da segunda interfaces .

Transistores totalmente depletados (FD SOI) tem espessura do silício tsi menor que o Xdmáx. Essa condição garante que o silício estará completamente depletado para valores de $\left|V_{G F}\right|$ acima de $\mathrm{V}_{\mathrm{T}}$, independentemente do valor de $\mathrm{V}_{\mathrm{GB}}$ (exceto para algumas condições de $V_{G B}$ na qual pode haver uma fina camada invertida ou acumulada na segunda interface). Os transistores FD SOI apresentam características mais atraentes que os PD SOI tais como campos elétricos menores e maiores transcondutância e melhor inclinação de sublimiar [36].

\subsubsection{Tensão de limiar $\left(V_{T}\right)$}

A tensão de limiar dos transistores SOI modo enriquecimento planares corresponde à tensão de porta $\bigvee_{G F}$ na qual a primeira interface atinge a inversão, ou seja, o potencial de superfície na primeira interface vale $\phi_{S 1}=2 \phi \mathrm{F}$.

\section{PD SOI:}

Nos transistores PD SOI não há interação entre a primeira e a segunda interface e, portanto, a tensão de limiar pode ser expressa da mesma maneira que para transistores MOSFET convencionais. A expressão para $\mathrm{V}_{\mathrm{T}}$ no caso de um substrato tipo p é dada por (2.4) [10].

$$
\mathrm{V}_{\mathrm{T}}=\mathrm{V}_{\mathrm{FB} 1}+2 \phi_{\mathrm{F}}+\frac{\mathrm{qN}_{\mathrm{a}} \mathrm{x}_{\mathrm{dmáx}}}{\mathrm{C}_{\mathrm{ox} 1}}
$$

onde $\mathrm{C}_{\mathrm{ox} 1}$ é a capacitância por unidade de área do óxido de porta e $\mathrm{V}_{\mathrm{FB} 1}$ é a tensão de banda plana dadas pelas expressões (2.5) e (2.6), respectivamente.

$$
\begin{gathered}
\mathrm{C}_{\mathrm{ox} 1}=\frac{\varepsilon_{\mathrm{ox}}}{\mathrm{t}_{\mathrm{ox}}} \\
\mathrm{V}_{\mathrm{FB} 1}=\Phi_{\mathrm{MS} 1}-\frac{\mathrm{Q}_{\mathrm{ox} 1}}{\mathrm{C}_{\mathrm{ox} 1}}
\end{gathered}
$$


onde $\varepsilon_{o x}$ é a permissividade do óxido, $\Phi_{M S 1}$ é a diferença de função trabalho entre o metal de porta e o semicondutor e Qox1 é a densidade efetiva de cargas no óxido de porta por unidade de área.

\section{FD SOI:}

Nos transistores FD SOI há interação entre a primeira e segunda interfaces. Logo a tensão $V_{G F}$ irá causar efeitos em ambas as interfaces, assim como a tensão $V_{\text {GB. }}$ O modelo Lim \& Fossum [37] descreve o acoplamento das cargas na primeira e segunda interface, conforme (2.7) e (2.8) [10].

$$
\begin{aligned}
& \mathrm{V}_{\mathrm{GF}}=\Phi_{\mathrm{MS} 1}-\frac{\mathrm{Q}_{\mathrm{ox} 1}}{\mathrm{C}_{\mathrm{ox} 1}}+\left(1+\frac{\mathrm{C}_{\mathrm{Si}}}{\mathrm{C}_{\mathrm{ox} 1}}\right) \phi_{\mathrm{S} 1}-\frac{\mathrm{C}_{\mathrm{Si}}}{\mathrm{C}_{\mathrm{ox} 1}} \phi_{\mathrm{S} 2}-\frac{\frac{1}{2 \mathrm{Q}_{\mathrm{depl}}}+\mathrm{Q}_{\mathrm{inv} 1}}{\mathrm{C}_{\mathrm{ox} 1}} \\
& \mathrm{~V}_{\mathrm{GB}}=\Phi_{\mathrm{MS} 2}-\frac{\mathrm{Q}_{\mathrm{ox} 2}}{\mathrm{C}_{\mathrm{BOX}}}+\left(1+\frac{\mathrm{C}_{\mathrm{Si}}}{\mathrm{C}_{\mathrm{BOX}}}\right) \phi_{\mathrm{S} 2}-\frac{\mathrm{C}_{\mathrm{Si}}}{\mathrm{C}_{\mathrm{BOX}}} \phi_{\mathrm{S} 1}-\frac{\frac{1}{2 \mathrm{Q}_{\mathrm{depl}}}+\mathrm{Q}_{\mathrm{S} 2}}{\mathrm{C}_{\mathrm{BOX}}}
\end{aligned}
$$

onde $C_{s i}$ é a capacitância por unidade de área do silício, $\phi s 1$ é o potencial de superfície na primeira interface, $\phi s 2$ é o potencial de superfície na segunda interface, Qdepl é a carga de depleção do silício por unidade de área, Qinv1 é a carga de inversão na primeira interface por unidade de área, $\Phi_{\mathrm{MS} 2}$ é a diferença de função trabalho entre o substrato e a camada de silício, Qox2 é a densidade efetiva de cargas no óxido enterrado por unidade de área, $\mathrm{C}_{\text {во }}$ é a capacitância por unidade de área do óxido enterrado dado por (2.9) e Qs2 é carga na segunda interface por unidade de área.

$$
\mathrm{C}_{\mathrm{BOX}}=\frac{\varepsilon_{\mathrm{ox}}}{\mathrm{t}_{\mathrm{BOX}}}
$$

A tensão de limiar irá depender da condição na segunda interface. Podemos distinguir os seguintes casos: 
- Segunda interface acumulada

Nesse caso podemos aproximar $\phi$ s2 para 0 e a carga de inversão também 0 . A tensão de limiar corresponde a condição em que $\phi S 1=2 \phi \mathrm{F}$. Substituindo esses valores nas equações (2.7) e (2.8), encontramos uma expressão para $\mathrm{V}_{\mathrm{T}, \mathrm{ac2}}(2.10)$.

$$
\mathrm{V}_{\mathrm{T}, \mathrm{ac} 2}=\Phi_{\mathrm{MS} 1}-\frac{\mathrm{Q}_{\mathrm{ox} 1}}{\mathrm{C}_{\mathrm{ox} 1}}+\left(1+\frac{\mathrm{C}_{\mathrm{Si}}}{\mathrm{C}_{\mathrm{ox} 1}}\right) 2 \phi_{\mathrm{F}}-\frac{\mathrm{Q}_{\mathrm{depl}}}{2 \mathrm{C}_{\mathrm{ox} 1}}
$$

- Segunda interface invertida

Nesse caso podemos aproximar $\phi \mathrm{S} 2$ para $2 \phi \mathrm{F}$, a carga de inversão para 0 e $\phi \mathrm{S} 1$

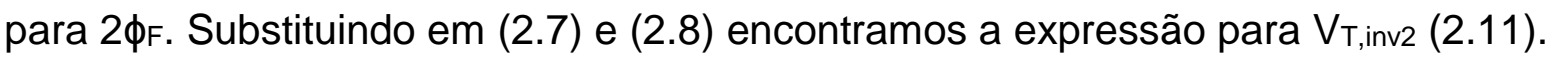

$$
\mathrm{V}_{\mathrm{T}, \mathrm{inv} 2}=\Phi_{\mathrm{MS} 1}-\frac{\mathrm{Q}_{\mathrm{ox} 1}}{\mathrm{C}_{\mathrm{ox} 1}}+2 \phi_{\mathrm{F}}-\frac{\mathrm{Q}_{\mathrm{depl}}}{2 \mathrm{C}_{\mathrm{ox} 1}}
$$

A segunda interface invertida faz com que haja corrente nessa condição de

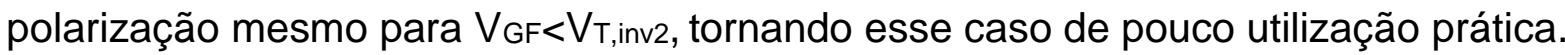

- Segunda interface depletada

Nesse caso, o valor do potencial de superfície na segunda interface está no intervalo $0<\phi \mathrm{S} 2<2 \phi \mathrm{F}$. Considerando $\phi_{\mathrm{S} 1}=2 \phi_{\mathrm{F}}$ e Qinv1 $=\mathrm{QS}_{\mathrm{s} 2}=0$ nas equações $(2.7)$ e (2.8) temos a expressão (2.12).

$$
\mathrm{V}_{\mathrm{T}, \mathrm{depl} 2}=\mathrm{V}_{\mathrm{T}, \mathrm{ac} 2}-\frac{\mathrm{C}_{\mathrm{Si}} \mathrm{C}_{\mathrm{BOX}}}{\mathrm{C}_{\mathrm{ox} 1}\left(\mathrm{C}_{\mathrm{Si}}+\mathrm{C}_{\mathrm{BOX}}\right)}\left(\mathrm{V}_{\mathrm{GB}}-\mathrm{V}_{\mathrm{GB}, \mathrm{ac} 2}\right)
$$

onde $V_{\mathrm{GB}, \text { ac2 }}$ é dado pela equação (2.13) para $\varphi_{\mathrm{S} 1}=2 \varphi_{\mathrm{F}}, \varphi_{\mathrm{S} 2}=0$ e $\mathrm{Qs}_{\mathrm{S} 2}=0$.

$$
\mathrm{V}_{\mathrm{GB}, \mathrm{ac} 2}=\Phi_{\mathrm{MS} 2}-\frac{\mathrm{Q}_{\mathrm{ox} 2}}{\mathrm{C}_{\mathrm{BOX}}}-\frac{\mathrm{C}_{\mathrm{Si}}}{\mathrm{C}_{\mathrm{BOX}}} 2 \phi_{\mathrm{F}}-\frac{\mathrm{Q}_{\mathrm{depl}}}{2 \mathrm{C}_{\mathrm{BOX}}}
$$

\subsubsection{Inclinação de sublimiar (S)}

Este parâmetro é definido como sendo o inverso da inclinação da curva IDXVGF no regime sublimiar na escala logarítimica. Quanto menor o valor de $\mathrm{S}$, mais rápido é a transição entre o estado ligado e desligado do transistor. 


$$
\mathrm{S}=\frac{\mathrm{dV}_{\mathrm{GF}}}{\mathrm{d}\left(\log \mathrm{I}_{\mathrm{D}}\right)} \quad[\mathrm{mV} / \mathrm{dec}]
$$

Desprezando-se as capacitâncias de armadilhas de interface, a inclinação de sublimiar pode ser aproximada por [10]:

$$
\mathrm{S}=\frac{\mathrm{kT}}{\mathrm{q}} \mathrm{n} \ln (10)
$$

onde $n=1+\alpha$ é o fator de corpo, sendo $\alpha$ a razão entre a capacitância vista entre o canal e o substrato dividida pela capacitância entre o canal e a porta no modelo capacitivo do canal mostrado na Figura 2.3. Para transistor MOSFET convencionais $\alpha=\frac{C_{D}}{C_{o x}}=\frac{\varepsilon_{S i}}{x_{d m a x x} C_{o x}}$ (onde $C_{D}$ é a capacitância de depleção), já para transistor SOI com a segunda interface acumulada $\alpha=\frac{\mathrm{C}_{\mathrm{Si}}}{\mathrm{C}_{\mathrm{ox} 1}}=\frac{\varepsilon_{\mathrm{Si}}}{\mathrm{t}_{\mathrm{Si}} \mathrm{C}_{\mathrm{ox} 1}}$ e para transistor SOI com a segunda interface depletada $\alpha=\frac{\mathrm{C}_{\mathrm{Si}} \mathrm{C}_{\mathrm{BOX}}}{\mathrm{C}_{\mathrm{Ox} 1}\left(\mathrm{C}_{\mathrm{Si}_{\mathrm{i}}}+\mathrm{C}_{\mathrm{BOX}}\right)}$.

Figura 2.3 - Modelo de capacitâncias entre porta e substrato. a) MOS convencional. b) FD SOI MOSFET.

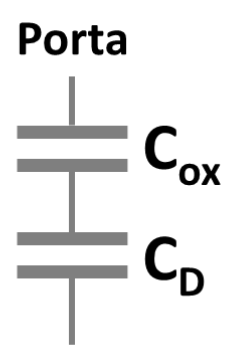

Substrato

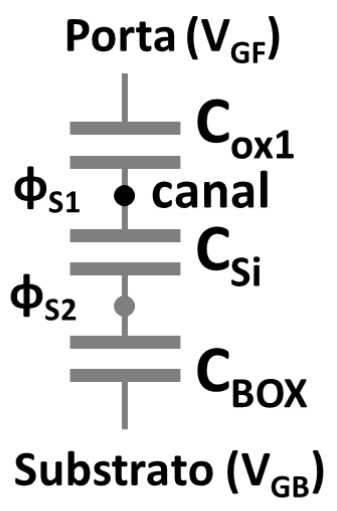

b) FD SOI MOSFET

\section{a) MOS convencional}

Fonte: Autor.

Tipicamente o valor de a para SOI com segunda interface depletada é menor que o valor de a para MOSFET convencional, que por sua vez é menor que o a para SOI MOSFET com segunda interface acumulada ( $\left.\alpha_{F D S O I}<\alpha_{\text {conv. }}<\alpha_{F D S O l a c c}\right)$. Logo os transistores SOI MOSFET com a segunda interface depletada possuem o menor valor para inclinação de sublimiar sendo, portanto, mais rápidos. Outra vantagem é permitir usar valores de tensão de limiar menores sem aumentar muito a corrente de fuga quando o transistor está desligado. 


\subsubsection{Efeito de corpo na tensão de limiar}

No caso dos transistores parcialmente depletados, a tensão de limiar não varia com $V_{G B}$ pois não existe acoplamento entre o eletrodo de porta e o substrato.

No caso dos transistores totalmente depletados, podemos encontrar a variação da tensão de limiar com $V_{G B}$ através das expressões (2.16), (2.17) e (2.18) nas condições de segunda interface acumulada, invertida e depletada respectivamente [10].

$$
\begin{gathered}
\frac{d V_{T, \text { ac } 2}}{d V_{G B}}=0 \\
\frac{d V_{T, \text { inv } 2}}{d V_{G B}}=0 \\
\frac{d V_{T, \text { depl2 }}}{d V_{G B}}=-\frac{C_{S i} C_{B O X}}{C_{\text {ox } 1}\left(C_{S i} C_{B O X}\right)}=\frac{-\varepsilon_{S i} C_{B O X}}{C_{\text {ox } 1}\left(t_{S i} C_{\text {ox } 2}+\varepsilon_{S i}\right)}=-\alpha
\end{gathered}
$$

\subsubsection{Transcondutância}

A transcondutância é um parâmetro que mede a efetividade do controle da corrente de dreno pela tensão de porta $\mathrm{V}_{\mathrm{GF}}$.

$$
\mathrm{gm}=\frac{\mathrm{dI}_{\mathrm{D}}}{\mathrm{dV}_{\mathrm{GF}}}
$$

A transcondutância pode ser expressa pelas equações (2.20) e (2.21) para as regiões de saturação e triodo, respectivamente.

$$
\begin{gathered}
\mathrm{gm}_{\mathrm{sat}}=\frac{\mu_{\mathrm{ef}} \mathrm{C}_{\mathrm{ox} 1} \mathrm{~W}}{\mathrm{~nL}}\left(\mathrm{~V}_{\mathrm{GF}}-\mathrm{V}_{\mathrm{T}}\right) \\
\mathrm{gm}_{\text {triodo }}=\frac{\mu_{\mathrm{ef}} \mathrm{C}_{\mathrm{ox} 1} \mathrm{~W}}{\mathrm{~L}} \mathrm{~V}_{\mathrm{DS}}
\end{gathered}
$$

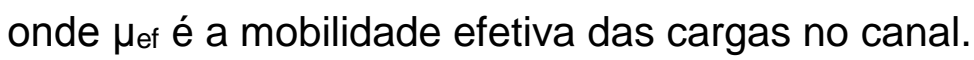




\subsection{Ponto invariante com a temperatura (ZTC)}

A temperatura influencia diversos parâmetros tais como o potencial de Fermi фF e a mobilidade. É desejável que a corrente dos transistores não sofra grandes variações em função da temperatura, para que não haja mudanças de polarização dos circuitos e para que eles continuem funcionando corretamente.

Figura 2.4 - Exemplo de curvas de transferência de um transistor MOS tipo p que apresenta o ponto de ZTC.

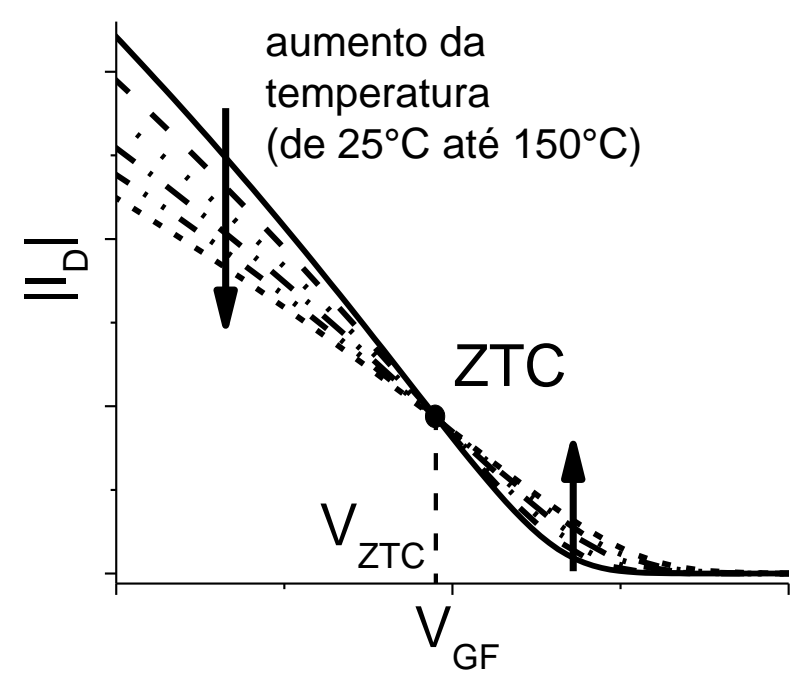

Fonte: Autor.

O ponto de polarização na Figura 2.4 em que a corrente de dreno não varia com a temperatura é conhecido como Ponto Invariante com a Temperatura (ZTC Zero Temperature Coefficient) definido pela expressão (2.22) [38]. Essa condição de polarização é muito importante, especialmente para aplicações de circuitos integrados analógicos, para manter a estabilidade do circuito e é tipicamente observado em transistores MOSFET [39], SOI MOSFET parcialmente depletados [40] e dispositivos similares [41]. Porém, sabe-se que a presença ou a estabilidade do ZTC não é observado em todos os tipos de transistores [42] [43].

$$
\left.\frac{\mathrm{dI}}{\mathrm{dT}}\right|_{\mathrm{V}_{\mathrm{GF}=\mathrm{V}_{\mathrm{ZTC}}}}=0
$$

O ponto de polarização ZTC surge a partir da compensação de dois fenômenos causados pelo aumento da temperatura. O primeiro é a dependência do potencial de Fermi com a temperatura, que por sua vez influenciará no valor da tensão de limiar. O aumento de temperatura causa a diminuição (em módulo) da tensão de limiar, e 
consequentemente, o aumento da corrente de dreno. O segundo fenômeno é a degradação da mobilidade, que por sua vez causará a diminuição da corrente de dreno [38].

\subsubsection{Modelo para $V_{\mathrm{ZTC}}$ na região linear}

Foi utilizado o modelo Camillo-Martino para determinar VzTc na região linear de operação do transistor de forma simples, analisando os principais parâmetros que influenciam no seu valor [38] [44]. O modelo será demonstrado a seguir.

A equação para a corrente de dreno de um transistor SOI MOSFET é dada por (2.23).

$$
\mathrm{I}_{\mathrm{D}}=\frac{\mu \mathrm{C}_{\mathrm{ox} 1} \mathrm{~W}}{\mathrm{~L}}\left[\left(\mathrm{~V}_{\mathrm{GF}}-\mathrm{V}_{\mathrm{T}}\right) \mathrm{V}_{\mathrm{DS}}-\frac{\mathrm{nV} \mathrm{V}_{\mathrm{DS}}^{2}}{2}\right]
$$

onde $\mu$ é a mobilidade efetiva definida a partir da mobilidade de baixo campo $\mu_{0}, 0$ fator de degradação da mobilidade $\theta$, a tensão de porta $V_{G F}$ e a tensão de limiar $V_{T}$.

$$
\mu=\frac{\mu_{0}}{1+\frac{\theta}{n}\left(V_{G F}-V_{T}\right)}
$$

É possível determinar duas expressões, equações (2.25) e (2.26), para a corrente de dreno para duas temperaturas distintas $T_{1}$ e $T_{2}>T_{1}$ a partir das equações (2.23) e (2.24). O ponto de ZTC é por definição a condição em que a corrente se mantém constante, portanto igualando as duas expressões da corrente para as duas temperaturas é possível encontrar o valor da tensão de porta correspondente à VzTc.

$$
\begin{aligned}
& \mathrm{I}_{\mathrm{D}(\mathrm{T} 1)}=\frac{\mu_{0(\mathrm{~T} 1)}}{1+\frac{\theta_{1}}{\mathrm{n}_{1}}\left(\mathrm{~V}_{\mathrm{GF}}-\mathrm{V}_{\mathrm{T} 1}\right)} \frac{\mathrm{C}_{\mathrm{ox} 1} \mathrm{~W}}{\mathrm{~L}}\left[\left(\mathrm{~V}_{\mathrm{GF}}-\mathrm{V}_{\mathrm{T} 1}\right) \mathrm{V}_{\mathrm{DS}}-\frac{\mathrm{n}_{1} \mathrm{~V}_{\mathrm{DS}}^{2}}{2}\right] \\
& \mathrm{I}_{\mathrm{D}(\mathrm{T} 2)}=\frac{\mu_{0(\mathrm{~T} 2)}}{1+\frac{\theta_{2}}{\mathrm{n}_{2}}\left(\mathrm{~V}_{\mathrm{GF}}-\mathrm{V}_{\mathrm{T} 2}\right)} \frac{\mathrm{C}_{\mathrm{Ox} 1} \mathrm{~W}}{\mathrm{~L}}\left[\left(\mathrm{~V}_{\mathrm{GF}}-\mathrm{V}_{\mathrm{T} 2}\right) \mathrm{V}_{\mathrm{DS}}-\frac{\mathrm{n}_{2} \mathrm{~V}_{\mathrm{DS}}^{2}}{2}\right]
\end{aligned}
$$

onde $\mu_{0(\mathrm{~T} 1)}$ e $\mu_{0 \text { (T2) }}$ são as mobilidades de baixo campo, $\theta_{1}$ e $\theta_{2}$ são os coeficientes de degradação da mobilidade, $\mathrm{n}_{1}$ e $\mathrm{n}_{2}$ são os fatores de corpo, $\mathrm{V}_{\mathrm{T} 1}$ e $\mathrm{V}_{\mathrm{T} 2}$ são as tensões 
de limiar e $I_{D(T 1)}$ e $I_{D(T 2)}$ são as correntes de dreno para as temperaturas $T_{1}$ e $T_{2}$ respectivamente.

Adotando as seguintes simplificações:

$$
\begin{aligned}
& \mathrm{n}_{1}=\mathrm{n}_{2}=\mathrm{n} \\
& \theta_{1}=\theta_{2}=\theta
\end{aligned}
$$

é possível chegar na expressão para VZTC, dado pela equação (2.29).

$$
\mathrm{V}_{\mathrm{ZTC}}=\frac{\mu_{1} \mathrm{~V}_{\mathrm{T} 1}-\mu_{2} \mathrm{~V}_{\mathrm{T} 2}}{\mu_{1}-\mu_{2}}+\frac{\mathrm{nV}_{\mathrm{DS}}}{2}
$$

onde $\mu_{1}$ e $\mu_{2}$ são as mobilidades efetivas nas temperatura T1 e T2, respectivamente.

Utilizando a relação da mobilidade com a temperaturas (2.30) e a variação da tensão de limiar com a temperatura (2.31) chegamos na expressão final do VZTC na região de triodo (2.32) [38].

$$
\begin{gathered}
\frac{\mu_{1}}{\mu_{2}}=\left(\frac{\mathrm{T}_{2}}{\mathrm{~T}_{1}}\right)^{\mathrm{c}} \\
\frac{\mathrm{V}_{\mathrm{T} 2}-\mathrm{V}_{\mathrm{T} 1}}{\mathrm{~T}_{2}-\mathrm{T}_{1}}=\frac{\Delta \mathrm{V}_{\mathrm{T}}}{\Delta \mathrm{T}}
\end{gathered}
$$

onde c é o coeficiente de degradação da mobilidade com a temperatura.

$$
\mathrm{V}_{\mathrm{ZTC}}=\frac{\mathrm{V}_{\mathrm{T} 1}-\left(\frac{\mathrm{T}_{1}}{\mathrm{~T}_{2}}\right)^{\mathrm{c}}\left(\mathrm{V}_{\mathrm{T} 1}+\frac{\Delta \mathrm{V}_{\mathrm{TH}}}{\Delta \mathrm{T}} \cdot\left(\mathrm{T}_{2}-\mathrm{T}_{1}\right)\right)}{1-\left(\frac{\mathrm{T}_{1}}{\mathrm{~T}_{2}}\right)^{\mathrm{c}}}+\frac{\mathrm{nV} \mathrm{DS}}{2}
$$

\subsection{Contato metal-semicondutor}

Há dois tipos de contatos entre metal e semicondutor, os contatos Ôhmico e os contatos Schottky. 


\subsubsection{Contato Ôhmico}

O contato Ôhmico é um contato não retificador e que segue a lei de Ohm.

No caso em que o nível de Fermi do metal (EFM) for maior que o nível de Fermi do semicondutor $\left(\mathrm{E}_{\mathrm{F}}\right)$, as bandas de energia do semicondutor tipo $\mathrm{n}$ se curvarão para baixo, conforme mostrado na Figura 2.5. Esse encurvamento é pequeno e como resultado não há uma barreira de potencial entre o metal e o semicondutor. Os elétrons fluem livremente através do contato [36].

Figura 2.5 - Diagrama de bandas de energia para $E_{F M}>E_{F}$, formando uma junção Ôhmica entre metal e semicondutor, antes e depois do contato.

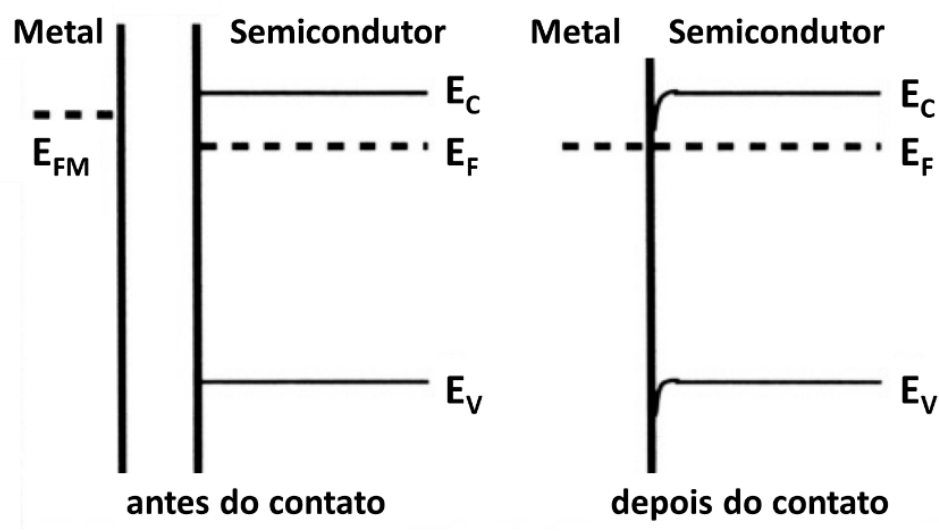

Fonte: adaptado de [36].

No caso em que $E_{F M}<E_{F}$, a princípio o contato formado seria do tipo Schottky, como será descrito a seguir. Porém se o semicondutor for altamente dopado (concentração de dopantes maior que $10^{20} \mathrm{~cm}^{-3}$ ), na prática o contato se comportará como sendo Ôhmico. A profundidade de depleção xd se torna pequena o suficiente para os elétrons tunelarem através da barreira de potencial (Figura 2.6).

Figura 2.6 - Diagrama de bandas de energia para $\mathrm{E}_{\mathrm{FM}}<\mathrm{E}_{\mathrm{F}}$ e semicondutor fortemente dopado, se comportando como contato Ôhmico.

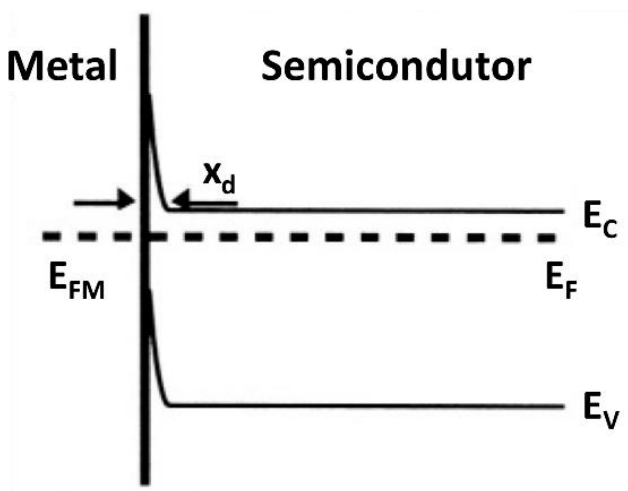

Fonte: adaptado de [36]. 


\subsubsection{Contato Schottky}

O contato Schottky é um contato retificador.

Considerando um semicondutor tipo n e que $E_{F M}<E_{F}$, no contato entre o metal e o semicondutor há uma transferência de elétrons no equilíbrio. Como resultado temos uma região de depleção no semicondutor conforme mostrado na Figura 2.7. O encurvamento das bandas de energia do semicondutor resulta em uma barreira de potencial $V_{i}$ vista pelos elétrons do semicondutor. No lado do metal, os elétrons enxergam uma barreira de potencial $\phi b$, dado pela equação (2.33) [36].

$$
\phi_{\mathrm{b}}=\Phi \mathrm{m}-\chi=V_{\mathrm{i}}+\left(\mathrm{E}_{\mathrm{C}}-\mathrm{E}_{\mathrm{F}}\right) / \mathrm{q}
$$

sendo $\Phi_{\mathrm{m}}$ a função trabalho do metal e $\mathrm{X}$ a afinidade eletrônica do semicondutor.

Figura 2.7 - Diagrama de bandas de energia para $E_{F M}<E_{F}$, formando um contato Schottky entre metal e semicondutor.

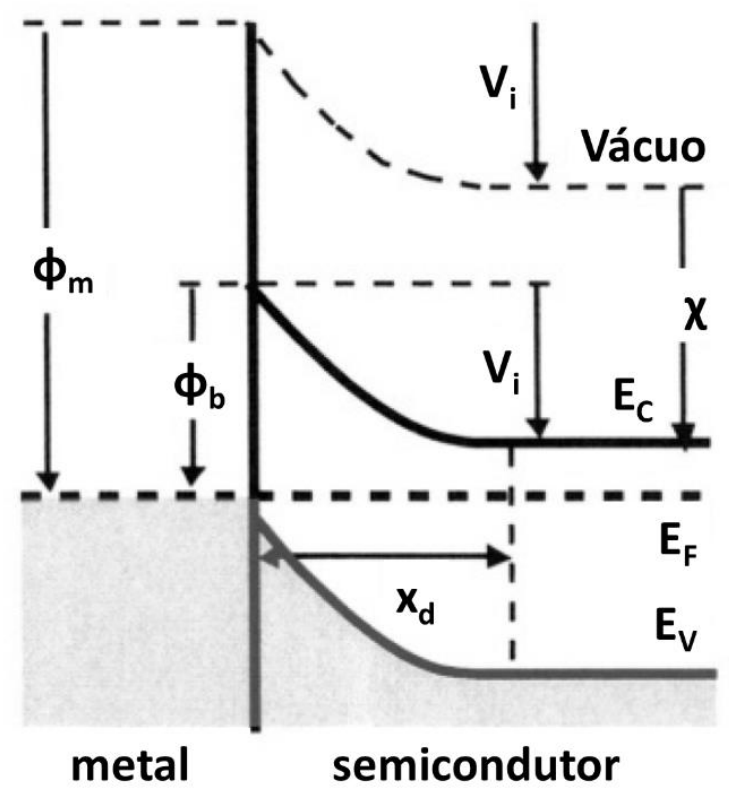

Fonte: adaptado de [36].

Se o contato for polarizado diretamente com uma tensão $V_{a}>0$, a barreira de potencial no lado do semicondutor diminui para $\mathrm{V}_{i}-\mathrm{V}_{a}$ (Figura 2.8A). Com isso uma grande quantidade de elétrons consegue atravessar essa barreira e, portanto, há corrente.

Caso contrário, se o contato for polarizado reversamente com uma tensão $\mathrm{V}_{\mathrm{a}}<0$, a barreira de potencial no lado do semicondutor aumenta para $\mathrm{V}_{\mathrm{i}} \mathrm{V}_{\mathrm{a}}$ (Figura 
2.8B). O fluxo de elétrons do semicondutor para o metal é bastante reduzido e é observado apenas uma pequena corrente de elétrons reversa do metal para o semicondutor.

Figura 2.8 - Diagrama de bandas de energia para um contato Schottky diretamente polarizado $(A)$ e reversamente polarizado $(\mathrm{B})$.

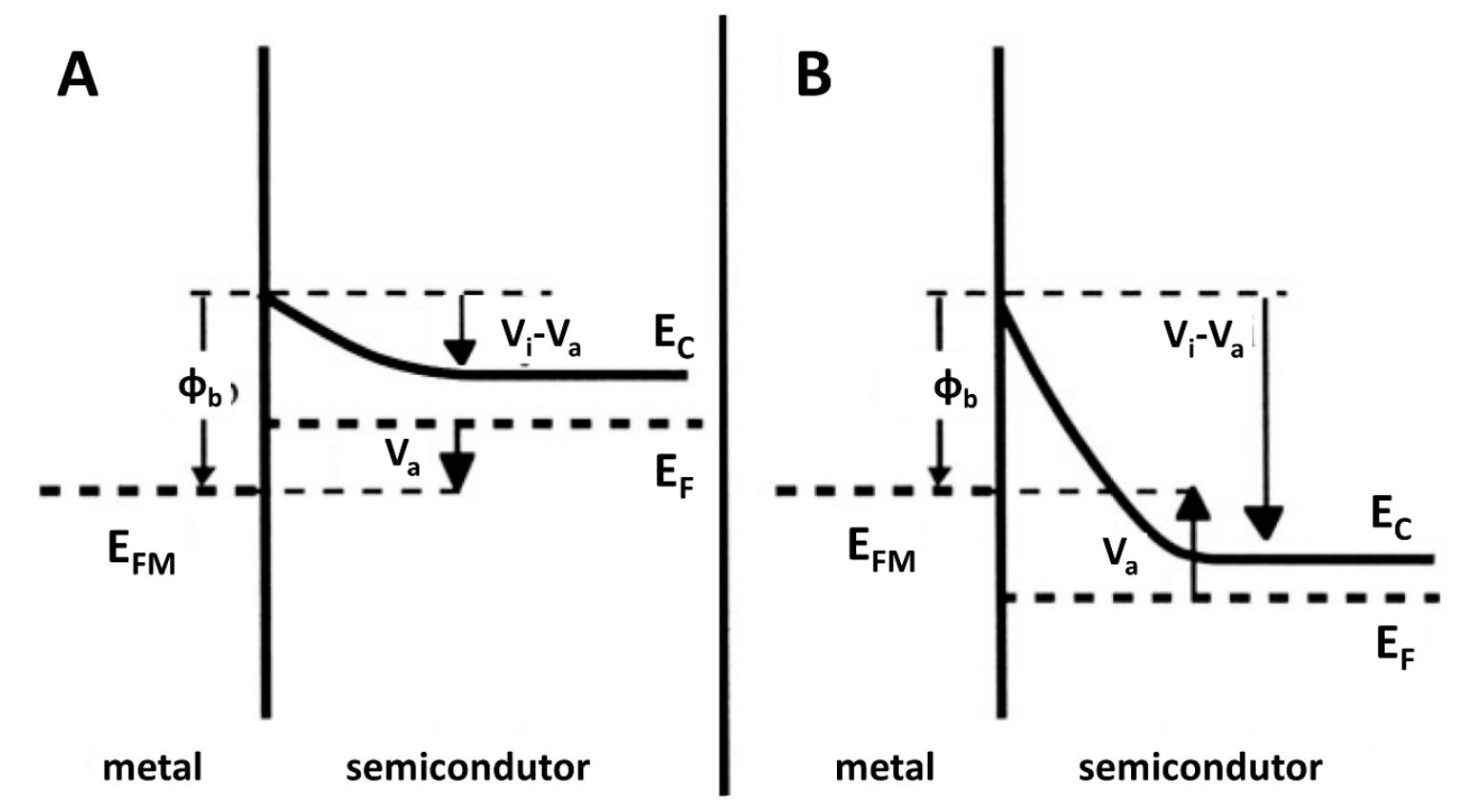

Fonte: adaptado de [36].

\subsection{Inversor CMOS}

O circuito inversor é muito importante para aplicações digitais. A partir do entendimento de suas propriedades é possível projetar estruturas mais complexas como outras portas lógicas, somadores, multiplicadores, etc. A tensão obtida na saída deste circuito possui o nível lógico oposto da tensão em sua entrada [45].

\subsubsection{Comportamento estático do inversor}

A Figura 2.9 mostra o esquema elétrico de um inversor CMOS utilizando um transistor tipo $n$ e um transistor tipo $p$, na qual $V_{D D}$ e GND representam as tensões de alimentação do circuito e $V_{\text {in }}$ e $V_{\text {out }}$ representam a tensão de entrada e de saída, respectivamente. Podemos compreender o seu funcionamento pensando nos transistores como chaves. Quando $\left|\mathrm{V}_{\mathrm{GF}}\right|<\left|\mathrm{V}_{\mathrm{T}}\right|$ os transistores estarão cortados e, 
portanto, possuirão uma resistência infinita (muito elevada). Quando $\left|V_{G F}\right|>\left|V_{T}\right|$ oS transistores estarão conduzindo e podemos associá-los uma pequena resistência.

Figura 2.9 - Circuito elétrico de um inversor CMOS.

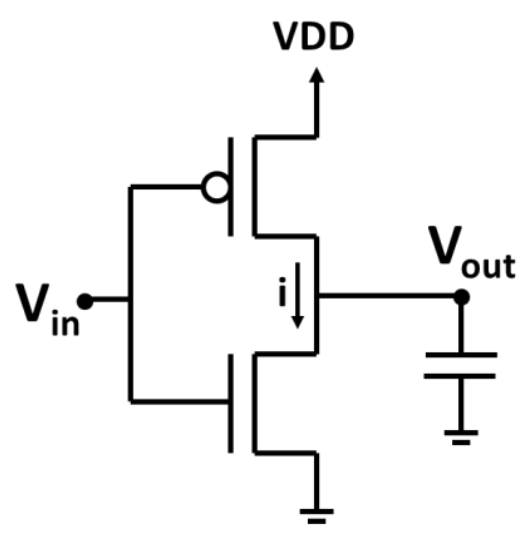

GND

Fonte: Autor.

Caso $V_{\text {in }}=V_{D D}$, o transistor tipo $n$ estará conduzindo enquanto o transistor tipo $p$ estará cortado. O circuito equivalente nesta situação pode ser visto na Figura 2.10a. Existe um caminho entre o nó de saída e o GND, resultando em $\mathrm{V}_{\text {out }}=0$.

Já no caso em que $\mathrm{V}_{\mathrm{in}}=0$, o oposto ocorre. $\mathrm{O}$ transistor tipo $\mathrm{n}$ passa a estar cortado enquanto o transistor tipo $\mathrm{p}$ está conduzindo. Pelo circuito equivalente mostrado na Figura 2.10b podemos ver que o resultado na saída é um $\mathrm{V}_{\text {out }}=\mathrm{V}_{\mathrm{DD}}$.

Figura 2.10 - Modelo de chaves do inversor CMOS
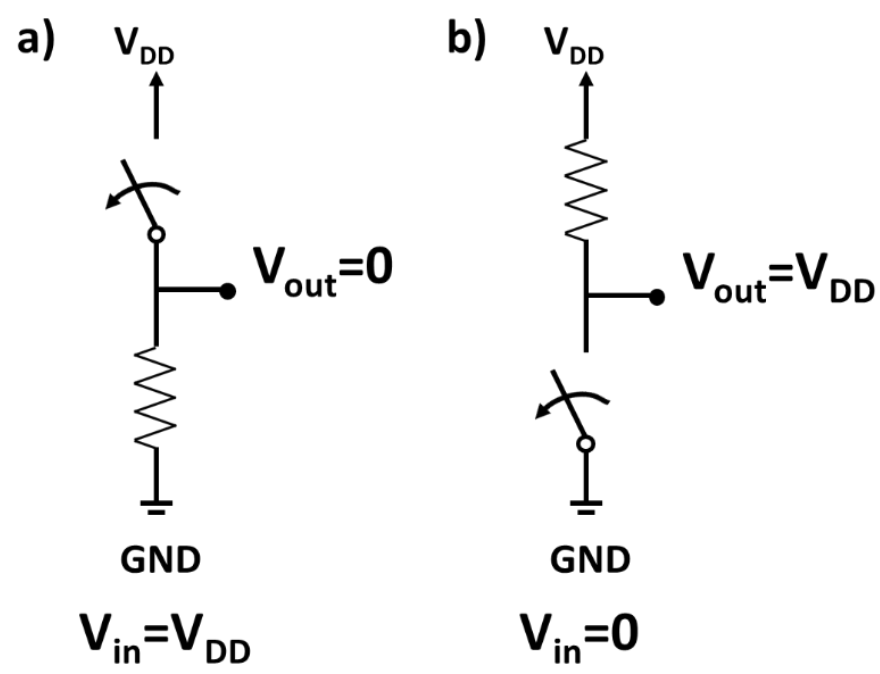

Fonte: Autor. 
Há sempre um caminho com resistência finita entre a saída do inversor com VDD ou GND, o que proporciona uma baixa impedância de saída para o circuito. Já a impedância de entrada é bastante elevada pois a entrada do inversor está conectada às portas dos transistores, que permanecem isoladas pelo óxido de porta.

Uma vantagem deste inversor é a ausência de corrente elétrica (desconsiderando momentos de transição e possíveis correntes de fuga), o que faz com que não haja consumo de potência.

A curva de transferência característica de um inversor CMOS pode ser vista na Figura 2.12. Podemos entender seu formato ao analisar as curvas de cargas do inversor apresentadas na

Figura 2.11. As curvas de corrente dos transistores tipo n e tipo $p$ são postas sobre as mesmas coordenadas, considerando que no circuito do inversor [45]:

$$
\begin{gathered}
\mathrm{I}_{\mathrm{Dn}}=-\mathrm{I}_{\mathrm{Dp}} \\
\mathrm{V}_{\mathrm{GFn}}=\mathrm{V}_{\text {in }} ; \mathrm{V}_{\mathrm{GFp}}=\mathrm{V}_{\text {in }}-\mathrm{V}_{\mathrm{DD}} \\
\mathrm{V}_{\mathrm{DSn}}=\mathrm{V}_{\text {out }} ; \mathrm{V}_{\mathrm{DSp}}=\mathrm{V}_{\text {out }}-\mathrm{V}_{\mathrm{DD}}
\end{gathered}
$$

Figura 2.11 - Curvas de carga para transistores nMOSFET e pMOSFET do circuito inversor CMOS.

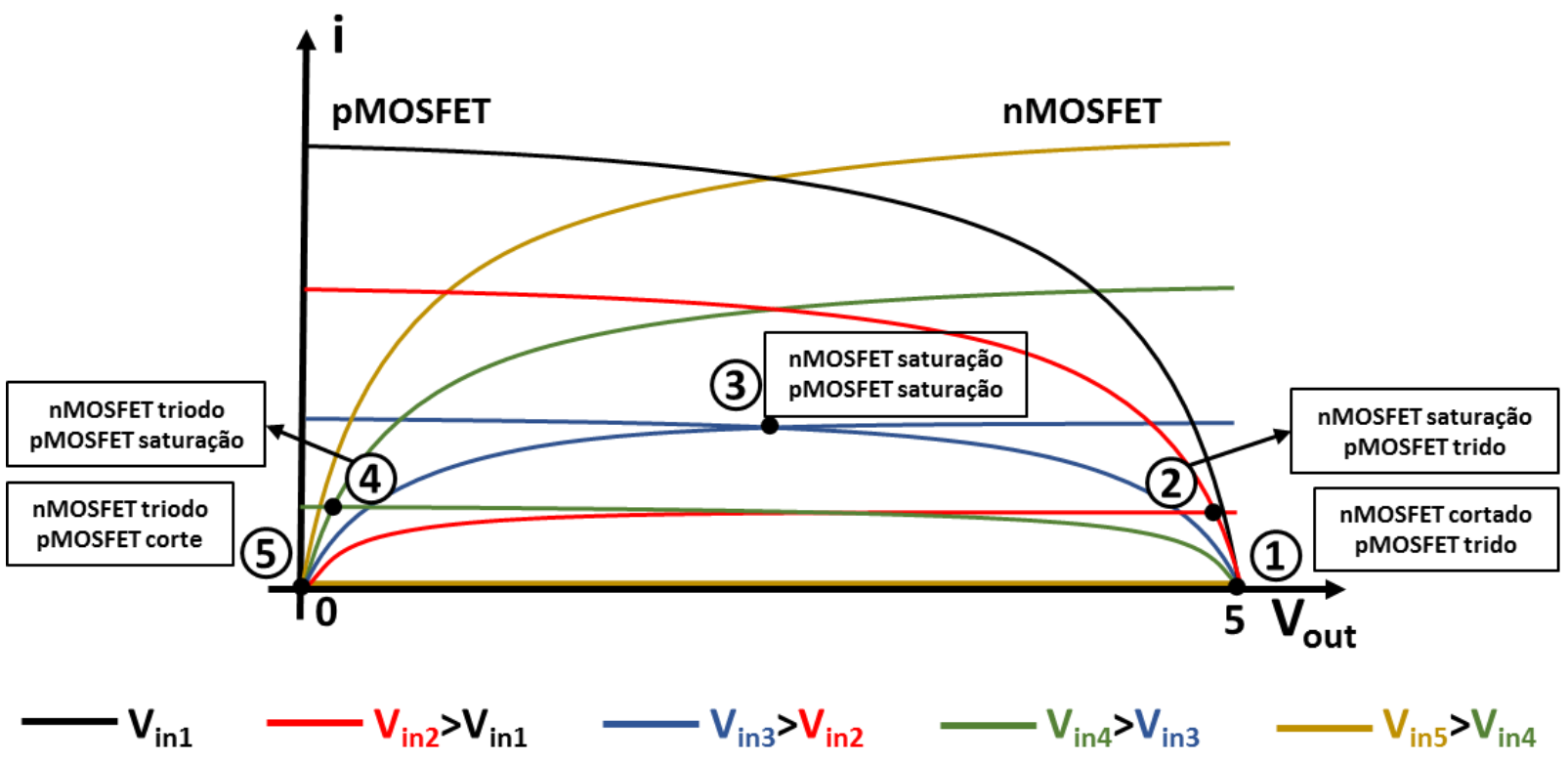

Fonte: Autor. 
$\mathrm{Na}$ operação estática do inversor, as correntes de dreno dos transistores nMOSFET e pMOSFET são iguais, o que graficamente corresponde aos pontos de intersecção destacados na

Figura 2.11 para uma mesma tensão de entrada.

Os pontos de 1 a 5 das curvas de cargas da

Figura 2.11 são condições de polarizações dentro das respectivas regiões $A$, $B, C, D$ e E na curva de transferência estática do inversor (Figura 2.12). Essa mesma figura mostra as condições de operação dos transistores nMOSFET e pMOSFET em cada regime da curva [45].

A região $A$ corresponde à $0<V_{i n}<V_{T n}$, sendo $V_{T n}$ a tensão de limiar do transistor tipo $n$. Portanto o transistor tipo $n$ está cortado $\left(V_{G S n}<V_{T n}\right)$, enquanto que o transistor tipo p está conduzindo na região de triodo (a tensão entre porta e fonte $-\mathrm{V}_{\mathrm{DD}}<\mathrm{V}_{\mathrm{GSp}}<\mathrm{V}_{T n}$ $\left.V_{D D}\right)$. Logo o potencial $V_{D D}$ será transmitido para a saída $\left(V_{\text {out }}=V_{D D}\right)$ e não há passagem de corrente pelo inversor.

Na região $\mathrm{B}$ o transistor tipo $\mathrm{P}$ continua na região de tríodo, mas o transistor tipo $\mathrm{n}$ entra na região de saturação. Nessa condição o transistor tipo $\mathrm{n}$ também passa a conduzir e há passagem de corrente no inversor. $\mathrm{O}$ aumento de $\mathrm{V}_{\text {in }}$ causa um aumento da corrente do transistor tipo n, provocando a redução da tensão de saída Vout.

A região $C$ corresponde a condição em que ambos transistores estão em saturação e a corrente no inversor é máxima. Uma pequena mudança na tensão de entrada resulta em uma grande variação da tensão de saída.

Na região $\mathrm{D}$, na qual $\mathrm{V}_{\mathrm{INV}}<\mathrm{V}_{\mathrm{in}}<\mathrm{V}_{\mathrm{DD}}-\left|\mathrm{V}_{\mathrm{Tp}}\right|$, o transistor tipo $\mathrm{n}$ passa a operar na região de triodo, enquanto o transistor tipo p opera na saturação.

Para $\mathrm{V}_{D D}-\left|\mathrm{V}_{T p}\right|<\mathrm{V}_{\text {in }}<\mathrm{V}_{\mathrm{DD}}$, correspondente a região $\mathrm{E}, 0<\mathrm{V}_{\mathrm{GSp}}<\left|\mathrm{V}_{T p}\right|$ e o transistor tipo p passa para o corte. Em compensação o transistor tipo $n$ estará conduzindo na região de triodo e a tensão na saída passa para $\mathrm{V}_{\text {out }}=0$, pois não há passagem de corrente no inversor. 
Figura 2.12 - Característica de transferência de tensão do inversor CMOS com transistores casados.

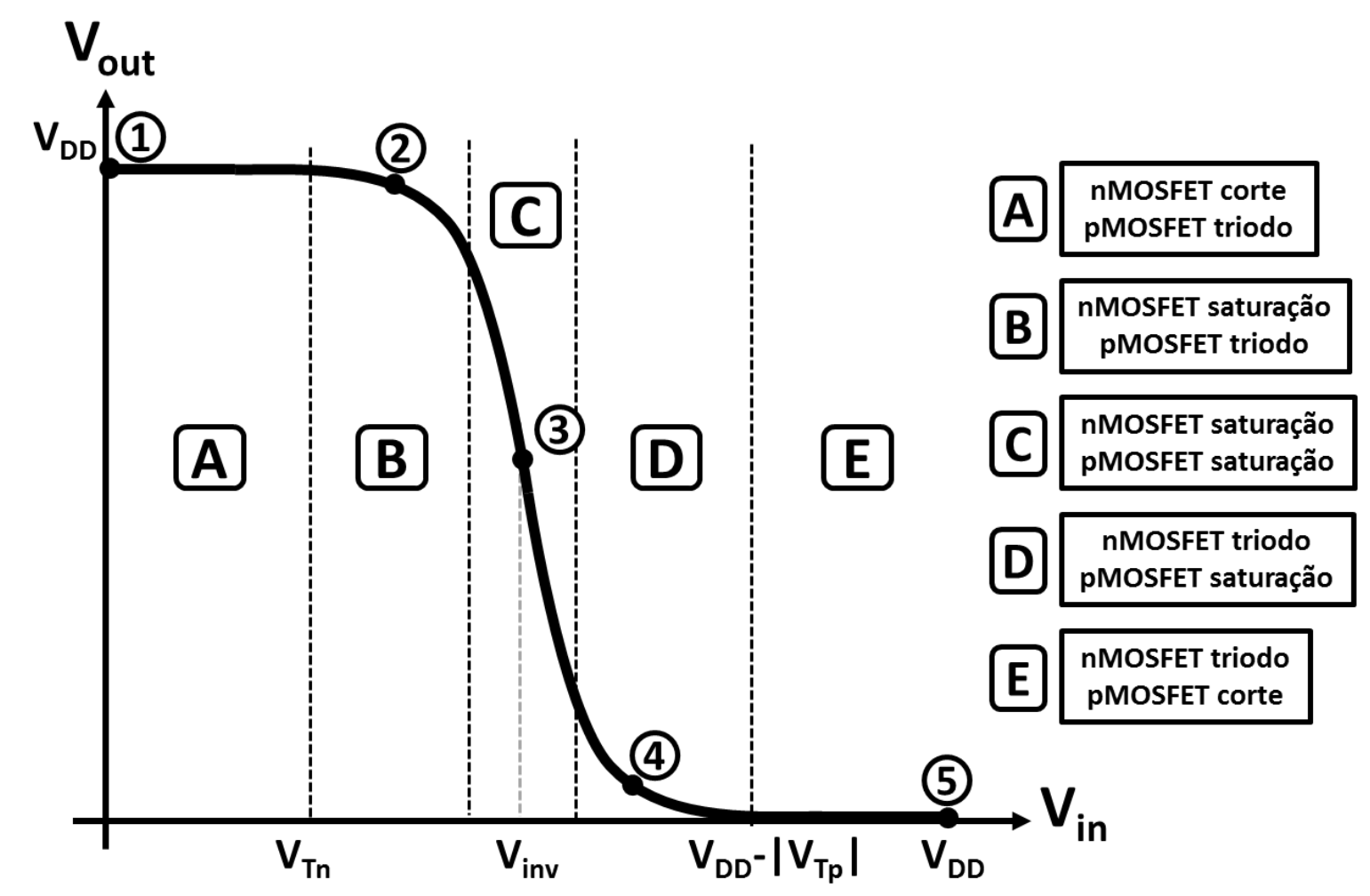

Fonte: Autor. 


\section{Materiais e métodos}

Neste capítulo são apresentados o conceito geral do dispositivo estudado, os equipamentos utilizados para realização das medidas elétricas, o simulador numérico e os métodos de extração dos parâmetros dos transistores analisados.

\subsection{Dispositivo estudado: BE SOI MOSFET}

O BE (Back Enhanced) é um transistor do tipo MOSFET construído em lâmina do tipo SOI (Silicon-On-Insulator) com porta de metal e que se destaca principalmente por dois motivos: (i) a ausência de processo de dopagem durante sua fabricação (consequentemente ausência de junções no silício). Ao contrário de transistores sem junção, porém com canal dopado [46], o BE SOI MOSFET não necessita de processo de dopagem; (ii) o fato de ser um transistor planar. Essas características tornam o processo de fabricação do BE SOI MOSFET bastante simples e mais acessível, bastando apenas três etapas de fotolitografia convencional para a definição dos transistores. Dispositivos com princípio de funcionamento similares propostos foram obtidos a partir de técnicas de fabricação mais sofisticadas e caras [23] [25] [26] [27] [33].

\subsubsection{Processo de fabricação}

Será apresentado nesta seção o processo de fabricação, de forma resumido, do BE SOI MOSFET. Os detalhes das etapas de fabricação não foram abordados neste trabalho.

A fabricação do BE SOI MOSFET inicia-se a partir de uma lâmina SOI com dopagem natural tipo $P$ com concentração de aproximadamente $10^{15} \mathrm{~cm}^{-3}$, orientação cristalográfica $<100>$ e espessura do óxido enterrado de $200 \mathrm{~nm}$. A sequência resumida dos processos de fabricação é descrita a seguir [31]:

1ํ) Oxidação térmica;

$2^{\circ}$ ) Primeira fotolitografia para definição da região ativa e da largura do canal (W), resultando no perfil apresentado na Figura 3.1a);

3ํ) Corrosão do $\mathrm{SiO}_{2}$ sobre a camada de silício; 
Figura 3.1 - a) Perfil do BE SOI MOSFET após a primeira fotolitografia. b) Perfil do BE SOI MOSFET após a segunda fotolitografia. c) Perfil do BE SOI MOSFET após a terceira fotolitografia.

a)

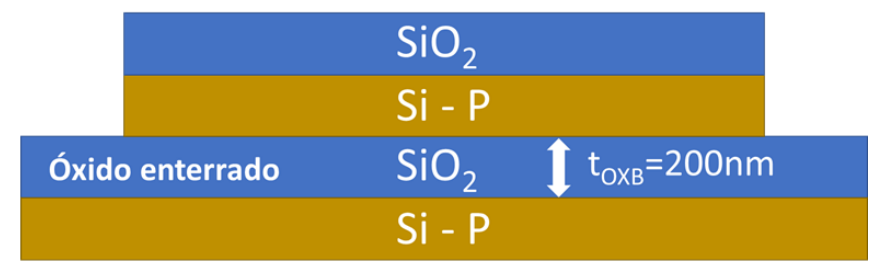

b)

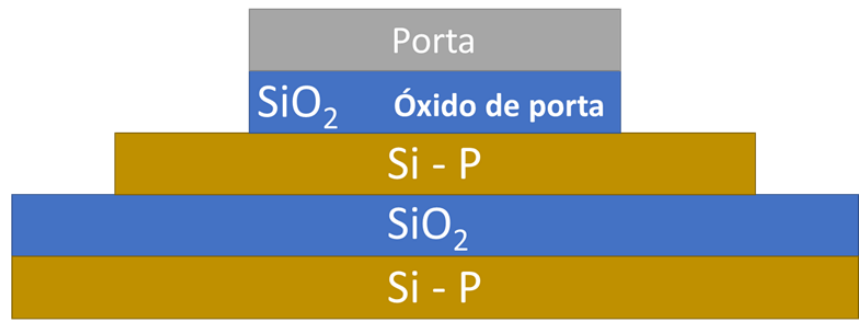

c)

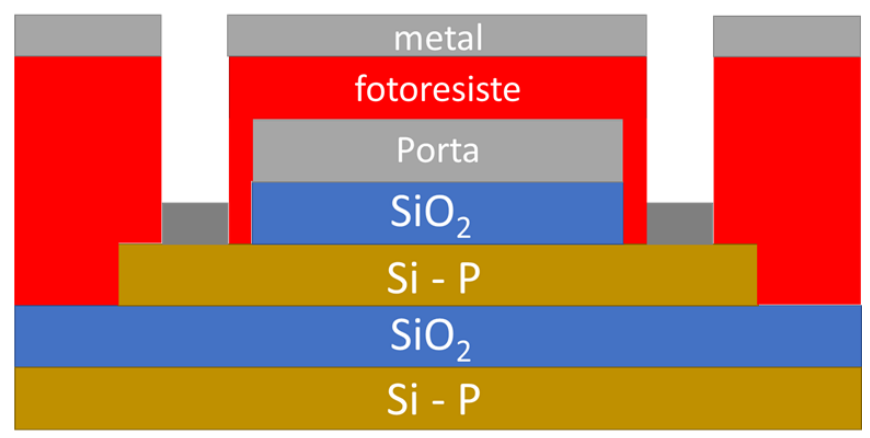

Fonte: adaptado de [31].

Figura 3.2 - Perfil esquemático do BE SOI MOSFET.

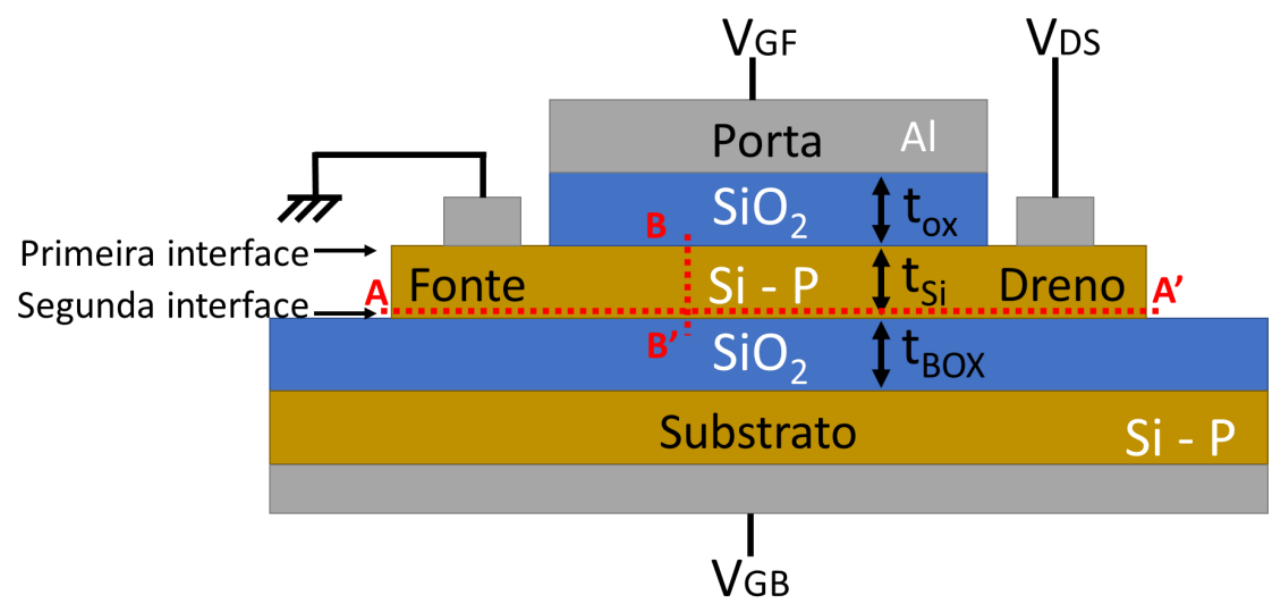

Fonte: Autor. 
4ํ) Óxido de porta;

5) Deposição da porta de alumínio;

$\left.6^{\circ}\right)$ Segunda fotolitografia para definição da porta e do comprimento do canal (L), resultando no perfil apresentado na Figura 3.1b);

$7^{\circ}$ ) Deposição do fotorresiste e realização da terceira fotolitografia para definição dos contatos;

$\left.8^{\circ}\right)$ Deposição do metal de contato para fonte e dreno, resultando no perfil apresentado na Figura 3.1c);

9ํ) Corrosão do fotorresiste e lift-off do metal sobre ele;

10)) Sinterização.

O perfil final do BE SOI MOSFET pode ser visto na Figura 3.2 no qual $V_{G F}$ representa a tensão no eletrodo de porta, $V_{G B}$ representa a tensão no substrato e $V_{D S}$ representa a tensão entre os eletrodos de dreno e fonte.

\subsubsection{Princípio de operação}

O BE SOI MOSFET apresenta um princípio de operação diferente dos transistores MOSFET convencionais. Seu funcionamento como transistor depende, entre outros fatores, principalmente das espessuras das camadas de óxido de porta (tox), silício (tsi) e óxido enterrado (tBox).

Ao aplicar uma tensão suficientemente elevada no substrato, o campo elétrico vertical gerado irá atrair cargas elétricas positivas (caso $V_{\mathrm{GB}}<0$ ) ou cargas elétricas negativas (caso $V_{G B}>0$ ) na interface camada de silício - óxido enterrado (segunda interface). Essa camada de cargas cria um caminho para que passe corrente elétrica entre fonte e dreno caso $V_{D S}$ seja diferente de 0 e $V_{G F}<V_{T p}$ (no caso de um transistor tipo p) ou $V_{G F}>V_{T n}$ (no caso de um transistor tipo n). Nesse caso o transistor está no estado ligado e diferente de transistores SOI convencionais, a corrente passa pela segunda interface ao invés da primeira. A Figura 3.3(a) representa o diagrama de bandas de energia e o perfil esquemático do dispositivo nesta condição de operação para o caso em que $V_{G B}<<0$ e, portanto, a camada de cargas na segunda interface é formada por lacunas. Já a Figura 3.3(b) representa as mesmas características para o caso em que $V_{G B}>>0$, ou seja, a camada de cargas na segunda interface agora é formada por elétrons. 
Caso a tensão de porta $\mathrm{V}_{\mathrm{GF}}$ seja maior que a tensão de limiar $\mathrm{V}_{\mathrm{Tp}}$ (no caso do transistor tipo p) ou caso $\mathrm{V}_{\mathrm{GF}}$ seja menor que a tensão de limiar $\mathrm{V}_{\mathrm{Tn}}$ (no caso do transistor tipo $n$ ), o campo elétrico originado pela porta é suficientemente elevado para interromper a camada de cargas na segunda interface diretamente abaixo do eletrodo de porta. Portanto a camada de silício fica totalmente depletada e a corrente entre fonte e dreno é interrompida. Nesse caso o transistor está no estado desligado. A Figura 3.3(c) representa o diagrama de bandas de energia nesta condição de operação, novamente para o caso em que $\mathrm{V}_{\mathrm{GB}}<<0$ (pMOS) e a Figura 3.3(d) representa o caso em que $V_{G B}>>0$ (nMOS). 
Figura 3.3 - Diagrama de bandas de energia esquemático mostrando o princípio de operação. a) BE SOI pMOSFET no estado ligado e a formação da camada de lacunas na segunda interface. b) BE SOI nMOSFET no estado ligado e a formação da camada de elétrons na segunda interface. c) BE

SOI pMOSFET no estado cortado e a região depletada interrompendo a camada de lacunas na segunda interface. d) do BE SOI nMOSFET no estado cortado e a região depletada interrompendo a camada de elétrons na segunda interface.
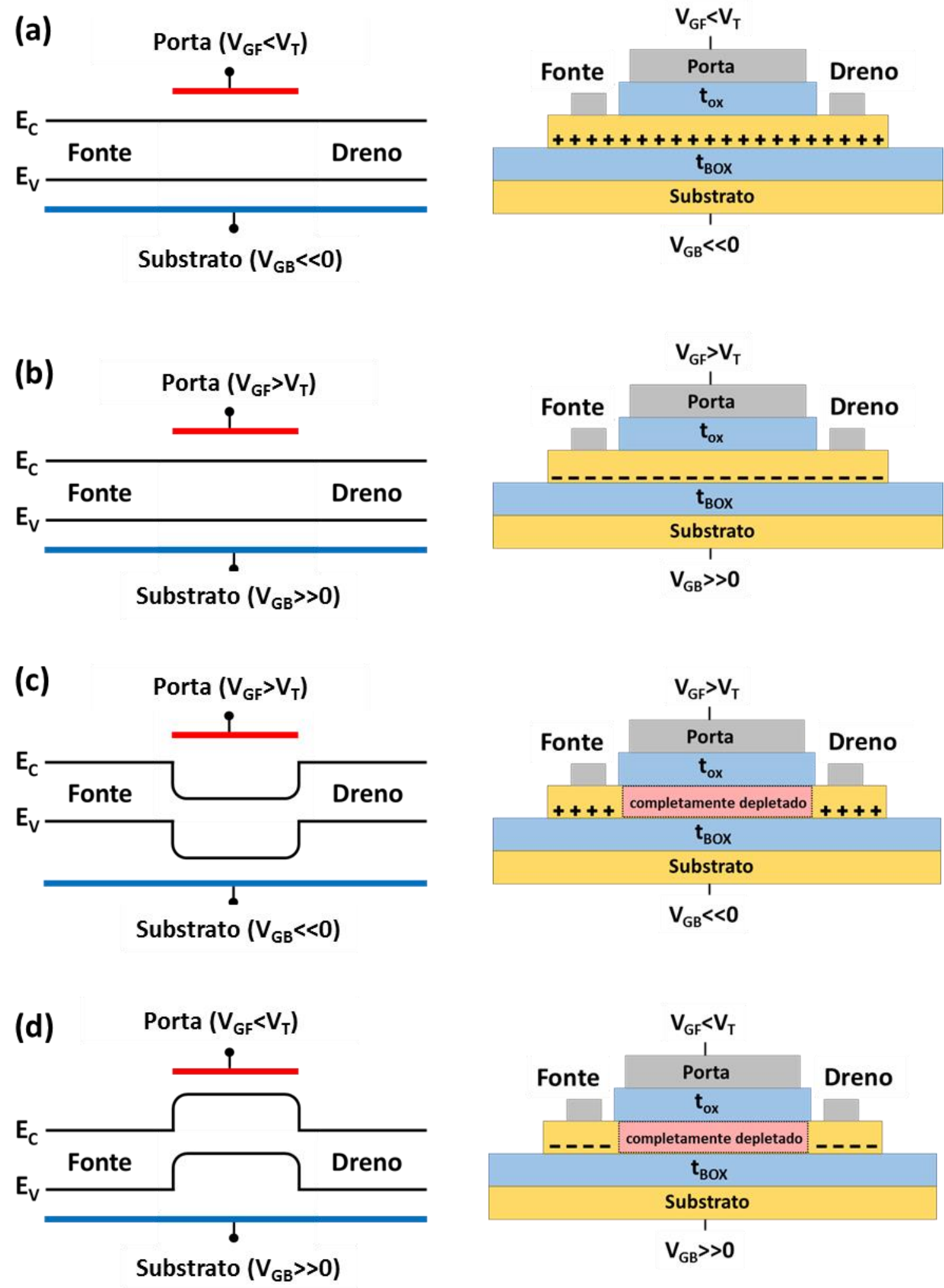

Fonte: adaptado de [31]. 
Na situação em que $\mathrm{V}_{\mathrm{GB}}<<0$ temos o BE SOI MOSFET operando como um transistor tipo $p$, enquanto que na situação em que $V_{G B}>>0$ temos o BE SOI MOSFET operando como um transistor tipo n. Essa característica é um dos grandes diferenciais desse dispositivo, sua flexibilidade quanto ao tipo de transistor, dependendo apenas da polarização do substrato. Dentre outras vantagens podemos citar a simplicidade do processo de fabricação que não exige etapas para dopagem do silício e, consequentemente, evitando problemas para formação de junções em camadas de silício muito finas [32] e problemas relacionados com dopagem flutuante aleatória [47].

A espessura relativamente grande do óxido enterrado exige valores de $V_{G B}$ na ordem de algumas dezenas de Volts para que seja formado a camada de elétrons ou lacunas na segunda interface. Porém este valor de $\mathrm{V}_{\mathrm{GB}}$ pode ser significativamente diminuído se for utilizada lâminas SOI com óxidos enterrados mais finos.

\subsection{Transistor BE SOI fabricado}

O leiaute do circuito integrado onde o BE SOI MOSFET foi fabricado pode ser vista na Figura 3.4. Estão disponíveis transistores com diferentes dimensões de comprimento e largura, entre outras estruturas.

Os transistores utilizados neste trabalho possuem largura de canal de $10 \mu \mathrm{m}$ e comprimento de canal de $50 \mu \mathrm{m}$ e $40 \mu \mathrm{m}$; ou largura de canal de $100 \mu \mathrm{m}$ e comprimento de canal de $100 \mu \mathrm{m}$.

Figura 3.4 - Leiaute do circuito integrado fabricado contendo diversos BE SOI MOSFETs. Em destaque os dispositivos utilizados.

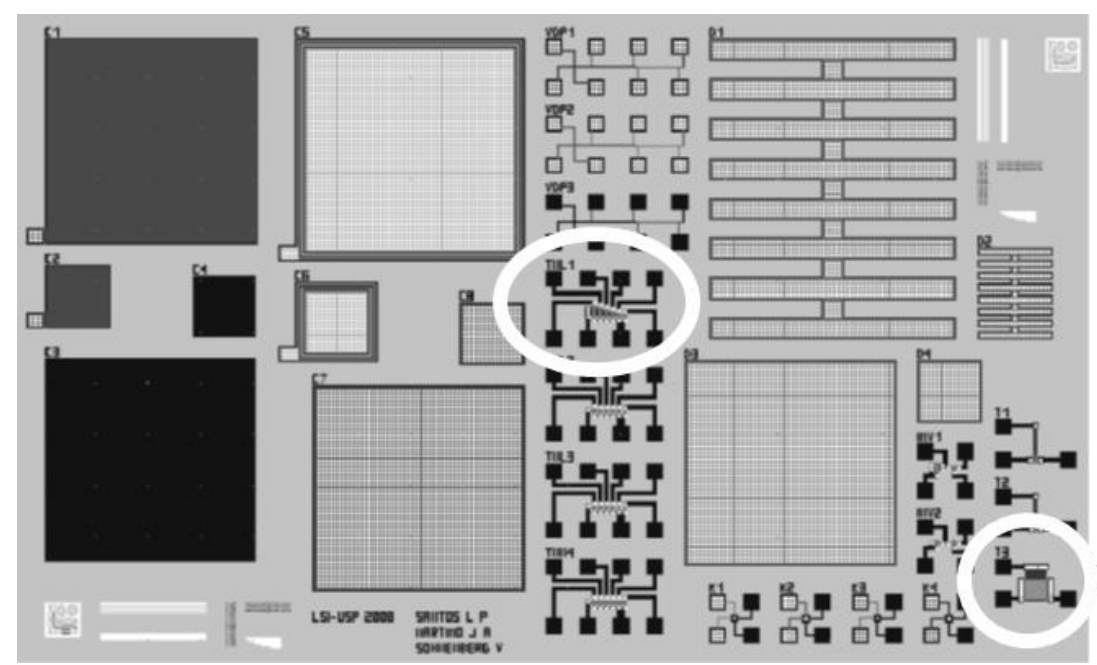

Fonte: [48] 
Duas rodadas de fabricação foram estudadas neste trabalho e serão melhores descritas nos capítulos seguintes. Além das espessuras das camadas de óxido de porta e silício distintas, a principal diferença entre elas é o metal utilizado no contanto com dreno e fonte dos transistores. Na primeira versão do BE SOI MOSFET o metal utilizado foi alumínio, enquanto que na segunda versão foi utilizado o níquel.

\subsection{Medidas experimentais}

As medidas experimentais foram realizadas utilizando os equipamentos HP4156C e o sistema de medidas Agilent B1500, disponíveis no Laboratório de Sistemas Integráveis da Escola Politécnica da USP. Esses equipamentos permitem medidas elétricas e em função de diferentes temperaturas dos transistores.

\subsection{Simulações numéricas}

Além das medidas experimentais, foram realizadas simulações numéricas em duas dimensões dos dispositivos estudados para melhor entendimento dos fenômenos. Foi utilizado o simulador da Synopsys Sentaurus Device [49]. A Figura 3.5 mostra o perfil do dispositivo e a grade de pontos utilizada para as simulações e o anexo $A$ apresenta um exemplo de código utilizado para simular numericamente o $B E$ SOI MOSFET.

Figura 3.5 - Seção transversal do BE SOI MOSFET simulado.

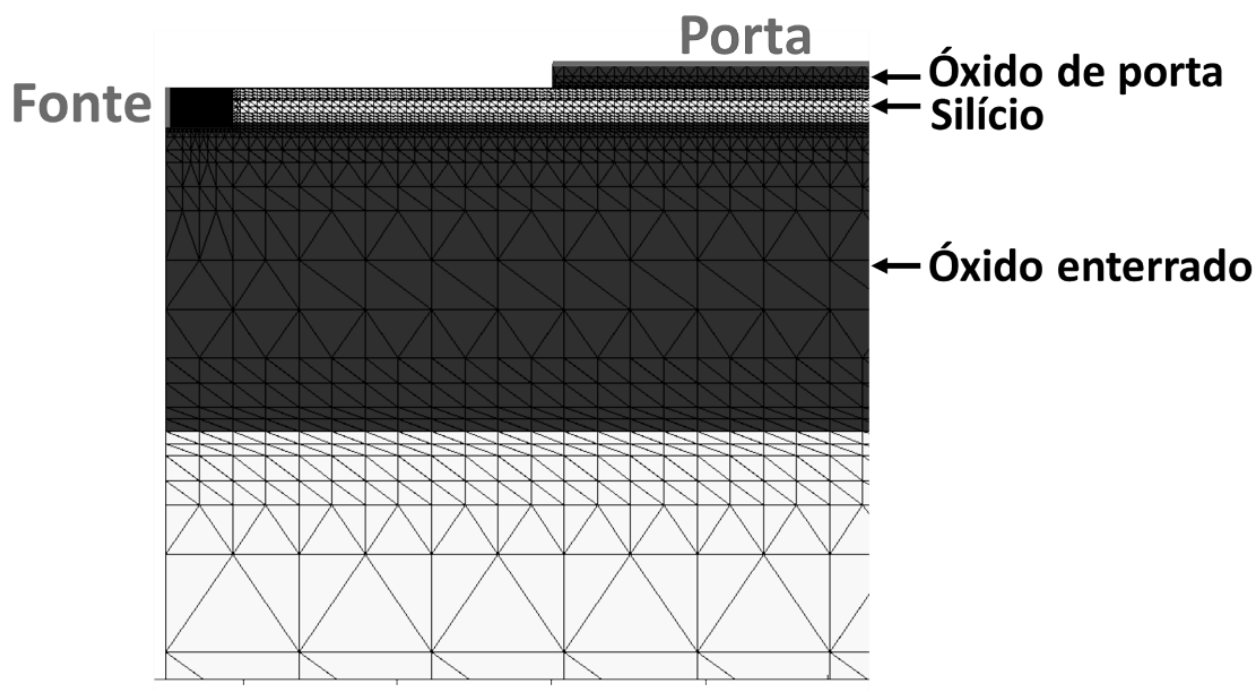

Fonte: Autor. 
Um dos principais modelos utilizados nas simulações foi a definição do tipo de contato entre metal e semicondutor. O Sentaurus Device permite definir contatos tipo Ôhmicos e contatos tipo Schottky, que foram utilizadas para representar as situações em que o metal dos eletrodos de fonte e dreno eram alumínio e níquel respectivamente.

A calibração das simulações foi realizada a partir dos resultados experimentais (Figura 3.6). Os principais modelos e parâmetros que influenciam no BE SOI MOSFET foram adotados, porém algumas simplificações foram adotadas para facilitar a calibração. Dentre os modelos utilizados foram considerados a mobilidade dos portadores dependente da temperatura, recombinação Shockley-Read-Hall e estreitamento da banda proibida. Nas simulações de contatos Schottky foi considerado o modelo não local de tunelamento entre barreiras.

Figura 3.6 - Curvas de corrente de dreno em função da tensão de porta experimentais e simuladas.

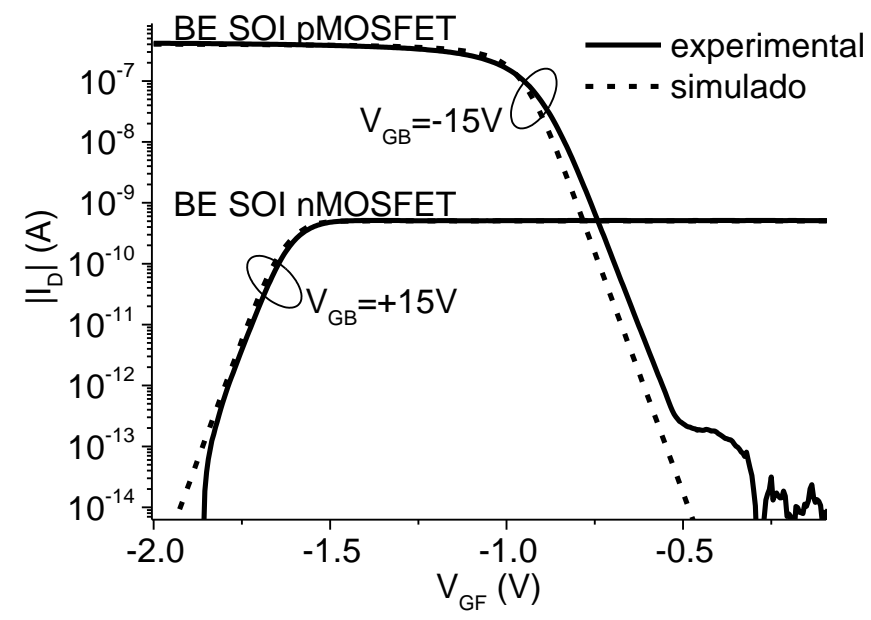

Fonte: Autor.

\subsection{Extração dos parâmetros}

As análises dos dispositivos foram realizadas através de parâmetros básicos extraídos a partir dos resultados experimentais e simulados. A seguir são mostradas como foram extraídos os seguintes parâmetros: tensão de limiar $\left(\mathrm{V}_{\mathrm{T}}\right)$, tranconsutância máxima (gmmáx) e inclinação de sublimar (S) para tensão $V_{D S}$ baixa. 


\subsubsection{Tensão de limiar $\left(V_{T}\right)$}

Foi adotado como valor da tensão de limiar a tensão de porta ( $\left.\mathrm{V}_{\mathrm{GF}}\right)$ correspondente ao máximo da segunda derivada da curva de corrente de dreno em função da tensão de porta $V_{G F}$ [50]. As Figura 3.7 e Figura 3.8 mostram um exemplo de curva de corrente de dreno e sua segunda derivada respectivamente, e a partir desta a tensão de limiar é extraída para um transistor BE SOI pMOSFET.

Figura 3.7 - Exemplo de uma curva lD em função de $V_{G F}$ de um transistor tipo $p$.

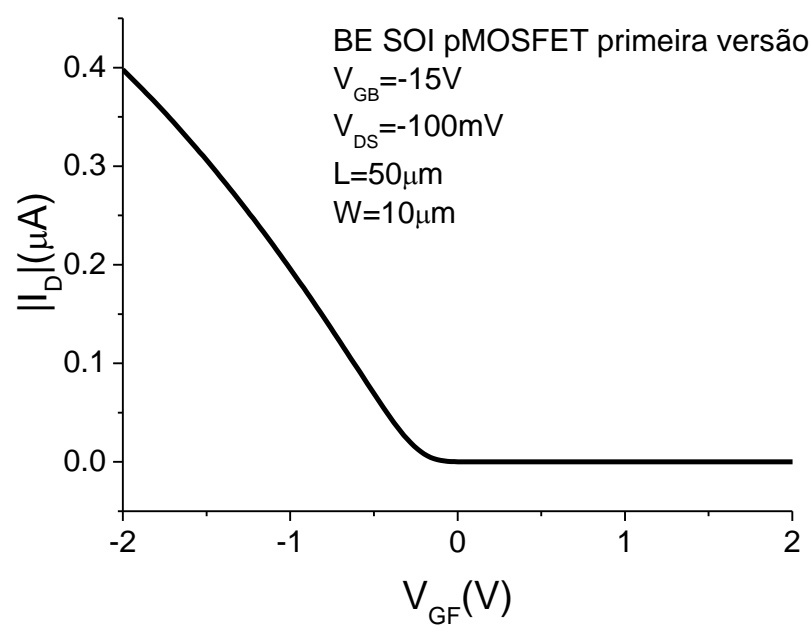

Fonte: Autor.

Figura 3.8 - Exemplo de uma curva $\mathrm{d}^{2} \mathrm{I}_{\mathrm{D}} / \mathrm{dV}_{\mathrm{GF}}{ }^{2}$ em função de $\mathrm{V}_{\mathrm{GF}}$ de um transistor tipo $\mathrm{p}$, mostrando o método de extração da tensão de limiar.

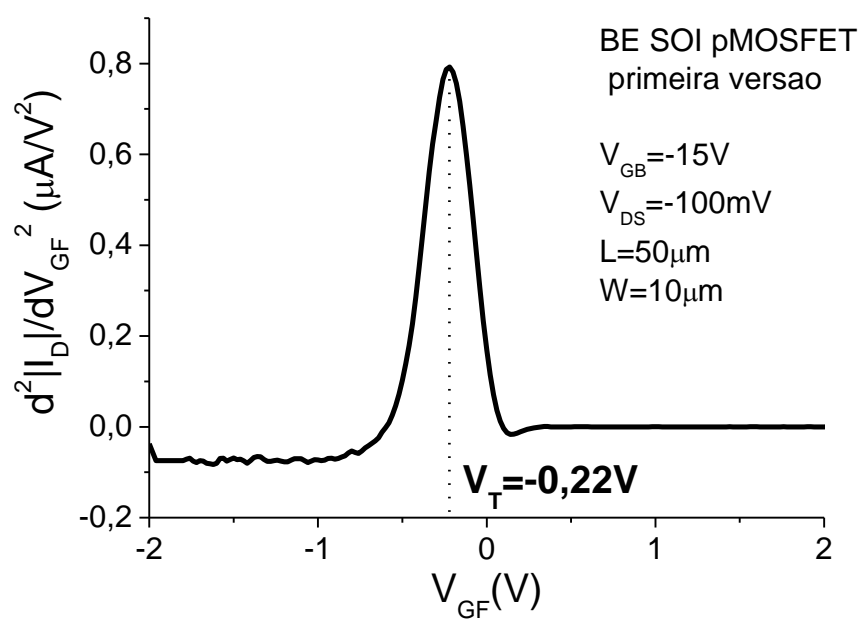

Fonte: Autor. 


\subsubsection{Transcondutância máxima (gmmáx)}

A curva da transcondutância corresponde à primeira derivada da curva da corrente de dreno em função da tensão de porta (VGF). É tomado o valor máximo da curva de transcondutância, no qual temos a situação de máxima mobilidade livre do campo elétrico vertical. A Figura 3.9 mostra a primeira derivada da curva de corrente de dreno em função da tensão de porta (VGF) e o valor da transcondutância máxima extraída.

Figura 3.9 - Exemplo de uma curva $\mathrm{dl}_{\mathrm{D}} / \mathrm{dV}_{\mathrm{GF}}$ em função de $\mathrm{V}_{\mathrm{GF}}$ de um transistor tipo $\mathrm{p}$, mostrando o método de extração da transcondutância máxima.

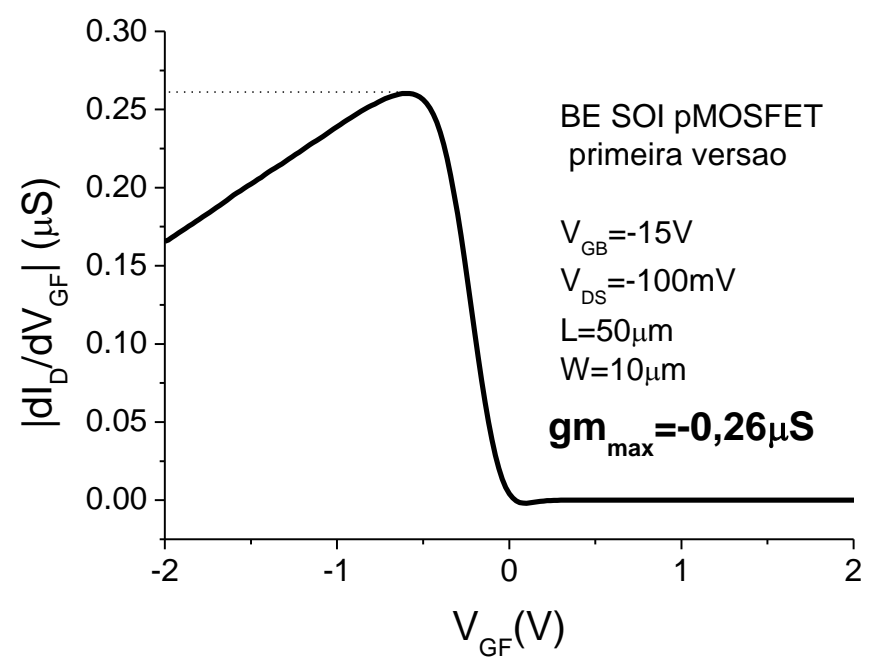

Fonte: Autor.

\subsubsection{Inclinação de sublimiar (S)}

A inclinação de sublimiar é dada pela expressão (2.14) [10].

A partir do inverso da primeira derivada da curva monologarítmica da corrente de dreno em função da tensão de porta é possível encontrar o valor da inclinação de sublimiar como sendo o valor mínimo da curva obtida. 
Figura 3.10 - Exemplo de uma curva $\mathrm{dV}_{\mathrm{GF}} / \mathrm{d}\left(\log \mathrm{l}_{\mathrm{D}}\right)$ em função de $\mathrm{V}_{\mathrm{GF}}$ de um transistor tipo $\mathrm{p}$, mostrando o método de extração da inclinação de sublimiar mínima.

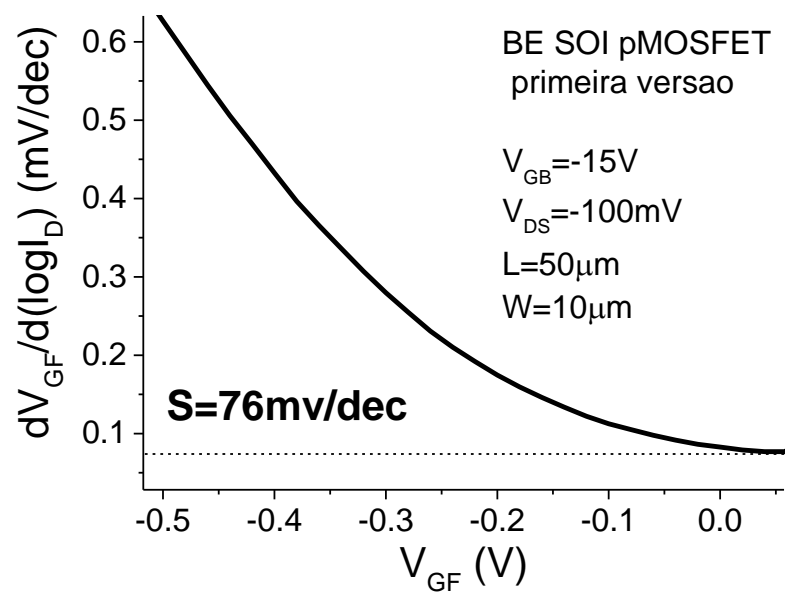

Fonte: Autor.

\subsubsection{Fator de degradação da mobilidade com a temperatura (c)}

O coeficiente de degradação da mobilidade com a temperatura foi extraído a partir da expressão (3.1) [38].

$$
\frac{\mathrm{gm}_{\text {máx1 }}}{\mathrm{gm}_{\text {máx2 }}}=\frac{\mu_{1}}{\mu_{2}}=\left(\frac{\mathrm{T}_{2}}{\mathrm{~T}_{1}}\right)^{\mathrm{c}}
$$

Onde gmmáx1 e $\mu_{1}$ são a transcondutância máxima e a mobilidade dos portadores para a temperatura de referência $T_{1}$, respectivamente, e gmmáx2 e $\mu_{2}$ são a transcondutância máxima e a mobilidade dos portadores para a temperatura de $\mathrm{T}_{2}$.

A partir das máximas transcondutâncias para diversas temperaturas, adotouse a temperatura ambiente como referência. Utilizou-se a expressão (3.1) para calcular o valor do coeficiente $\mathrm{C}_{\mathrm{Ti}}$ para as demais temperaturas $T_{i}$ em relação à temperatura de referência. Neste trabalho, foi considerado como o valor para o coeficiente de degradação da mobilidade c a média dos valores calculados ст.

\subsubsection{Fator de corpo $(n)$}

Ao contrário de um transistor SOI MOSFET convencional no qual a corrente passa pela primeira interface, no BE SOI MOSFET a corrente de dreno circula pela 
segunda interface. $O$ fator de corpo pode ser calculado a partir da relação entre a capacitâncias equivalentes vistas a partir da segunda interface, onde a corrente circula predominantemente, conforme mostrado na Figura 3.11.

Figura 3.11 - Modelo de capacitâncias representando o acoplamento entre a porta e o substrato no BE SOI MOSFET.

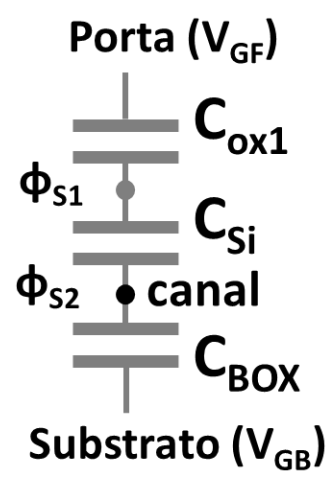

Fonte: Autor.

Desta forma o fator capacitivo $\alpha$ correspondente à variação da tensão de limiar $\mathrm{V}_{\mathrm{T}}$ em relação a tensão de substrato $\mathrm{V}_{\mathrm{GB}}$ pode ser calculado pela equação (3.2) no caso do BE SOI MOSFET, ao invés da equação (2.18). O denominador da expressão corresponde a associação em série da capacitância do óxido de porta e da capacitância do canal.

$$
\frac{\mathrm{dV}_{\mathrm{T}}}{\mathrm{dV}_{\mathrm{GB}}}=-\frac{\mathrm{C}_{\mathrm{BOX}}}{\left(\frac{1}{\mathrm{C}_{\mathrm{OX}}}+\frac{1}{\mathrm{C}_{\mathrm{Si}}}\right)^{-1}}=-\alpha
$$

E o fator de corpo pode ser calculado como $n=1+\alpha$. 


\section{BE SOI MOSFET primeira versão}

Neste capítulo é apresentado a primeira versão do BE SOI MOSFET que possui espessura de óxido de porta tox=15nm, espessura do silício tsi $=23 \mathrm{~nm}$ e espessura do óxido enterrado tBox $=200 \mathrm{~nm}$, conforme mostrado na Figura 4.1. O transistor utilizado possui comprimento $L=50 \mu \mathrm{m}$ e a largura $\mathrm{W}=10 \mu \mathrm{m}$. Uma característica relevante que afetará diretamente os resultados obtidos é o material de contato de fonte e dreno utilizado, o alumínio não dopado, e a etapa de sinterização utilizada. A Figura 4.2 mostra uma cascata de transistores fabricados no LSI com transistores de diferentes comprimentos.

Figura 4.1 - Perfil esquemático da primeira versão fabricada do BE SOI MOSFET.

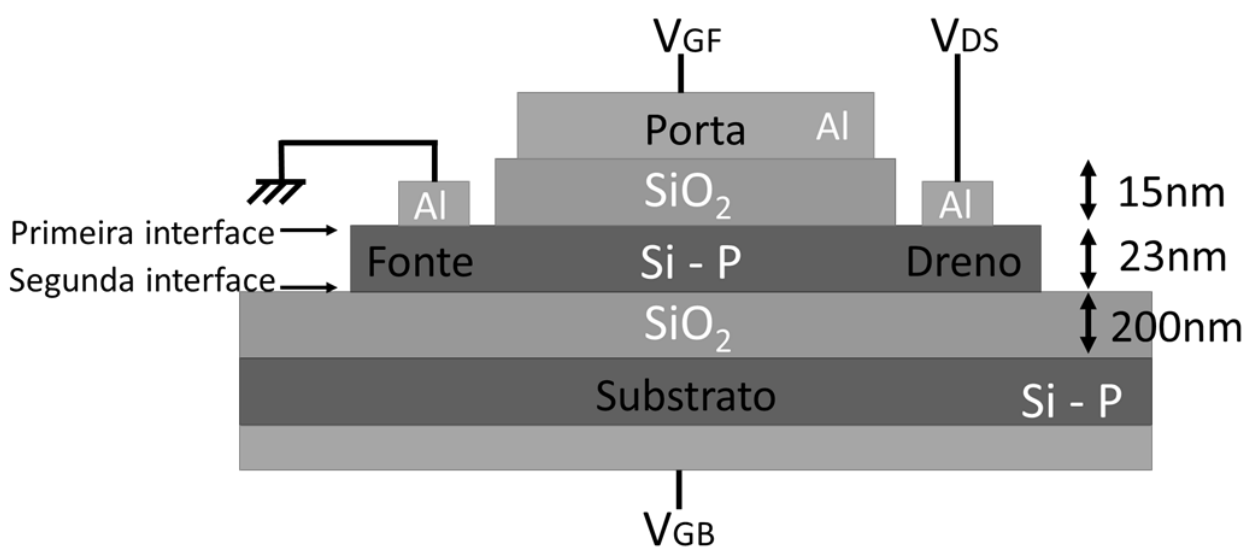

Fonte: Autor.

Figura 4.2 - Foto da primeira versão do transistor BE SOI MOSFET fabricado.

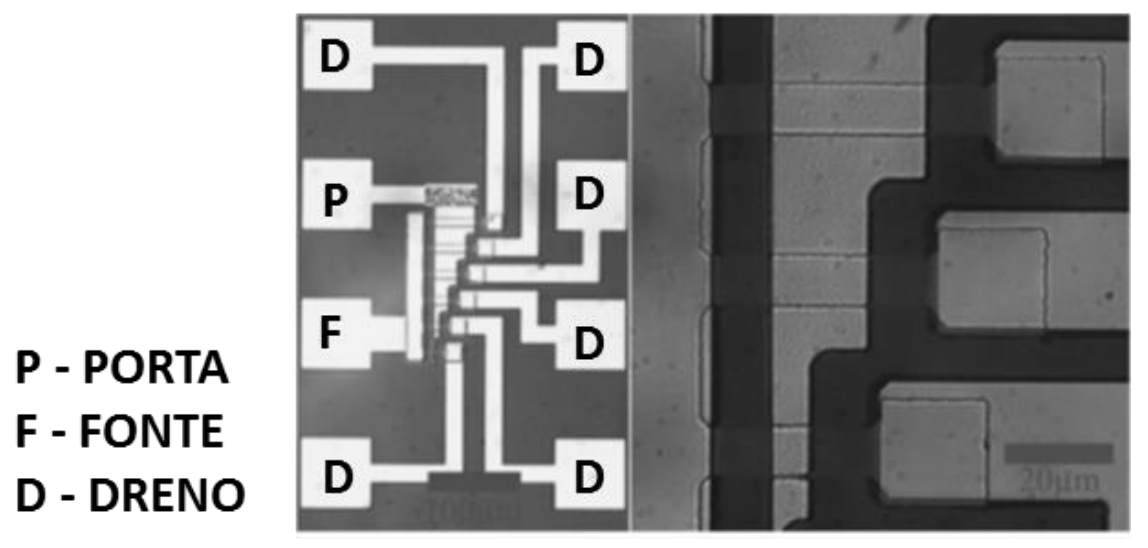

Fonte: [31]. 


\subsection{Resultados e análises}

Os resultados das medidas elétricas experimentais são encontrados em [31] e serão apresentados em seguida. Finalmente, serão mostrados os resultados das medidas elétricas experimentais realizadas em função da temperatura [51].

\subsubsection{Medidas em temperatura ambiente}

A curva de transferência típica do transistor BE SOI pMOSFET pode ser encontrada na Figura 4.3. Foram realizadas medidas alterando o valor da tensão de substrato $\mathrm{V}_{\mathrm{GB}}$ variando de $-15 \mathrm{~V}$ à $-35 \mathrm{~V}$ e com isso é possível notar que quanto maior o valor de $\mathrm{V}_{\mathrm{GB}}$, maior é a corrente de dreno para o mesmo $\mathrm{V}_{\mathrm{GF}}$. Isso ocorre principalmente devido ao deslocamento do valor da tensão de limiar $\mathrm{V}_{\mathrm{T}}$ em função de $V_{G B}$. Quanto maior a $V_{G B}$, maior é o campo elétrico vertical gerado pelo substrato e, portanto, maior deve ser o valor de $\mathrm{V}_{\mathrm{GF}}$ para contrapor esse campo elétrico e depletar a camada de silício.

Figura 4.3 - Curvas de transferência ID em função de $V_{G F}$ medidas experimentalmente para diferentes valores de $\mathrm{V}_{\mathrm{GB}}$.

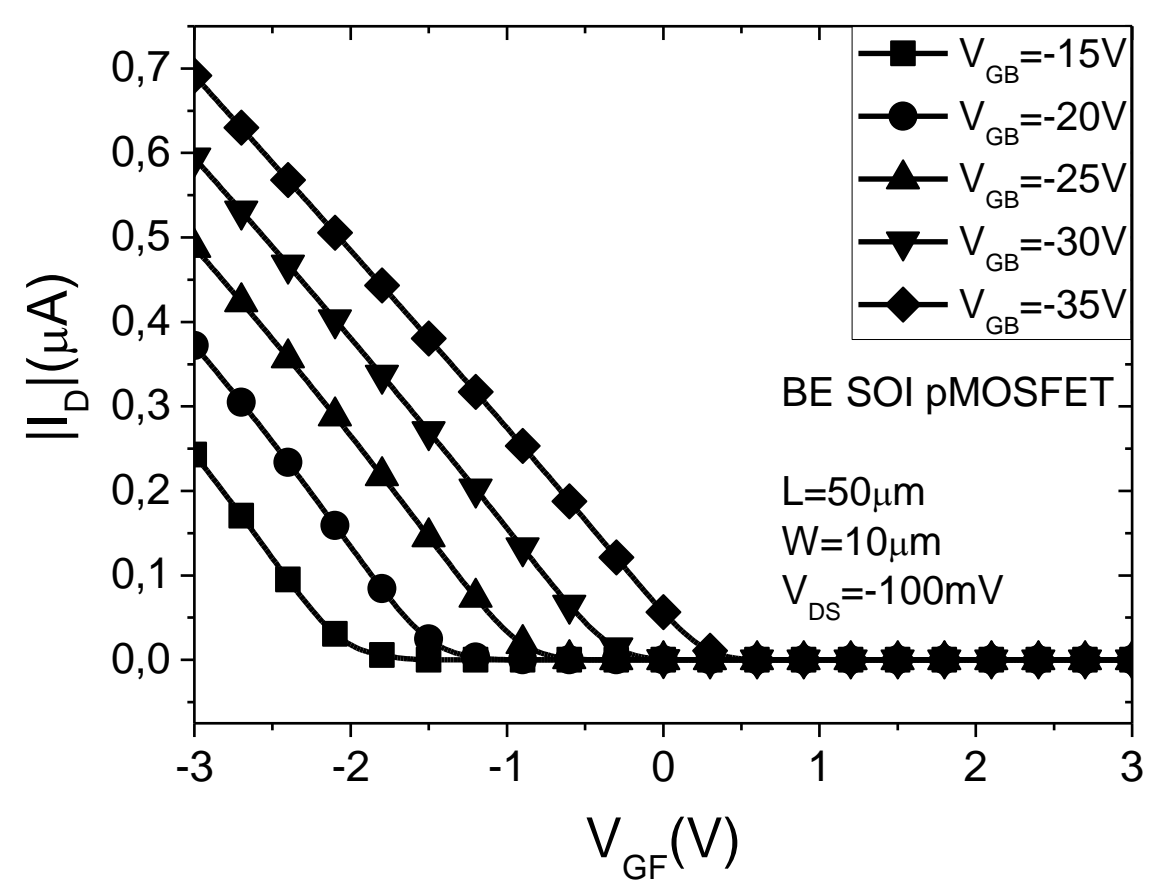

Fonte: Autor. 


\subsubsection{Medidas em altas temperaturas e ZTC}

Foram realizadas medidas experimentais elétricas do transistor BE SOI pMOSFET para avaliar a influência da temperatura no seu funcionamento. As medidas de corrente de dreno em função de $V_{G F}$ do transistor de comprimento $L=50 \mu \mathrm{m}$ e largura $\mathrm{W}=10 \mu \mathrm{m}$ variaram de $25^{\circ} \mathrm{C}$ a $125^{\circ} \mathrm{C}$ para tensões $V_{G B}$ variando de $-15 \mathrm{~V}$ a $35 \mathrm{~V}$. Nessa etapa foi tomado o cuidado para evitar danificar os dispositivos estudados, uma vez que o efeito da temperatura em conjunto com os altos valores de tensão aplicados devido à espessa camada de óxido enterrado facilitava o rompimento da mesma, danificando permanentemente os dispositivos. Portanto as medidas com $V_{G B}=-35 \mathrm{~V}$ e temperaturas de $100^{\circ} \mathrm{C}$ e $125^{\circ} \mathrm{C}$ e $V_{G B}=-30 \mathrm{~V}$ e temperatura de $125^{\circ} \mathrm{C}$ não foram realizadas para evitar esses problemas.

Figura 4.4 - Curvas de transferência ID em função de $V_{G F}$ medidas experimentalmente para diferentes temperaturas em escala linear.

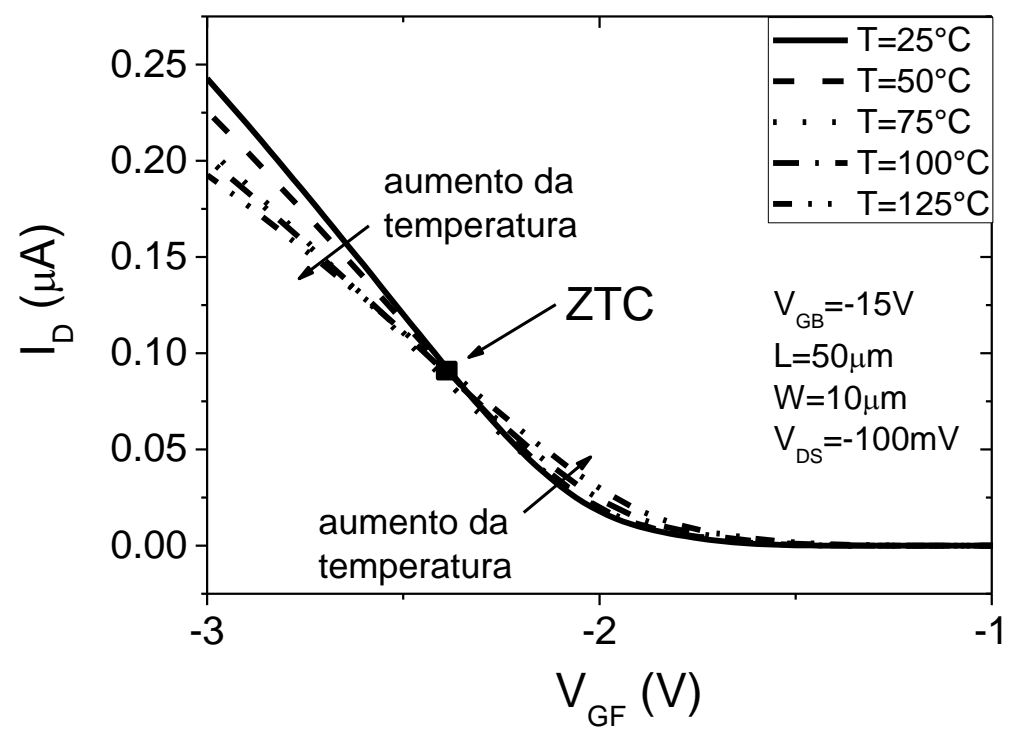

Fonte: Autor.

A Figura 4.4 mostra as curvas de transferência em escala linear para tensão de substrato $\mathrm{V}_{\mathrm{GB}}=-15 \mathrm{~V}$ e diferentes temperaturas e a Figura 4.5 mostra o mesmo resultado em escala logarítmica. É possível notar a presença de uma região invariante com a temperatura ZTC neste dispositivo no qual há uma diminuição da corrente de dreno em função da temperatura para $V_{G F}<V_{Z T C}$ e há um aumento da corrente de dreno em função da temperatura para $V_{G F}>V_{Z T C}$. A presença do $Z T C$ também ocorre para os demais valores de $V_{G B}$ utilizados nas medidas. 
Figura 4.5 - Curvas de transferência ID em função de $V_{G F}$ medidas experimentalmente para diferentes temperaturas em escala logarítmica.

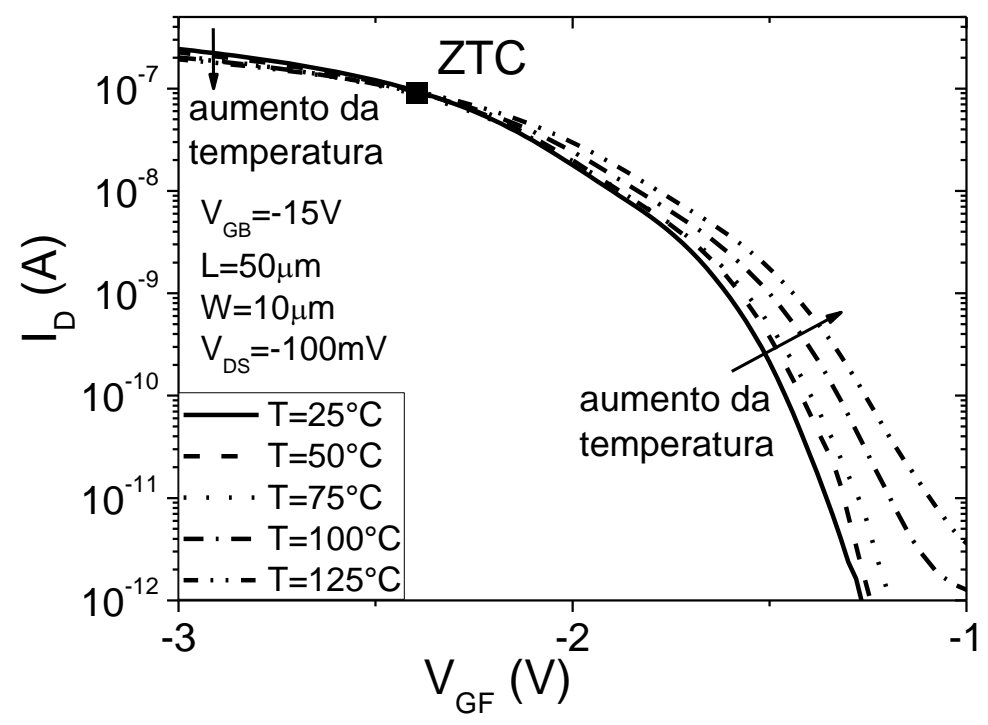

Fonte: Autor.

A tensão de limiar foi extraída para todas as medidas realizadas pelo método da segunda derivada descrito anteriormente e pode ser visto na Figura 4.6. É possível notar um aumento de $\mathrm{V}_{\mathrm{T}}$ em função da temperatura devido a variação do potencial de Fermi $\phi F$.

Realizando ajustes linear nos dados levantados, foi possível encontrar os seguintes valores para variação de tensão de limiar em função da temperatura $(\mathrm{dV} / \mathrm{dT}): 1,52 \mathrm{mV} /{ }^{\circ} \mathrm{C}$ para $\mathrm{V}_{\mathrm{GB}}=-15 \mathrm{~V} ; 1,2 \mathrm{mV} /{ }^{\circ} \mathrm{C}$ para $\mathrm{V}_{\mathrm{GB}}=-20 \mathrm{~V} ; 1,36$ para $\mathrm{V}_{\mathrm{GB}}=-25 \mathrm{~V}$; $1,04 \mathrm{mV} /{ }^{\circ} \mathrm{C}$ para $V_{\mathrm{GB}}=-30 \mathrm{~V}$ e $1,2 \mathrm{mV} /{ }^{\circ} \mathrm{C}$ para $\mathrm{V}_{\mathrm{GB}}=-35 \mathrm{~V}$. Tais valores para variação $\mathrm{da}$ tensão de limiar em função da temperatura (que chegaram até $1,52 \mathrm{mV} /{ }^{\circ} \mathrm{C}$ ) são maiores que valores típicos observados em transistores SOI completamente depletados convencionais, cujo valor depende apenas do potencial de Fermi é em torno de $0,7 \mathrm{mV} /{ }^{\circ} \mathrm{C}[10]$. Esta diferença ocorre devido ao princípio de operação distinto do BE SOI MOSFET, no qual a corrente que passa através da segunda interface depende do acoplamento eletrostático entre os dois eletrodos de porta $V_{G F}$ e $V_{G B}$. No BE SOI MOSFET a tensão de limiar ocorre quando a segunda interface da camada de silício está depletada, diferentemente de um transistor SOI completamente depletado no qual a tensão de limiar ocorre quando a primeira interface da camada de silício atinge a inversão. 
Figura 4.6 - Tensão de limiar em função da temperatura para diferentes valores de $V_{G B}$.

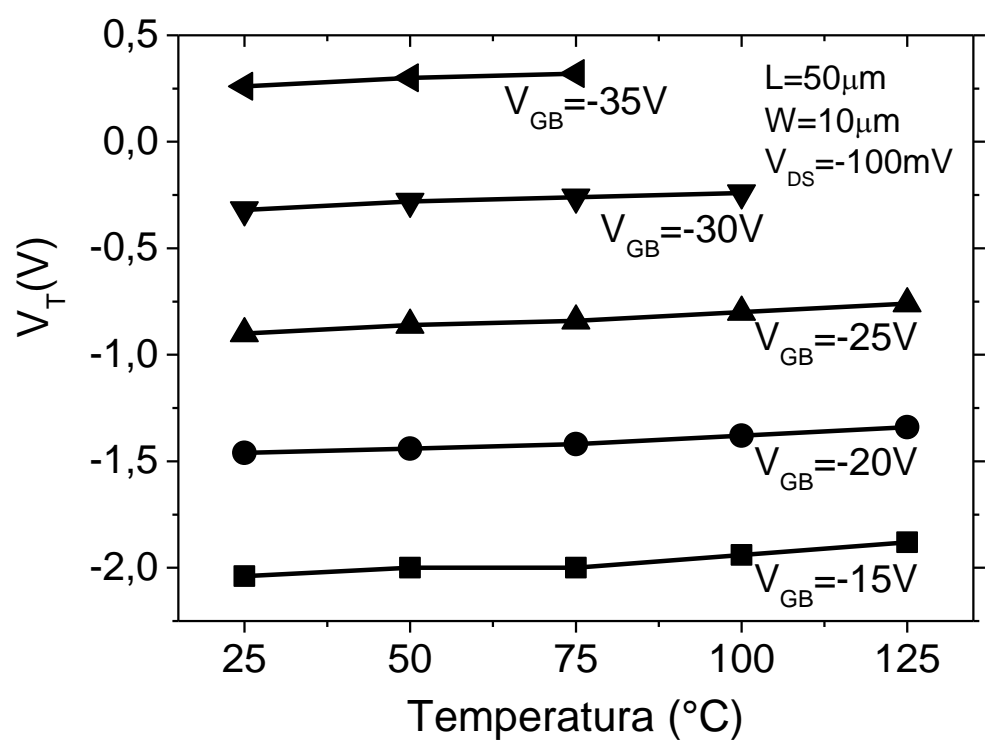

Fonte: Autor.

Os mesmos dados das tensões de limiar da Figura 4.6 são mostrados novamente na Figura 4.7, porém agora em função da tensão de substrato $V_{G B}$. $A$ tensão $V_{G F}$ necessária para interromper a condução de corrente formada na segunda interface aumenta conforme $\mathrm{V}_{\mathrm{GB}}$ se torna mais negativo (no caso do transistor BE SOI pMOSFET).

$A$ variação da tensão de limiar em relação a $\bigvee_{G B}$ foi calculada conforme descrito anteriormente (equação (3.2)) utilizando as espessuras deste dispositivo e foi obtido o valor para o coeficiente $\alpha=0,112$. Realizando ajustes linear das curvas obtidas na Figura 4.7 para as diferentes temperaturas obtemos os seguintes valores para a variação da tensão de limiar em relação a tesão de substrato $\left(\mathrm{dV}_{\mathrm{T}} / \mathrm{dV} \mathrm{GB}\right)$ : $-0,1148$ para $\mathrm{T}=25^{\circ} \mathrm{C} ;-0,1152$ para $\mathrm{T}=50^{\circ} \mathrm{C} ;-0,116$ para $\mathrm{T}=75^{\circ} \mathrm{C} ;-0,1136$ para $\mathrm{T}=100^{\circ} \mathrm{C}$ e $-0,112$ para $\mathrm{T}=125^{\circ} \mathrm{C}$. Portanto o fator a se mostrou independente da temperatura e os valores experimentais obtidos se aproximaram do valor teórico calculado, comprovando o princípio de funcionamento do BE SOI MOSFET e que a corrente circula principalmente pela segunda interface. 
Figura 4.7 - Tensão de limiar em função da polarização $V_{G B}$ para diferentes temperaturas.

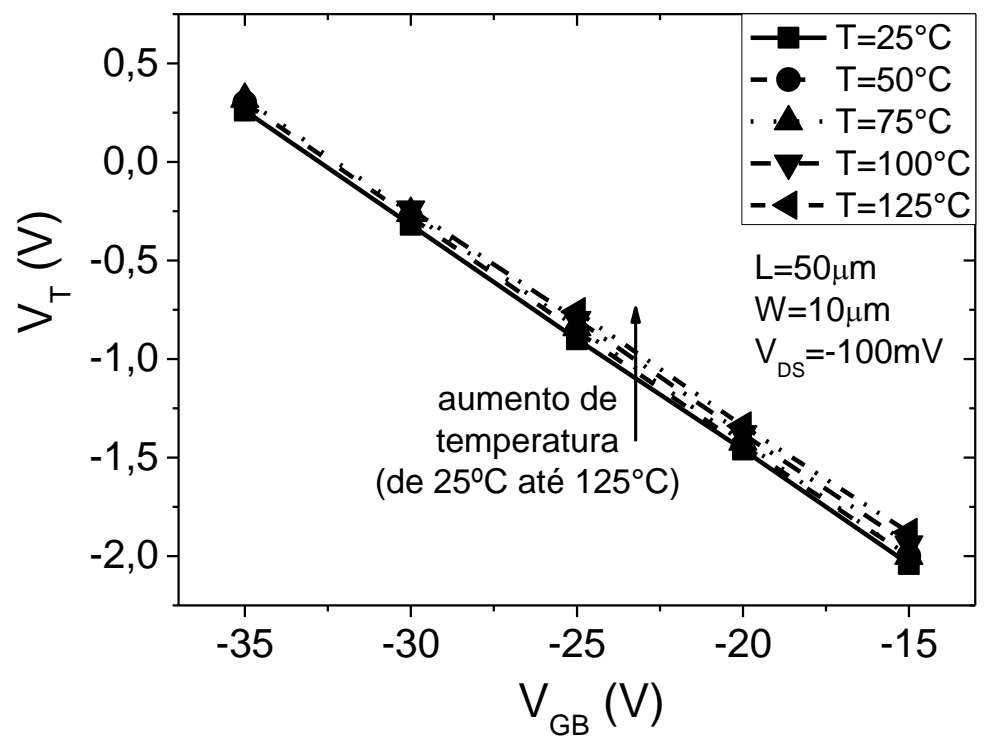

Fonte: Autor.

O valor máximo da transcondutância foi extraído conforme mostrado na Figura 4.8. O resultado decrescente para esse parâmetro em função da temperatura evidencia que a mobilidade dos portadores está sendo degradada para temperaturas mais elevadas. Apesar de que a mobilidade não foi extraída diretamente, podemos chegar nessa conclusão uma vez que a transcondutância máxima do dispositivo é diretamente proporcional à mobilidade dos portadores.

Figura 4.8 - Transcondutância máxima em função da temperatura para diferentes valores de $\mathrm{V}_{\mathrm{GB}}$.

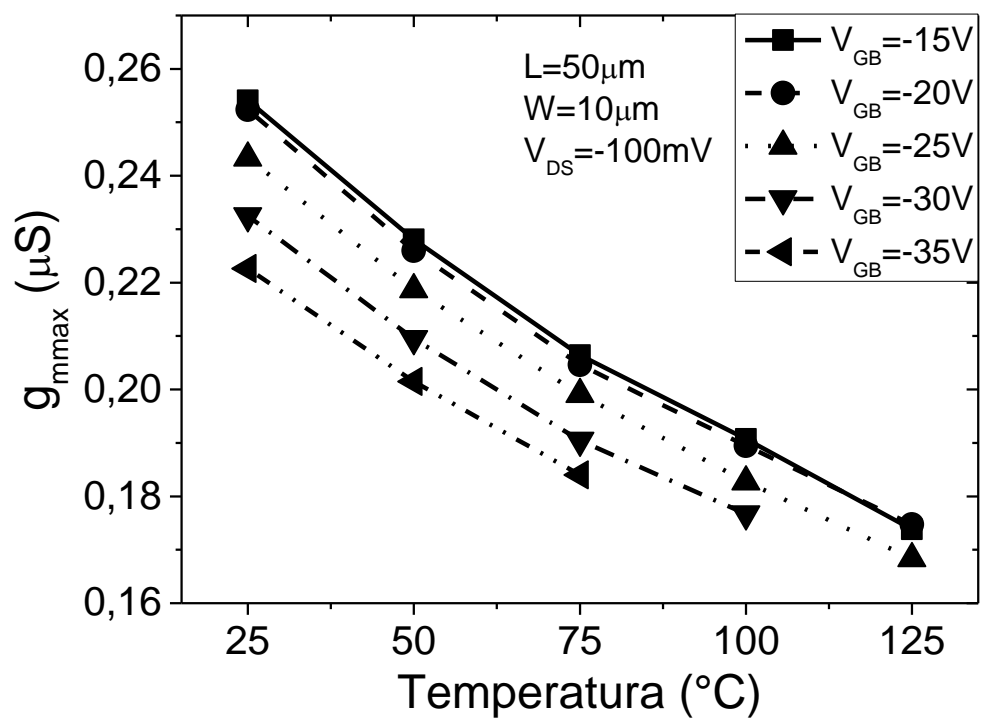

Fonte: Autor. 
A Figura 4.9 mostra a inclinação de sublimiar S em função da temperatura. Há uma degradação deste parâmetro para temperaturas elevadas, mais acentuada que em transistores SOI MOSFET convencionais. Normalmente a variação de $\mathrm{S}$ em função da temperatura é proporcional à kT/q e nesse caso ela é maior [10], sendo k a constante de Boltzmann, T a temperatura em Kelvin e q a carga elementar.

Figura 4.9 - Inclinação de sublimiar em função da temperatura para diferentes valores de $\mathrm{V}_{\mathrm{GB}}$.

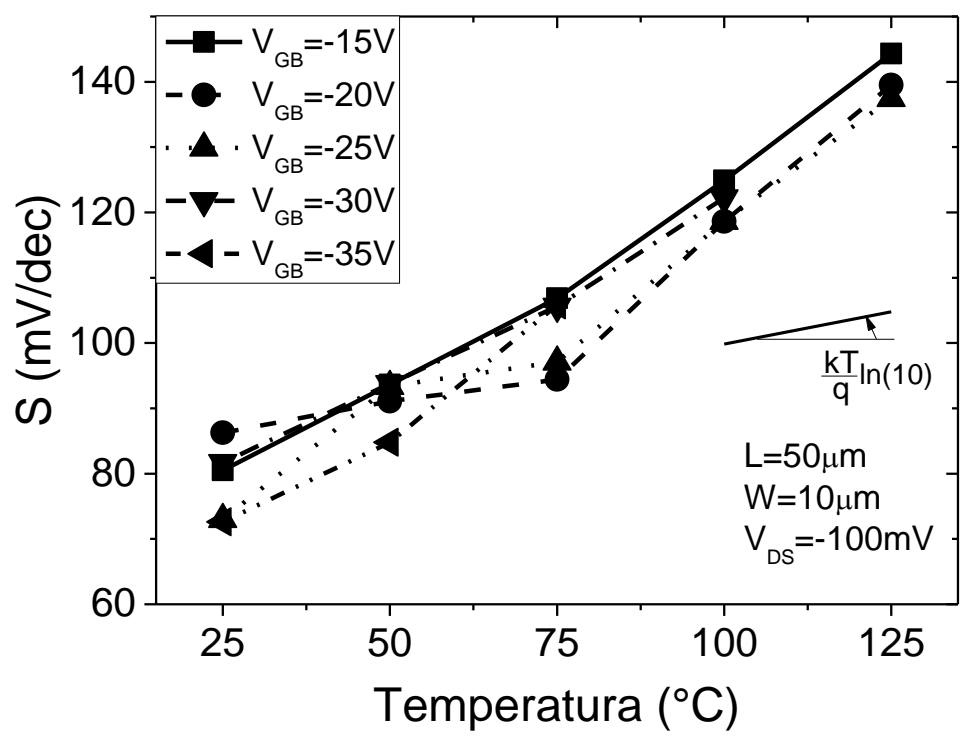

Fonte: Autor.

\subsubsection{Determinação do ponto invariante com a temperatura ZTC na região linear}

Conforme visto anteriormente nas Figura 4.4 e Figura 4.5, o BE SOI pMOSFET da primeira versão apresenta um ponto de polarização invariante com a temperatura. Esse resultado pode ser explicado através das análises dos parâmetros extraídos. Dentre eles, foi visto que há uma variação da tensão de limiar e que como consequência a corrente de dreno aumenta em função da temperatura. Por outro lado, a análise da transcondutância máxima do dispositivo mostrou que há uma degradação da mobilidade dos portadores para temperaturas elevadas. Como resultado da competição entre esses dois fenômenos que ocorrem com o aumento da temperatura, temos a presença do ponto invariante com a temperatura ZTC.

A fim de se estudar essa condição de polarização, foi utilizado o modelo descrito anteriormente para ZTC na região linear de operação do transistor. 
O fator de degradação da mobilidade c foi calculado para cada tensão de substrato utilizando a expressão (3.1) descrita anteriormente e adotando a temperatura de $25^{\circ} \mathrm{C}$ como referência. Os valores obtidos foram: 1,32 para $V_{G B}=-15 V$; 1,32 para $V_{G B}=-20 V ; 1,29$ para $V_{G B}=-25 V ; 1,27$ para $V_{G B}=-30 V$ e 1,23 para $V_{G B}=-35 V$.

A tensão de porta $V_{G F}$ correspondente ao ponto ZTC foi calculado utilizando o modelo linear (equação (2.32)). Nessa expressão foram adotados os valores obtidos anteriormente para o fator $\mathrm{c}$, o fator de corpo $\mathrm{n}$ e a variação da tensão de limiar em função da temperatura $\mathrm{dV} \mathrm{T} / \mathrm{dT}$.

A Figura 4.10 mostra a comparação dos resultados experimentais da tensão correspondente ao ponto ZTC (VZTC) com o teórico calculado através do modelo linear.

Figura 4.10 - Comparação dos valores de $V_{Z T C}$ obtidos experimentalmente e através do modelo.

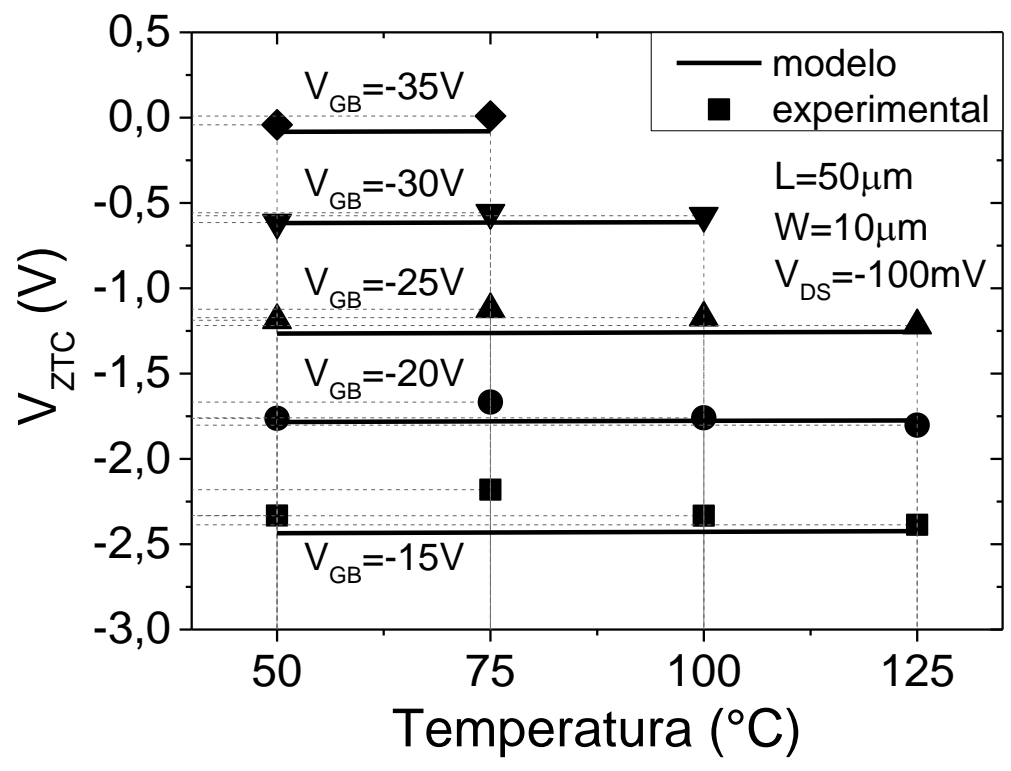

Fonte: Autor.

É possível observar um aumento do valor de VzTC quanto menor é o valor de $V_{G B}$ para o caso de um BE SOI pMOSFET devido a maior tensão de porta $V_{G F}$ necessária para depletar a camada de silício.

Apesar do modelo para cálculo de VZTC não ter sido desenvolvido para o transistor BE SOI MOSFET, a comparação entre os resultados experimentais e os modelados mostrou uma pequena diferença entre eles. A máxima diferença foi de $0,25 \mathrm{~V}$, correspondendo a um erro de aproximadamente $11,5 \%$. 


\subsection{BE SOI nMOSFET}

Até o momento foram apresentados apenas resultados referentes ao transistor BE SOI pMOSFET. Seguindo a explicação dada para o princípio de funcionamento dos transistores BE SOI MOSFET, caso a tensão de substrato $V_{G B}$ fosse positiva, seria criada uma camada de elétrons na segunda interface do filme de silício e teríamos um transistor tipo $\mathrm{n}$ com funcionamento análogo ao BE SOI pMOSFET.

Entretanto foi notado nesta primeira versão do BE SOI MOSFET que o transistor não apresentava a curva característica de transferência quando utilizado um valor de $\mathrm{V}_{\mathrm{GB}}>0$. Foi constatado que a camada de elétrons na segunda interface não era formada neste caso devido à natureza do metal utilizado como eletrodos de fonte e dreno e, portanto, não havia formação do transistor.

O metal de contato com fonte e dreno é o alumínio, cuja função trabalho vale cerca de 4,1eV e cuja a tendência é de formação de uma junção ôhmica com o silício após etapa térmica. Este fato e a baixa dopagem do silício impedem a formação da camada de elétrons na segunda interface. Em um transistor MOSFET convencional, as junções de fonte e dreno são responsáveis por fornecer as cargas de inversão que formam o canal, porém no caso do BE SOI MOSFET, a ausência de junções faz com que o mesmo não ocorra.

Portanto a primeira versão fabricada do BE SOI MOSFET não apresentou funcionamento adequado como transistor tipo $\mathrm{n}$. 


\section{BE SOI MOSFET segunda versão}

Neste capítulo é apresentado a segunda versão do BE SOI MOSFET, fabricada sobre uma lâmina SOI com espessura do óxido enterrado tBOx=200nm. O óxido de porta possui espessura tox $=10 \mathrm{~nm}$ e espessura do silício tsi $=10 \mathrm{~nm}$, conforme mostrado na Figura 5.1. Foram utilizados transistores com comprimento $L=40 \mu m$ e largura $\mathrm{W}=10 \mu \mathrm{m}$ e transistores com comprimento $\mathrm{L}=100 \mu \mathrm{m}$ e largura $\mathrm{W}=100 \mu \mathrm{m}$. A Figura 5.2 mostra a fotografia de um transistor fabricado.

Figura 5.1 - Perfil esquemático da segunda versão fabricada do BE SOI MOSFET.

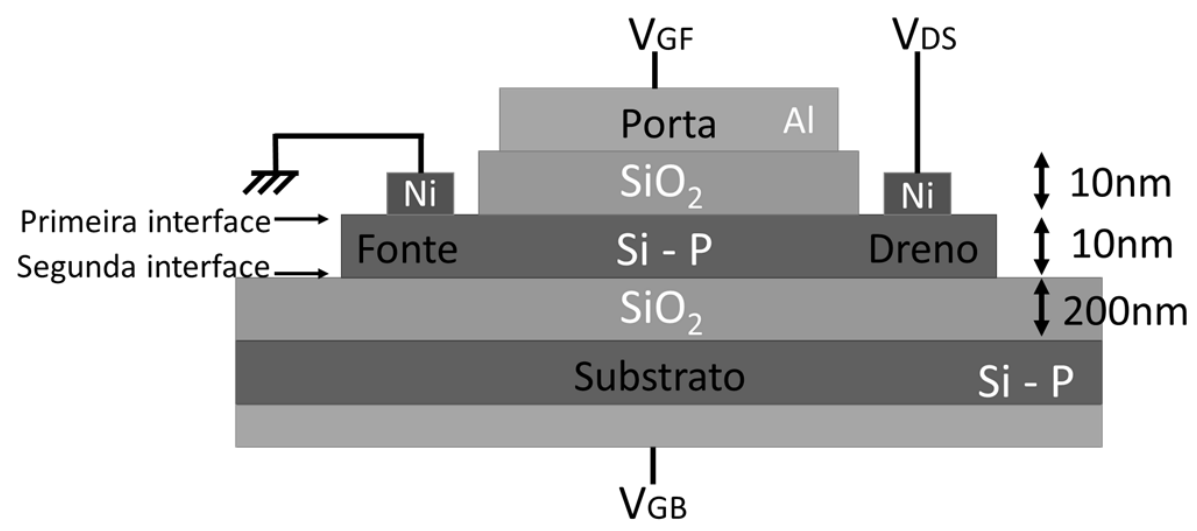

Fonte: Autor.

Figura 5.2 - Foto da primeira versão do transistor BE SOI MOSFET fabricado.

P - PORTA

F - FONTE

D - DRENO

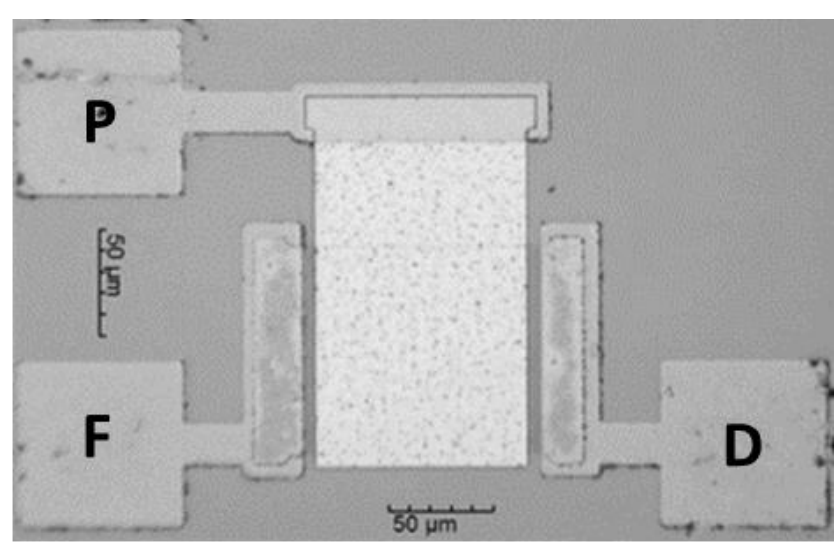

Fonte: Autor.

Diferente da primeira versão, os contatos de fonte e dreno foram construídos utilizando níquel como metal ao invés do alumínio. Após a etapa de sinterização, há formação de uma liga de siliceto de níquel, cuja tendência é a de formar um contato 
tipo Schottky com o silício. A utilização do siliceto de níquel, cuja função trabalho vale entre $4,7 \mathrm{eV}$ e $4,8 \mathrm{eV}$, cria barreiras de potencial de tamanhos simétricos tanto para lacunas quanto para elétrons [22]. Isto possibilita a formação da camada de lacunas caso $\mathrm{V}_{\mathrm{GB}}<0$ ou a formação da camada de elétrons caso $\mathrm{V}_{\mathrm{GB}}>0$ na segunda interface, o que não ocorria na primeira versão do BE SOI MOSFET pois os contatos de alumínio propiciavam barreiras de potencial para elétrons maior do que para lacunas.

$\mathrm{Na}$ segunda versão fabricado do BE SOI MOSFET foi possível alcançar transistores flexíveis quanto ao seu tipo. Dependendo apenas da polarização do substrato podemos obter tanto transistor tipo $\mathrm{n}$ quanto transistores tipo $\mathrm{p}$ com $\mathrm{o}$ mesmo dispositivo.

\subsection{Resultados e análises}

Foram realizadas medidas elétricas experimentais para diversos valores de $V_{G B}$, a fim de se comprovar o correto funcionamento do transistor. A Figura 5.3 mostra o resultado obtido para o BE SOI pMOSFET e a Figura 5.4 o resultado para o BE SOI nMOSFET.

Figura 5.3 - Curvas de transferência ID em função de $V_{G F}$ medidas experimentalmente para diferentes valores de $\mathrm{V}_{\mathrm{GB}}$, mostrando o funcionamento do $\mathrm{BE} S \mathrm{SOI}$ pMOSFET.

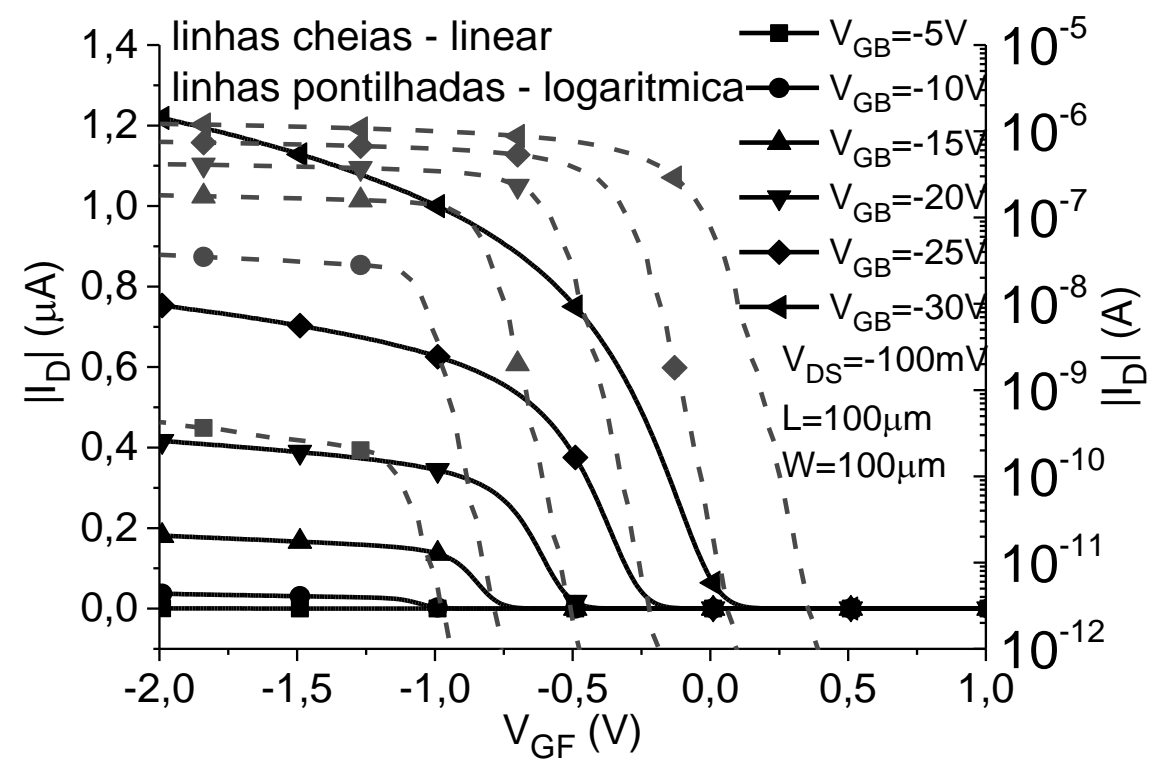

Fonte: Autor. 
Figura 5.4 - Curvas de transferência $I_{D}$ em função de $V_{G F}$ medidas experimentalmente para diferentes valores de $\mathrm{V}_{\mathrm{GB}}$, mostrando o funcionamento do BE SOI nMOSFET

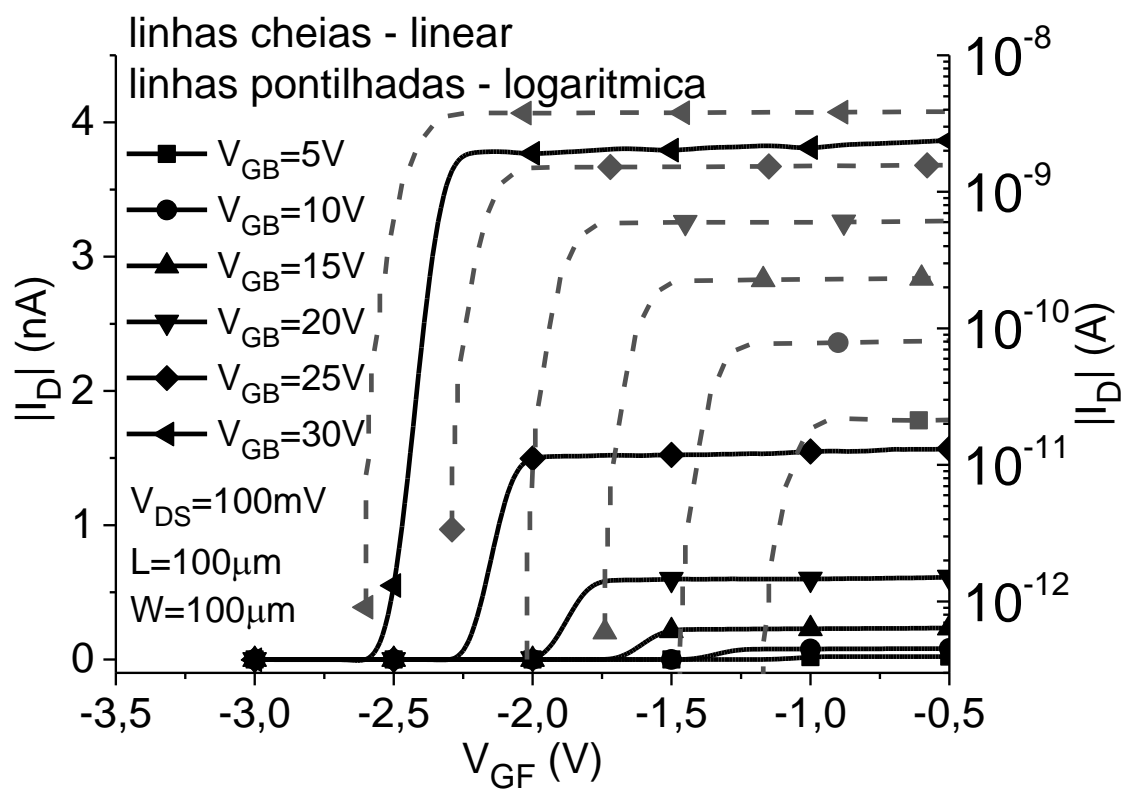

Fonte: Autor.

Para os valores de $\mathrm{V}_{\mathrm{GB}}$ negativos, o transistor se comporta como um BE SOI pMOSFET e para os valores de $\mathrm{V}_{\mathrm{GB}}$ positivos o transistor se comporta como um $\mathrm{BE}$ SOI nMOSFET.

Observamos o aumento da corrente de dreno com o aumento do valor de $\left|\mathrm{V}_{\mathrm{GB}}\right|$ e o deslocamento da tensão de limiar $\mathrm{V}_{\mathrm{T}}$ devido ao aumento de cargas na segunda interface induzidos pelo eletrodo de substrato, assim como ocorria na primeira versão do BE SOI MOSFET.

A corrente de dreno dos transistores BE SOI pMOSFET apresentou-se maior que a corrente dos transistores BE SOI nMOSFET. Este fato ocorre provavelmente devido à um desequilíbrio das barreiras de potencial nos contatos tipo Schottky de fonte e dreno para elétrons e lacunas. A altura da barreira de potencial pode variar dependendo das condições durante a fabricação do dispositivo. Também podem contribuir para essa diferença de corrente o fato de a lâmina utilizada possuir dopagem natural tipo $p$, apesar de ser muito baixa, e possíveis cargas no óxido enterrado.

Foi observado também uma tendência de a corrente de dreno estabilizar (ou variar menos) a partir de um determinado valor de porta $\mathrm{V}_{\mathrm{GF}}$, para ambos tipos de transistores. Para melhor compreender essa característica da segunda versão do BE SOI MOSFET, foram realizadas simulações numéricas deste dispositivo em três 
condições de polarizações distintas. A Figura 5.5 mostra essas três polarizações para - BE SOI pMOSFET (pontos A, B e C). O BE SOI nMOSFET apresenta comportamento análogo.

Figura 5.5 - Curva esquemática da corrente de dreno em função de $V_{G F}$.

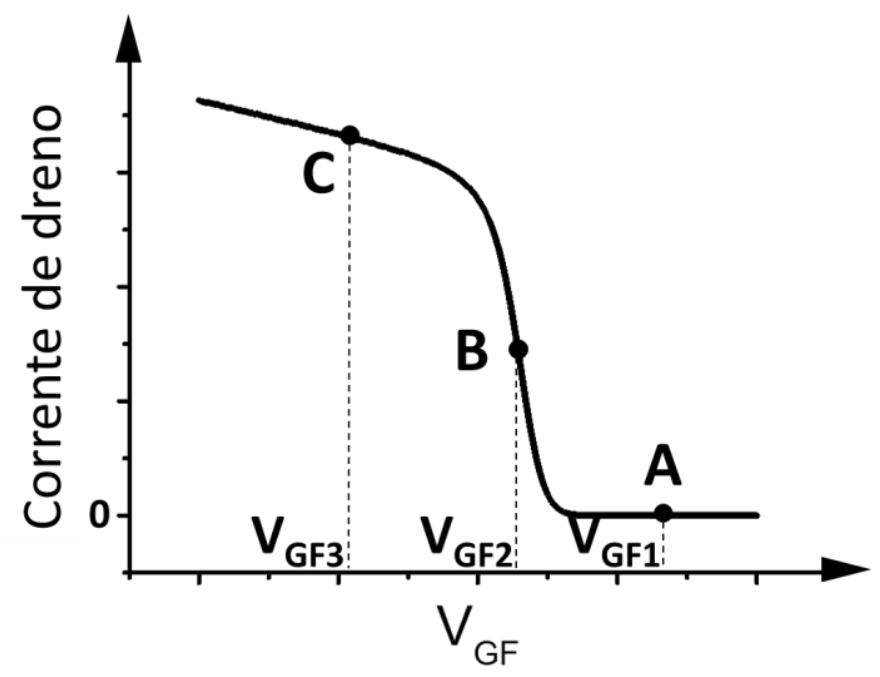

Fonte: Autor.

Figura 5.6 - Simulação numérica da densidade de cargas no corte transversal BB' da Figura 3.2.

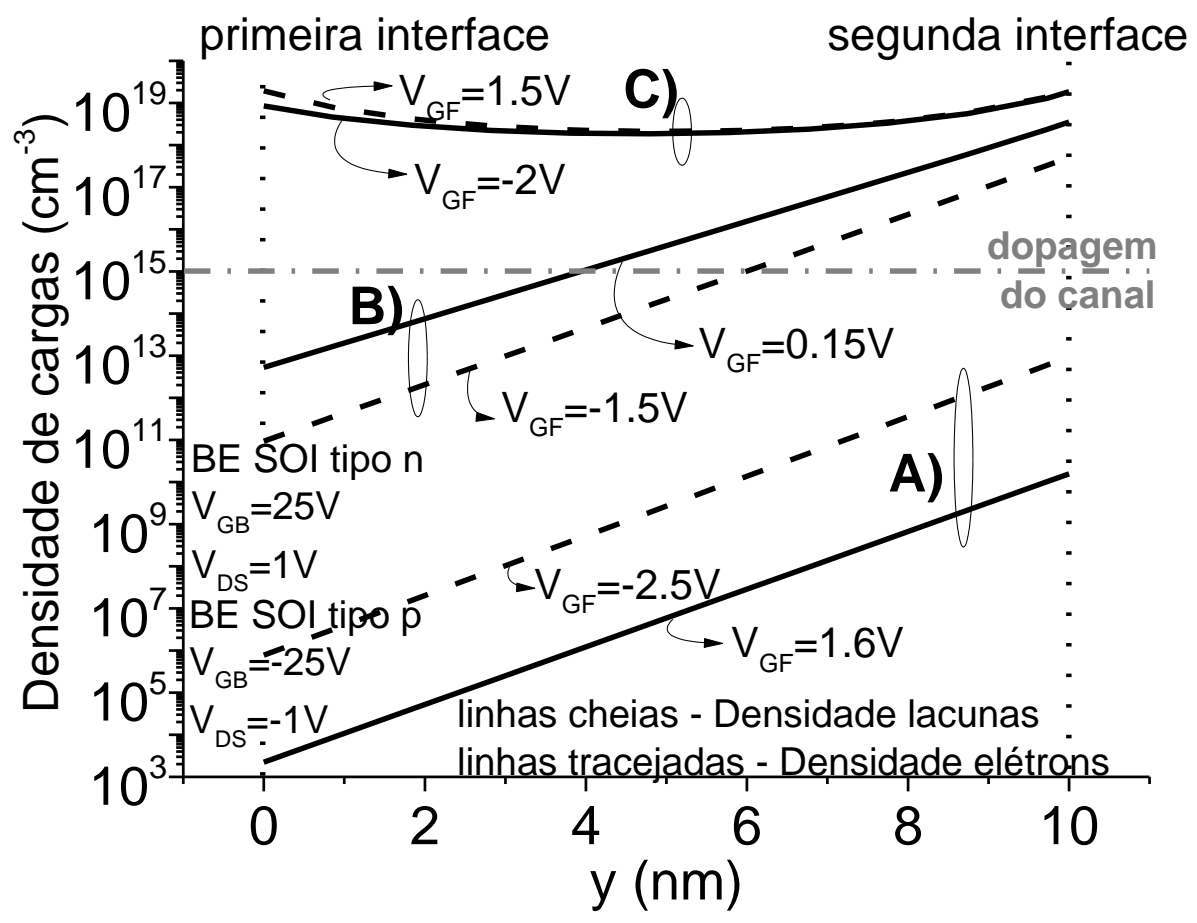

Fonte: Autor. 
A Figura 5.6 mostra o resultado da simulação para densidade de cargas no corte longitudinal BB' da Figura 3.2, correspondendo a segunda interface da camada de silício. As três linhas cheias correspondem a densidade de lacunas, ou seja, correspondem ao transistor BE SOI pMOSFET, enquanto que as linhas tracejadas correspondem a densidade de elétrons, ou seja, o transistor BE SOI nMOSFET. Em cada caso estão representadas três situações de polarização do eletrodo de porta $V_{G F}$ :

- Situação A: transistor em corte (VGF1 na Figura 5.5);

- Situação B: transistor na sua operação convencional (VGF2 na Figura 5.5);

- Situação C: transistor com corrente de dreno estabilizada ( $V_{G F 3}$ na Figura 5.5).

É possível observar a partir da simulação que na situação $A$, a densidade de cargas tanto de lacunas quanto de elétrons é baixa (menor que a dopagem do canal) na primeira e segunda interface. Essa situação corresponde a tensão de porta $\mathrm{V}_{\mathrm{GF}}$ suficiente para depletar completamente a camada de silício abaixo do eletrodo de porta (Figura 5.7A). Não há condução de corrente pois a camada de cargas livres na segunda interface que liga a fonte ao dreno foi interrompida pelo campo elétrico gerado pela porta.

Já na situação $B$, a densidade de cargas aumenta (conforme $V_{G F}$ diminui no caso do BE SOI pMOSFET ou VGF aumenta no caso do BE SOI nMOSFET) em comparação com a situação A. A camada de silício abaixo do eletrodo de porta deixa de estar completamente depletado (Figura 5.7B). Essa densidade de cargas é significativamente maior na segunda interface, indicando que a corrente neste transistor realmente circula nessa região. Nesta condição a curva de transferência do BE SOI MOSFET assume o formato característico, ou seja, a corrente de dreno aumenta conforme $\left|V_{G F}\right|$ aumenta.

A situação C representa uma condição de polarização no qual já é possível observar a corrente de dreno estagnar na curva de transferência do transistor. Nesta condição, a densidade de cargas na segunda interface não aumenta muito mais conforme aumentamos $\left|V_{\mathrm{GF}}\right|$ pois ela é proporcional principalmente à polarização fixa do substrato $V_{G B}$. $A$ polarização da porta $V_{G F}$ influencia majoritariamente a concentração de cargas na primeira interface, que se torna maior do que nas situações anteriores. Porém a corrente circula predominantemente na segunda interface e, 
portanto, a estagnação da densidade de cargas nessa região faz com que a corrente de dreno também se estabilize (Figura 5.7C).

O aumento da densidade de cargas na primeira interface não acarreta no aumento da corrente de dreno. Isto se deve ao fato de a resistência de canal nesta região ser muito elevada devido as distâncias entre o contato de dreno e o canal, e o canal e o contato de fonte (na ordem de alguns micrometros). Essas regiões do silício na primeira interface (fora do eletrodo de porta) não são dopadas e não possuem a camada de cargas livres como há na segunda interface. Portanto a resistência de canal nessa região é muito elevada e o aumento da densidade de cargas pouco influencia a corrente total de dreno. 
Figura 5.7 - Perfis esquemáticos do BE SOI MOSFET em diferentes regiões de operação. A) Operação como transistor em corte. B) Operação como transistor em condução. C) Operação não convencional.
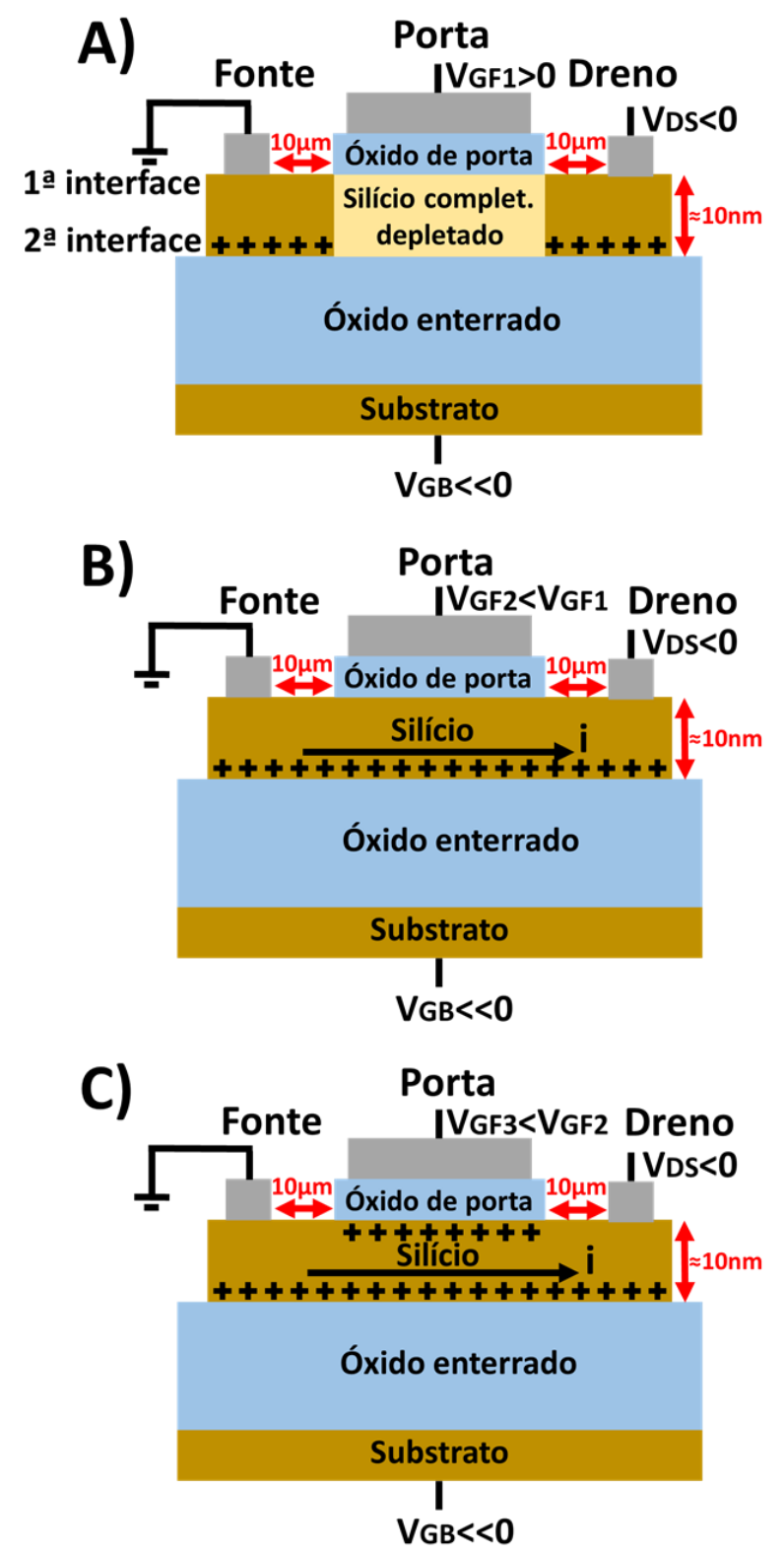

Fonte: Autor.

\subsubsection{Medidas em função da polarização $V_{D S}$}

A Figura 5.8 mostra as medidas elétricas da corrente de dreno em função da tensão $V_{G F}$ realizadas para diversos valores de $V_{D S}$ no transistor BE SOI pMOSFET. Novamente é possível observar que a corrente de dreno tende a estabilizar para um determinado valor de VGF. Nessa situação, o dispositivo se assemelha a um resistor no qual a corrente que passa através dele é proporcional à tensão VDs. 
Também é possível notar uma polarização não convencional [52]. Usualmente a polarização VDS é negativa para transistores pMOSFET, mas nesse caso foi possível utilizar valores positivos de VDs pois como não há junções no BE SOI MOSFET, não há o problema de polarizá-las diretamente.

Figura 5.8 - Curvas de transferência experimentais para diferentes valores de $V_{D S}$ do BE SOI pMOSFET.

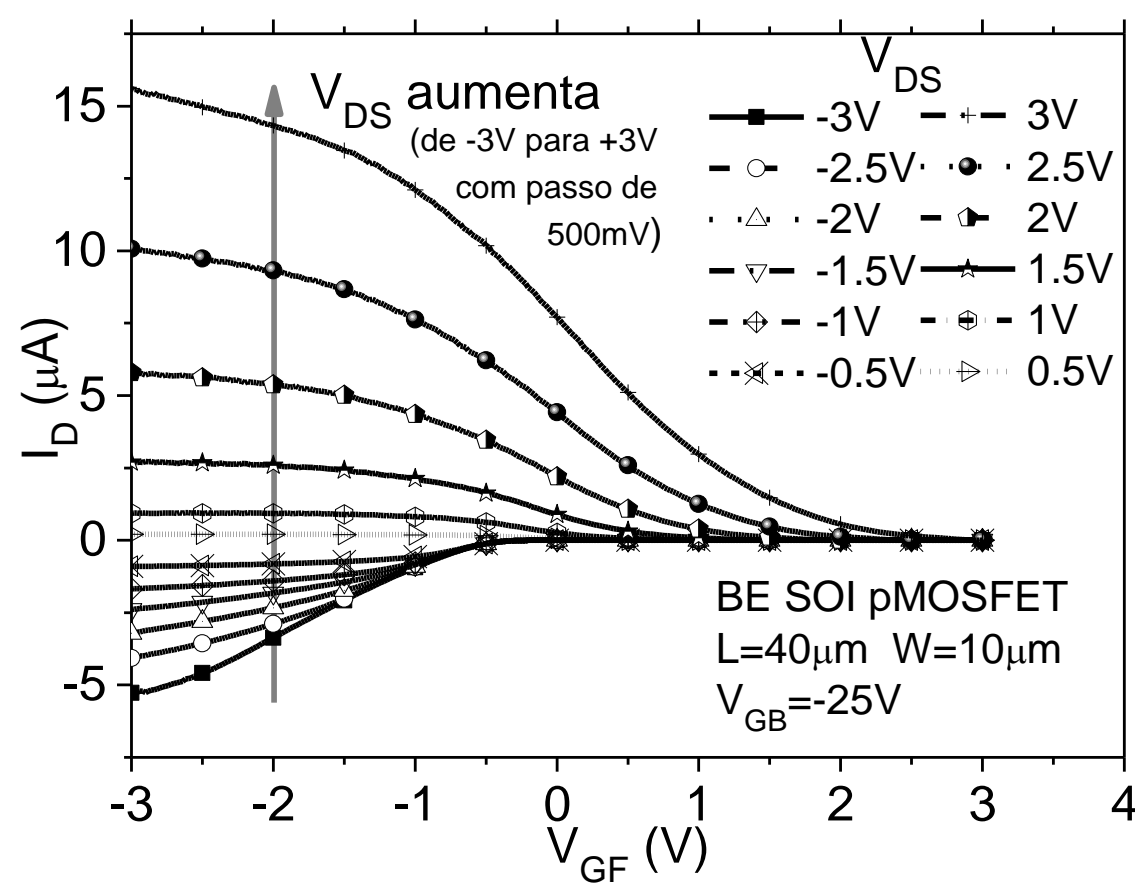

Fonte: Autor.

O nível de corrente para valores de VDs positivos difere do nível de corrente para $V_{D S}$ negativo devido ao leiaute da máscara utilizada para fabricar os dispositivos e devido a diferenças nas resistências de contato da fonte e do dreno. 
Figura 5.9 - Tensão de limiar do BE SOI pMOSFET em função de $V_{D S}$.

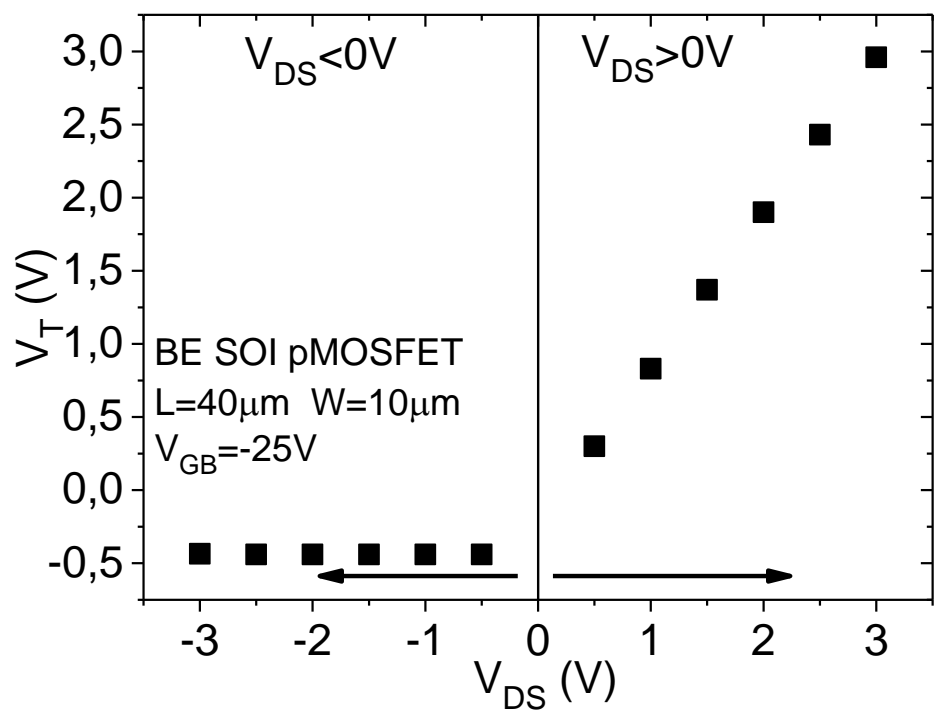

Fonte: Autor.

A Figura 5.9 mostra as tensões de limiar extraídas a partir da Figura 5.8. É possível notar que para valores negativos de $V_{D S}$, a tensão de limiar se mantém praticamente constante. Já para valores positivos, o valor da tensão de limiar aumenta diretamente proporcional à $V_{D S}$.

Figura 5.10 - Curvas de transferência experimentais para diferentes valores de VDS do BE SOI nMOSFET

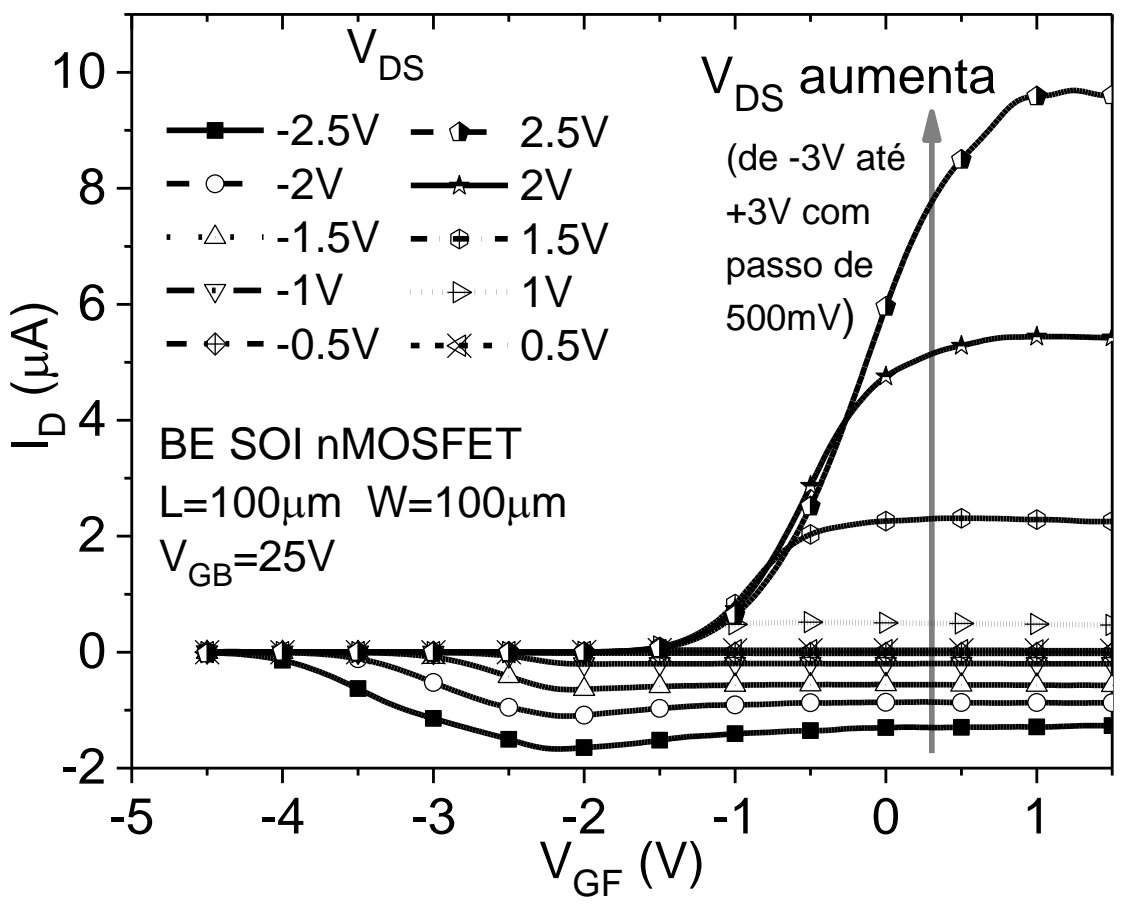

Fonte: Autor. 
As mesmas medidas foram realizadas para o transistor BE SOI nMOSFET, conforme mostrado na Figura 5.10. Um comportamento semelhante ao BE SOI PMOSFET pode ser observado, a corrente apresentou uma tendência a estabilizar a partir de determinado valor de $V_{G F}$. Também é possível notar a polarização não convencional para um transistor tipo $\mathrm{n}$, ou seja, $\mathrm{V}_{\mathrm{DS}}$ negativo, análogo ao $\mathrm{BE} S \mathrm{SO}$ pMOSFET.

Figura 5.11 - Tensão de limiar do BE SOI nMOSFET em função de $V_{D S}$

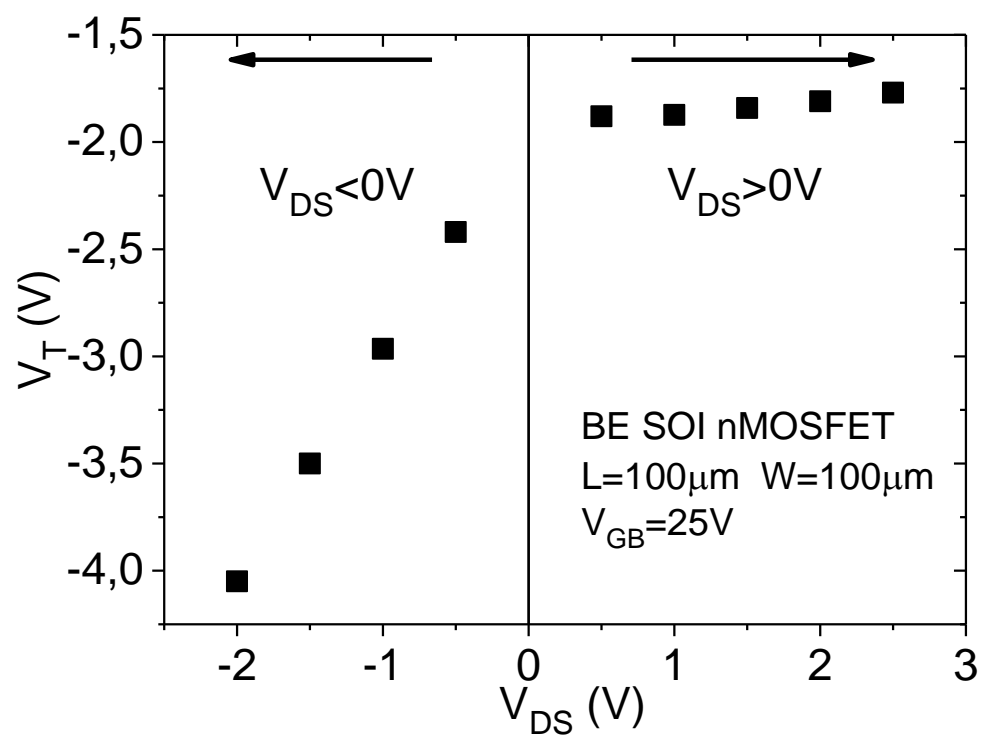

Fonte: Autor.

A tensão de limiar das curvas da Figura 5.10 foram extraídas conforme mostrado na Figura 5.11. O comportamento do BE SOI nMOSFET é análogo ao BE SOI pMOSFET. A tensão de limiar permanece praticamente constante para valores de $V_{D S}$ positivos enquanto que para valores negativos a tensão de limiar é proporcional a $V_{D S}$.

Tanto para os transistores tipo $\mathrm{p}$ quanto para o tipo $\mathrm{n}$, a tensão de limiar na condição de polarização não convencional de $\mathrm{V}_{D S}$ (positivo para o BE SOI pMOSFET e negativo para o BE SOI nMOSFET) variou. A fim de melhor compreender esse comportamento, foram realizadas novas simulações dos dispositivos.

As Figura 5.12 e Figura 5.14 mostram as simulações dos diagramas de bandas de energia para o BE SOI pMSOFET e NMOSFET respectivamente, para valores positivos e negativos de $V_{D S}$. As curvas representam a condição de polarização para um único valor de $V_{G F}$, no qual o transistor ainda está em corte. Essas simulações 
correspondem ao corte $A A^{\prime}$ do perfil esquemático da Figura 3.2, ou seja, na segunda interface da camada de silício.

Figura 5.12 - Simulação numérica do diagrama de bandas de energia do BE SOI pMOSFET na segunda interface para $V_{D S}=-500 \mathrm{mV},-300 \mathrm{mV}$ e $-100 \mathrm{mV}$ (corte $A A^{\prime}$ da Figura 3.2).

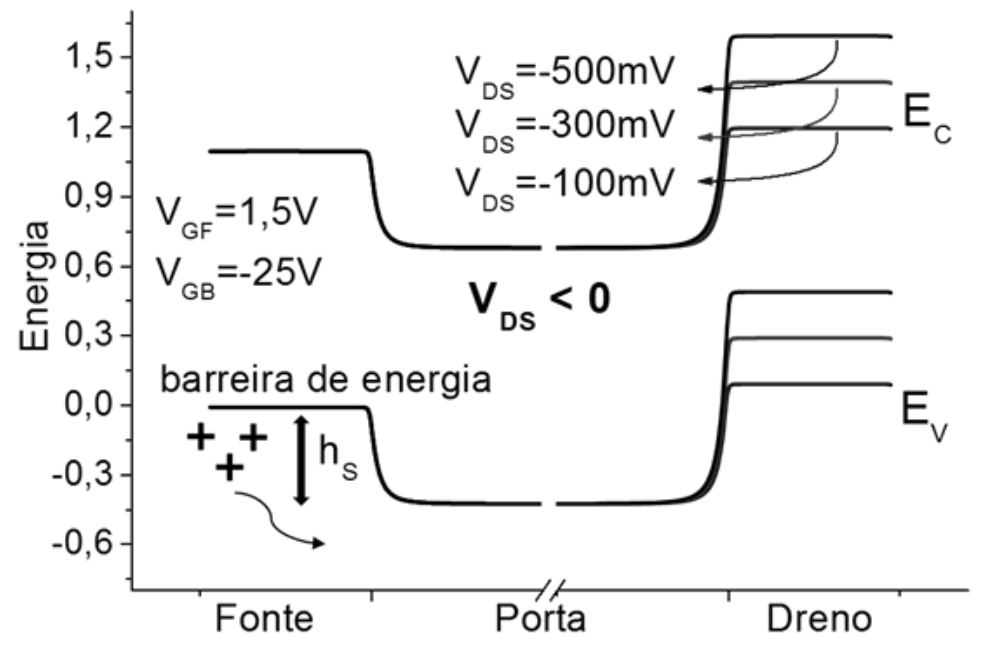

Fonte: Autor.

Figura 5.13 - Simulação numérica do diagrama de bandas de energia do BE SOI pMOSFET na segunda interface para $V_{D S}=100 \mathrm{mV}$ e $200 \mathrm{mV}$ (corte $A A^{\prime}$ da Figura 3.2).

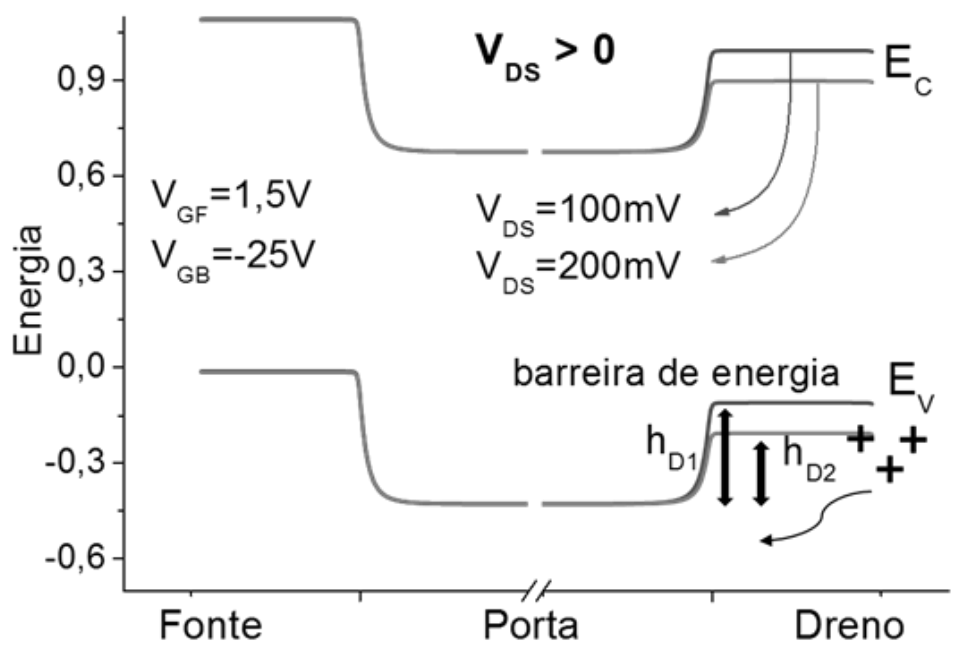

Fonte: Autor.

Para os valores de $V_{D S}$ negativos (-100mV, $-300 m V$ e $\left.-500 m V\right)$, as lacunas que compõe a corrente tendem a fluir no sentido da fonte para o dreno. Logo, a barreira de energia vista pelas lacunas na condição de polarização simulada é aquela entre a fonte e a porta hs (Figura 5.12). A altura hs permanece constante pois o potencial elétrico de referência do eletrodo de fonte é mantido, independentemente do valor de VDS. Como resultado temos que conforme o valor de $\left|V_{G F}\right|$ aumenta, a barreira hs diminui até o ponto em que as lacunas conseguem fluir em direção ao eletrodo de 
dreno (quando $\mathrm{V}_{\mathrm{GF}}=\mathrm{V}_{\mathrm{T}}$ ). Nessa situação há corrente de deriva, o transistor deixa de estar cortado e o valor da tensão de limiar é o mesmo para todos os valores de $V_{D S}$ negativos.

Caso VDS seja positivo (100mV e 200mV na Figura 5.12), as lacunas tendem a fluir no sentido do dreno para a fonte. A barreira de energia vista por elas na condição de corte do transistor depende do valor de VDS. Temos que a altura da barreira para

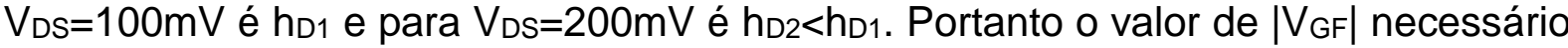
para eliminar a barreira de energia e permitir a passagem de corrente é menor para $V_{D S}=200 \mathrm{mV}$ do que para $V_{D S}=100 \mathrm{mV}$. Logo a tensão de limiar passa a ser diretamente proporcional a VDS caso a tensão entre fonte e dreno seja positiva no BE SOI pMOSFET.

Figura 5.14 - Simulação numérica do diagrama de bandas de energia do BE SOI nMOSFET na segunda interface para $V_{D S}=500 \mathrm{mV}, 300 \mathrm{mV}$ e $100 \mathrm{mV}$ (corte $A A^{\prime}$ da Figura 3.2).

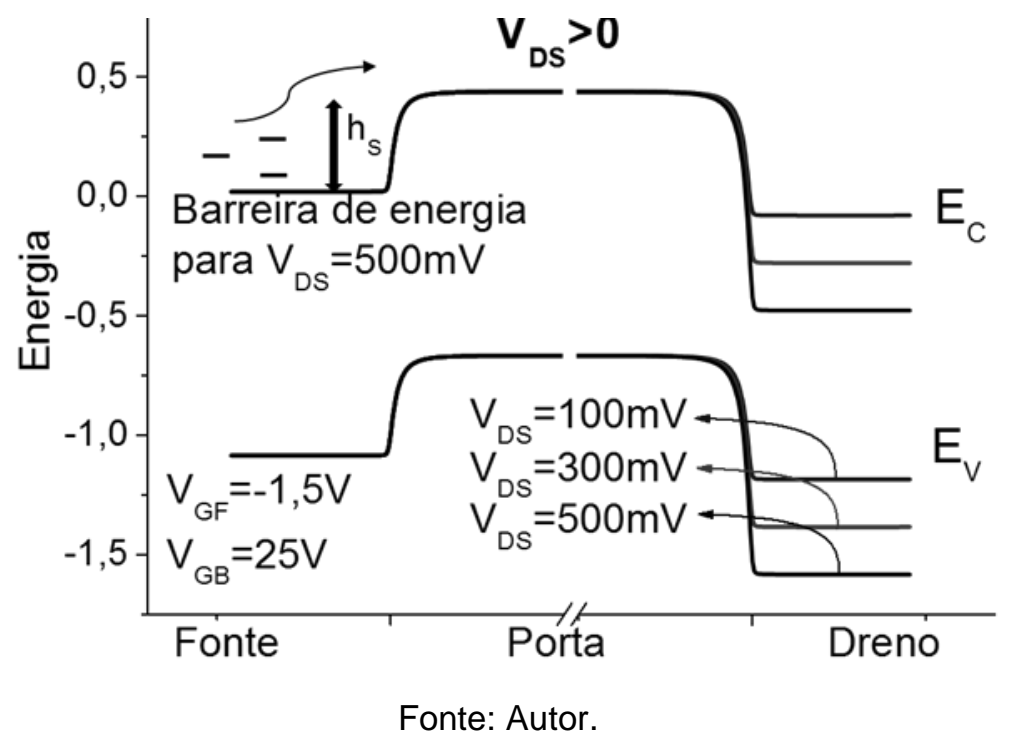


Figura 5.15 - Simulação numérica do diagrama de bandas de energia do BE SOI nMOSFET na segunda interface para $V_{D S}=-100 \mathrm{mV}$ e $-200 \mathrm{mV}$ (corte $A A^{\prime}$ da Figura 3.2).

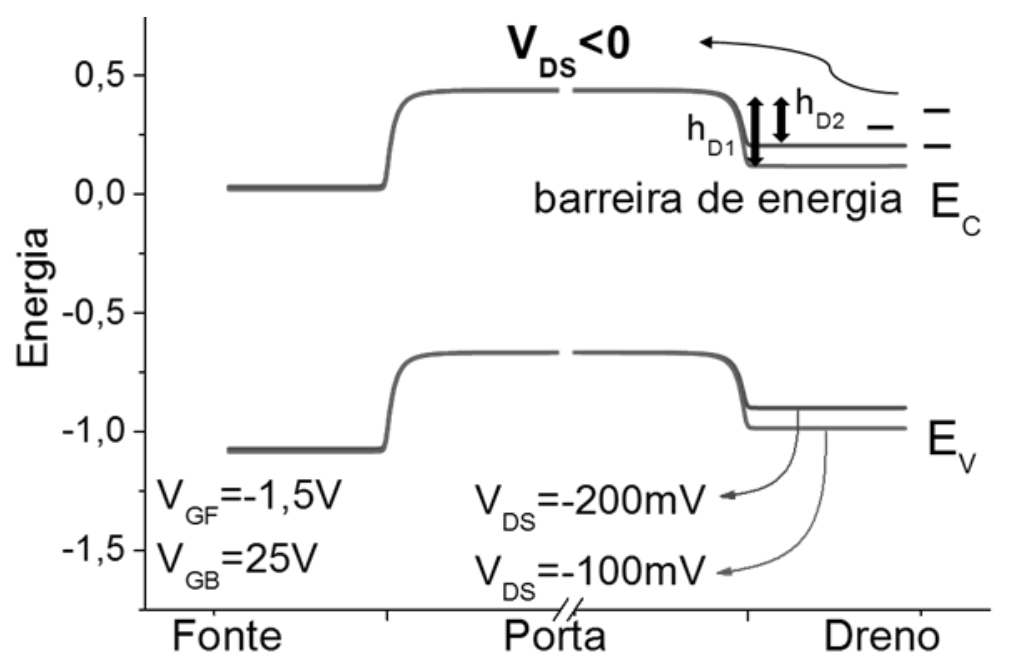

Fonte: Autor.

A tensão de limiar do BE SOI nMOSFET apresenta comportamento análogo devido ao mesmo fenômeno. Quando o valor de $V_{D S}$ for positivo (100mV, 300mV e $500 \mathrm{mV}$ na Figura 5.14), os elétrons que compõe a corrente tendem a fluir do eletrodo de fonte para o dreno. Nessa situação, a barreira de energia vista pelos elétrons quando o transistor está em corte (entre fonte e canal) possui a mesma altura para todos os valores de $V_{D S}>0$ (hs). Como resultado, a tensão de porta $V_{G F}$ necessária para começar a passagem de corrente por deriva é a mesma e, portanto, a tensão de limiar permanece constante.

Caso o valor de $V_{D S}$ seja negativo $(-100 m V$ e $-200 m V$ na Figura 5.14), os elétrons tendem a fluir do dreno para a fonte. A barreira vista pelas cargas entre 0 dreno e o canal tem alturas diferentes dependendo de VDS quando o transistor está em corte. Para esses valores de VDS, temos a altura da barreira de energia hD1 para $V_{D S}=-100 m V$ e a altura da barreira de energia $h_{D 2}<h_{D 1}$ para $V_{D S}=-200 m V$. O valor necessário de $\mathrm{V}_{\mathrm{GF}}$ para eliminar essa barreira de energia é maior para $\mathrm{V}_{\mathrm{DS}}=-100 \mathrm{mV}$ do que para $V_{D S}=-200 \mathrm{mV}$. Logo a tensão de limiar varia proporcionalmente à polarização entre fonte e dreno para $\mathrm{V}_{\mathrm{DS}}<0$ no caso do BE SOI nMOSFET.

\subsubsection{Medidas em altas temperaturas}

Foram realizadas medidas elétricas em função da temperatura para a segunda versão do BE SOI MOSFET. As correntes de dreno em função da tensão $V_{G F}$, para 
temperaturas entre $25^{\circ} \mathrm{C}$ e $150^{\circ} \mathrm{C}$ são mostradas para polarização de substrato $\mathrm{V}_{\mathrm{GB}}=-$ 15V (transistores tipo p, Figura 5.16) e $\mathrm{V}_{\mathrm{GB}}=15 \mathrm{~V}$ (transistores tipo n, Figura 5.17). É possível observar o aumento da corrente para temperaturas mais elevadas em ambos tipos de transistores.

Figura 5.16 - Curvas de corrente $\left|I_{D}\right|$ em função de $V_{G F}$ em função da temperatura para a segunda versão do BE SOI pMOSFET.

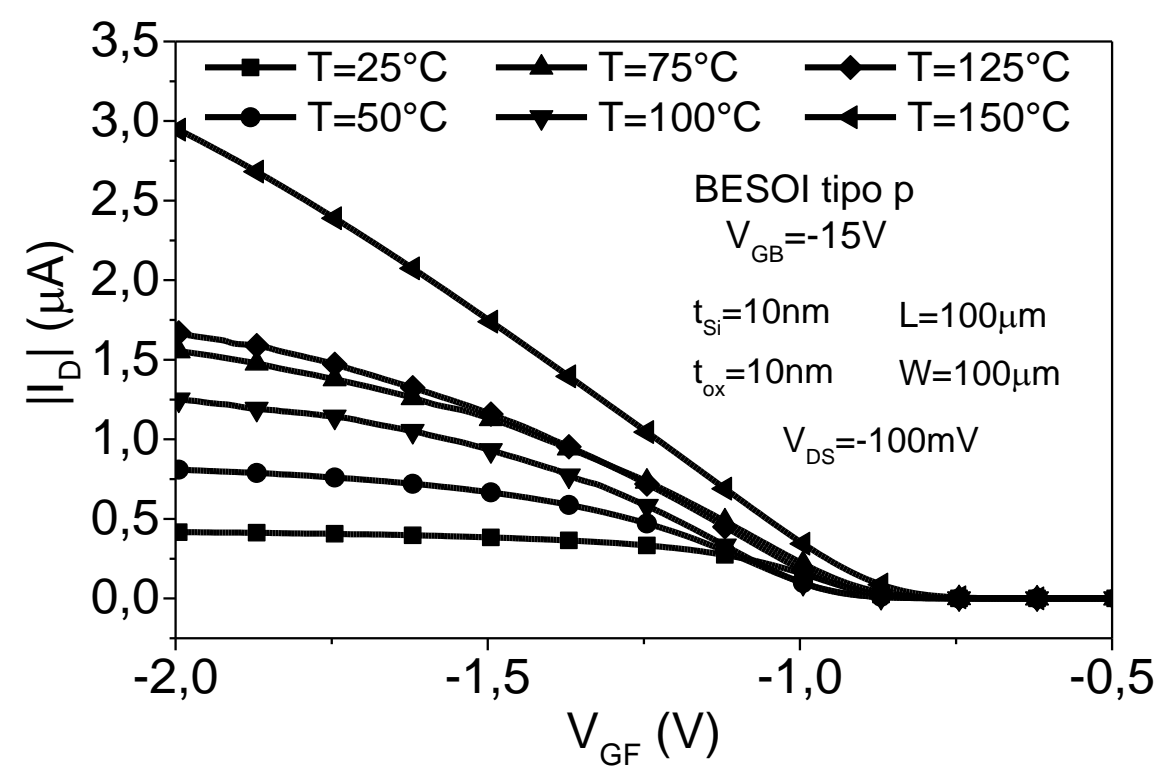

Fonte: Autor.

Figura 5.17 - Curvas de corrente $I_{D}$ em função de $V_{G F}$ em função da temperatura para a segunda versão do BE SOI nMOSFET.

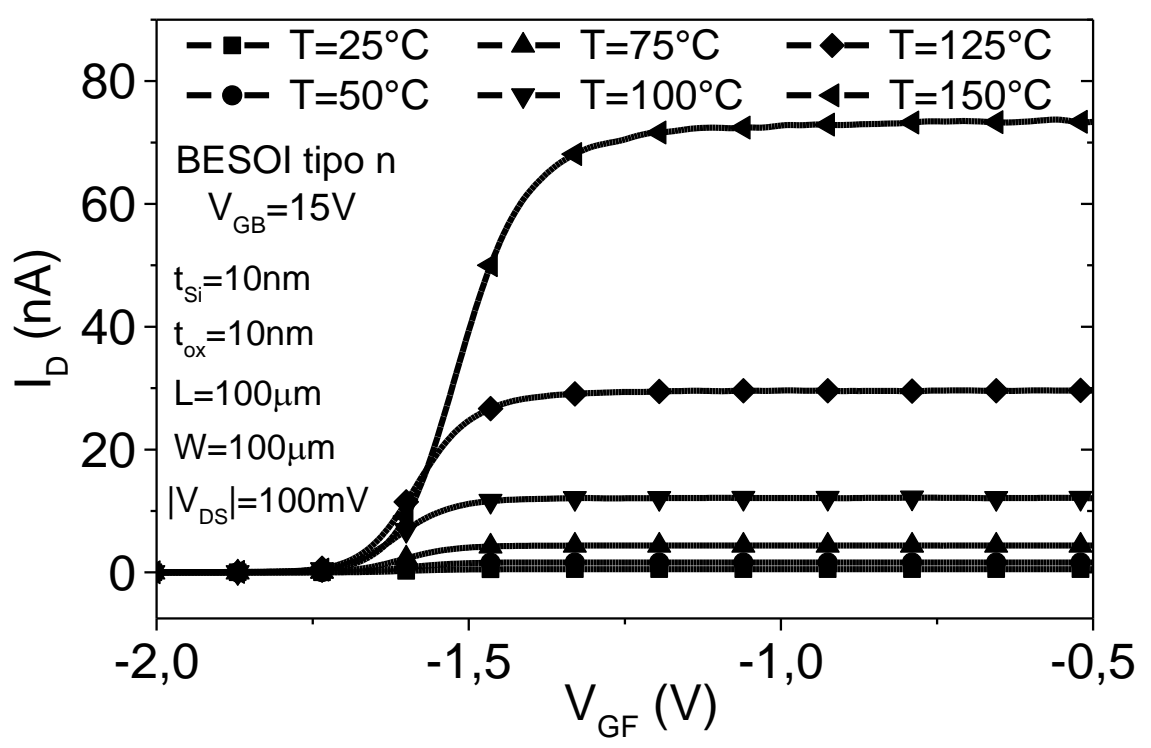

Fonte: Autor. 


\subsubsection{Comparação com a primeira versão do BE SOI}

As medidas apresentadas na Figura 5.16 revelam que, ao contrário da primeira versão do BE SOI MOSFET, a segunda versão não apresentou um ponto de polarização invariante com a temperatura (ZTC). Enquanto que a versão com contatos de alumínio apresentou uma degradação da mobilidade dos portadores conforme elevamos a temperatura, na versão com contatos de níquel o mesmo não foi observado. Como consequência, também não é observado o ponto de ZTC [53].

Simulações para o transistor tipo $\mathrm{p}$ foram realizadas a fim de se investigar os fatores que levam a presença ou não do ZTC. Para evitar a influência das dimensões dos dispositivos, foram comparados os resultados com as mesmas espessuras tsi=23nm e tox=15nm (Figura 5.18, correspondente à primeira versão) em simulações com contatos de fonte e dreno ôhmicos (correspondendo ao alumínio) e com contatos Schottky (correspondendo ao níquel). Em outra simulação, foram comparados os resultados com espessuras tsi=10nm e tox=10nm (Figura 5.19, correspondente à segunda versão) entre contatos ôhmicos e Schottky.

Figura 5.18 - Simulação numérica da corrente de dreno para BE SOI pMOSFET com tsi=23nm e tox $=15 \mathrm{~nm}$ para diferentes temperaturas. A) Contatos ôhmicos. B) Contatos Schottky.

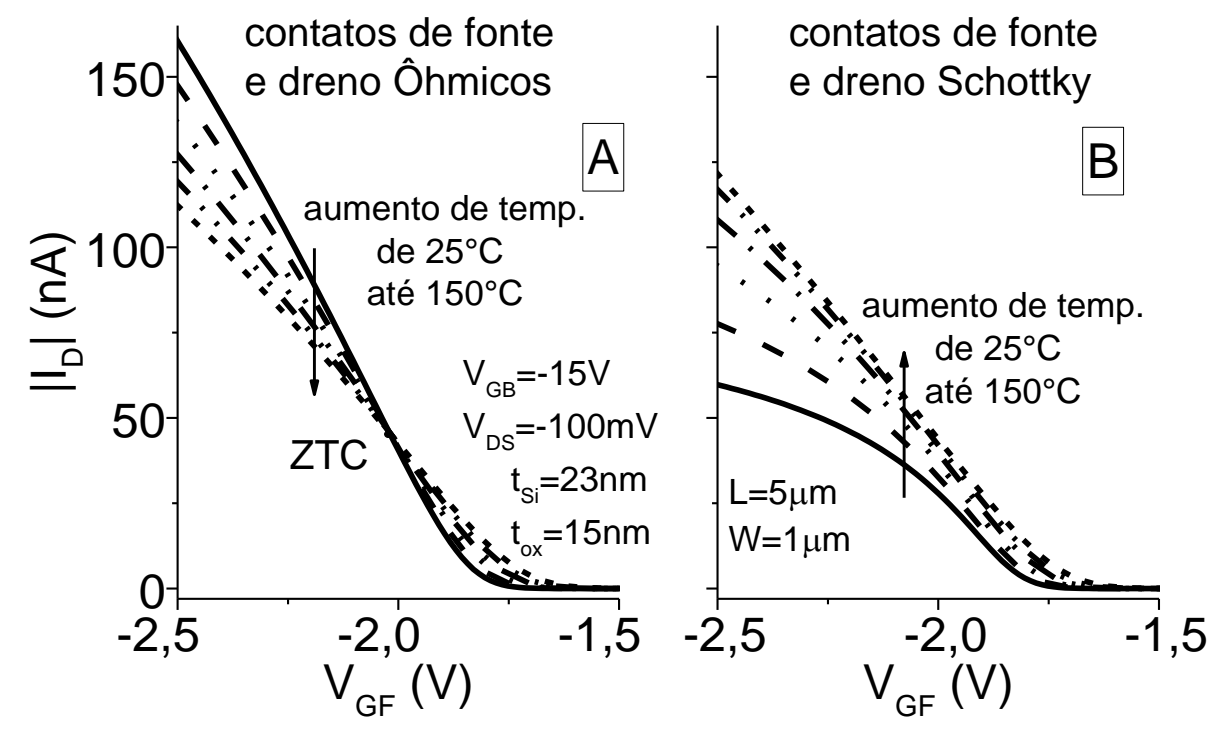

Fonte: Autor. 
Figura 5.19 - Simulação numérica da corrente de dreno para BE SOI pMOSFET com tsi=10nm e tox $=10 \mathrm{~nm}$ para diferentes temperaturas. A) Contatos ôhmicos. B) Contatos Schottky.

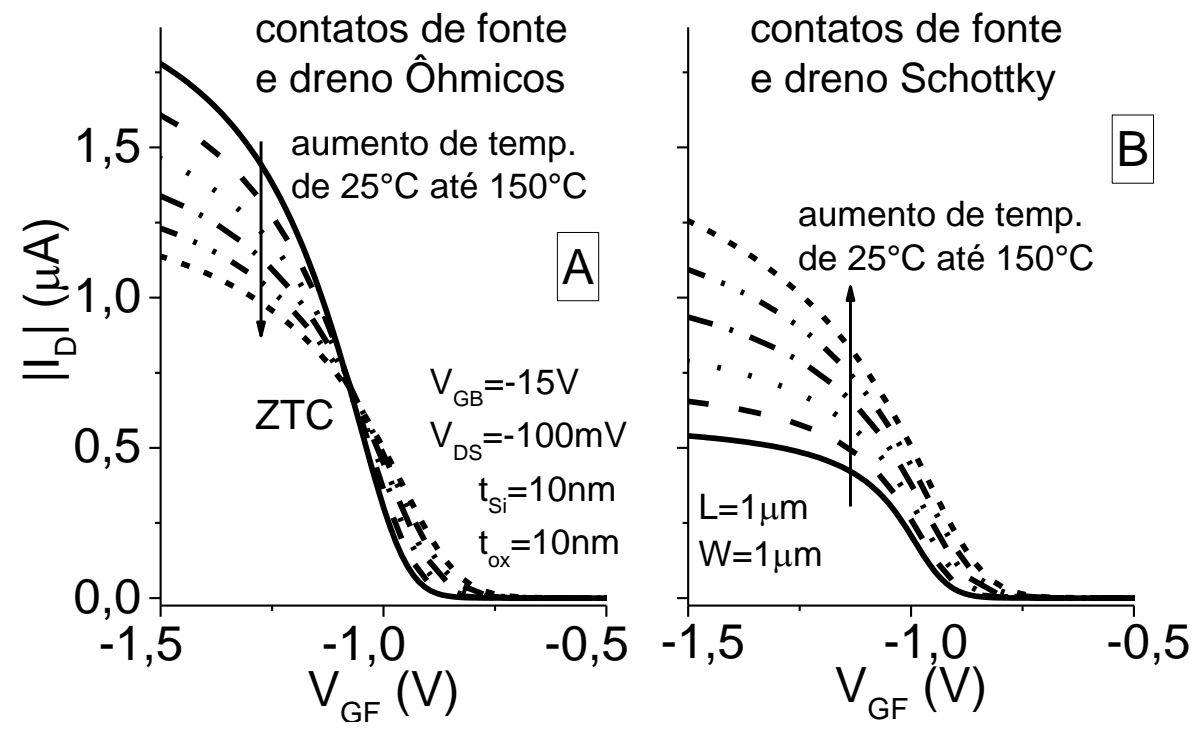

Fonte: Autor.

Em ambas simulações é possível notar que os contatos de fonte e dreno ôhmicos apresentaram uma redução da corrente de dreno em função da temperatura, resultando no ponto de ZTC, conforme resultado obtido experimentalmente. Já para as simulações com contatos Schottky, a corrente de dreno aumentou em função da temperatura, também seguindo os resultados experimentais. Portanto foi possível explorar as simulações para melhor compreender a presença do ZTC em um caso e sua ausência no outro.

Foi analisado o potencial elétrico ao longo do canal na segunda interface (onde a densidade de corrente é mais relevante) nas simulações apresentadas acima. A Figura 5.20 é referente à simulação com tsi $=23 \mathrm{~nm}$ e tox $=15 \mathrm{~nm}$, e a Figura 5.21 à simulação com tsi $=10 \mathrm{~nm}$ e tox $=10 \mathrm{~nm}$. 
Figura 5.20 - Potencial elétrico da simulação numérica do BE SOI pMOSFET com tsi=23nm e $t_{\text {ox }}=15 \mathrm{~nm}$ ao longo da segunda interface para diferentes temperaturas. A) Contatos ôhmicos. B) Contatos Schottky.

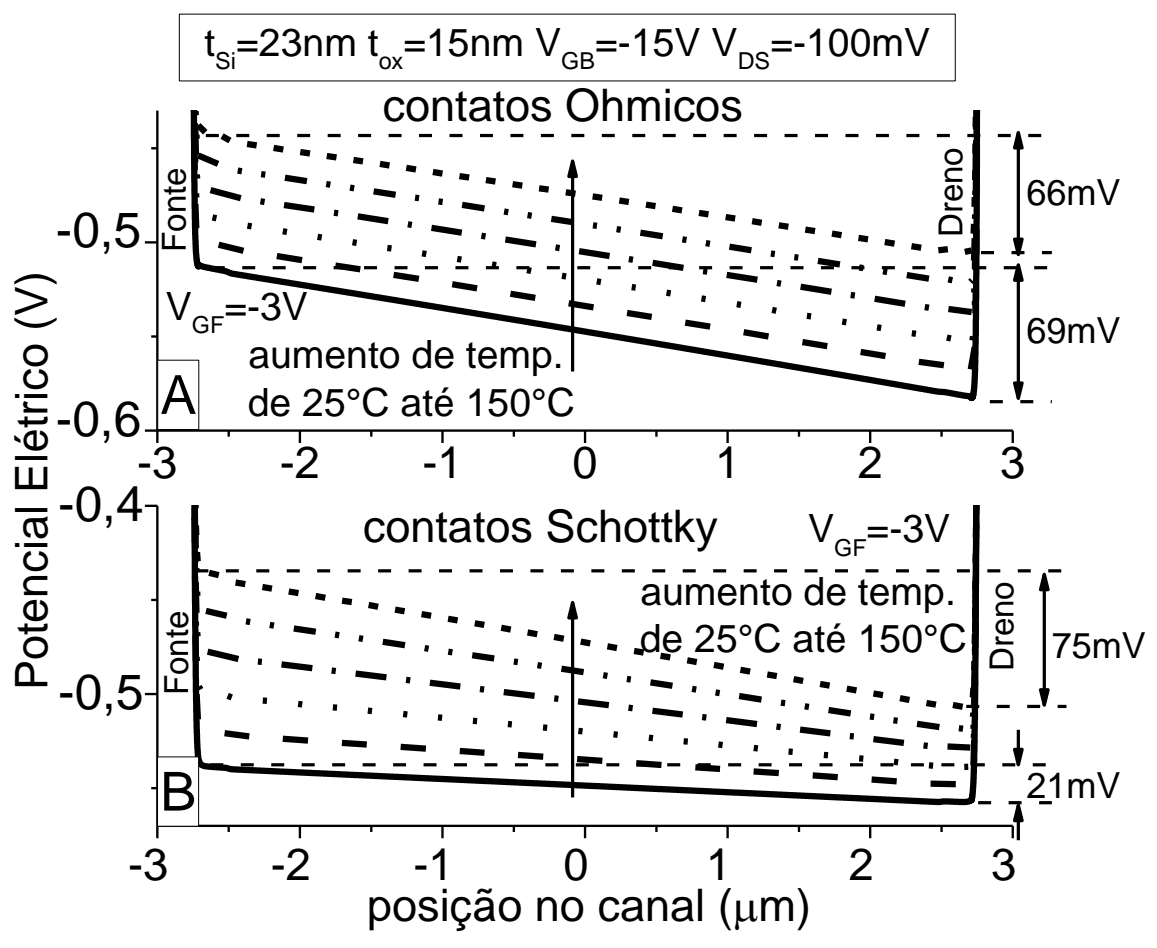

Fonte: Autor.

Figura 5.21 - Potencial elétrico da simulação numérica do BE SOI pMOSFET com tsi=10nm e tox $=10 \mathrm{~nm}$ ao longo da segunda interface para diferentes temperaturas. A) Contatos ôhmicos. B) Contatos Schottky.

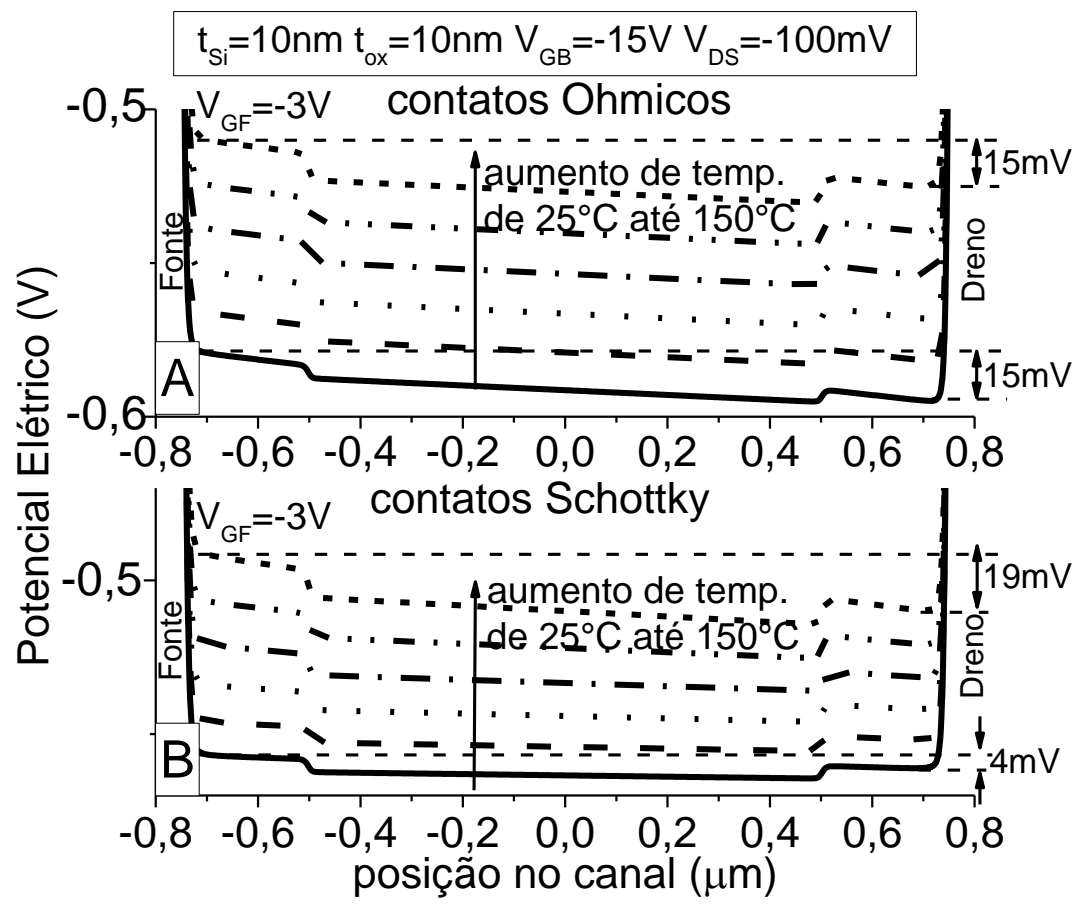

Fonte: Autor. 
Observando ambas figuras é possível notar que a queda de potencial ao longo do canal se mantém constante nas simulações com contatos ôhmicos, independente da temperatura. Com $t_{s i}=23 n m$ e tox $=15 \mathrm{~nm}$ a queda de potencial se manteve entre $66 \mathrm{mV}$ e $69 \mathrm{mV}$ em todas as temperaturas simuladas. Já na outra simulação com tsi=10nm e tox=10nm, esse valor constante foi de $15 \mathrm{mV}$.

No caso das simulações com contatos Schottky o mesmo não ocorre, a queda de potencial aumenta ao longo do canal com a temperatura. Na primeira simulação houve um aumento de $21 \mathrm{mV}$ em $25^{\circ} \mathrm{C}$ para $75 \mathrm{mV}$ em $150^{\circ} \mathrm{C}$, enquanto que na segunda simulação o aumento foi de $4 \mathrm{mV}$ em $25^{\circ} \mathrm{C}$ para $19 \mathrm{mV}$ em $150^{\circ} \mathrm{C}$. Como consequência desse comportamento, observamos o aumento da corrente de dreno com a temperatura. Além disso, esse fenômeno se sobrepõe à redução da mobilidade dos portadores em função da temperatura observado nos dispositivos com contatos ôhmicos.

Os resultados das simulações mostraram que nos dispositivos com contatos Schottky, a queda de potencial ao longo do canal aumenta com a temperatura. Como consequência, a queda de potencial nos contatos de fonte e dreno diminuíram proporcionalmente, uma vez que a mesma polarização $V_{D S}$ foi utilizada em todas as temperaturas simuladas. A redução da queda de potencial nos contatos de fonte e dreno se traduz na diminuição da resistência dos contatos. Portanto a diminuição das resistências de contatos nas junções Schottky com o aumento da temperatura resultou no aumento da corrente de dreno com a temperatura. Já nas simulações com contatos ôhmicos a queda de potencial ao longo do canal se mantém constante e não ocorre o a redução da resistência dos contatos de fonte e dreno com o silício. Neste caso a corrente de dreno acaba reduzindo com a temperatura devido a redução da mobilidade, que passa a ser o fator predominante neste caso.

Podemos observar este fato analisando a densidade de corrente de lacunas no corte transversal do canal (Figura 5.22). Na segunda interface, há um aumento da densidade de corrente de lacunas na simulação com contatos Schottky de 25ำ (linha tracejada preta) para $150^{\circ} \mathrm{C}$ (linha tracejada vermelha). Por outro lado, na simulação com contatos ôhmicos há uma diminuição da densidade de corrente de $25^{\circ} \mathrm{C}$ (linha cheia preta) para $150^{\circ} \mathrm{C}$ (linha cheia vermelha) devido à queda da mobilidade das lacunas com a temperatura. 
Figura 5.22 - Densidade de corrente de lacunas simulada no corte transversal do canal para o transistor BE SOI pMOSFET com $t_{\mathrm{si}}=23 \mathrm{~nm}$.

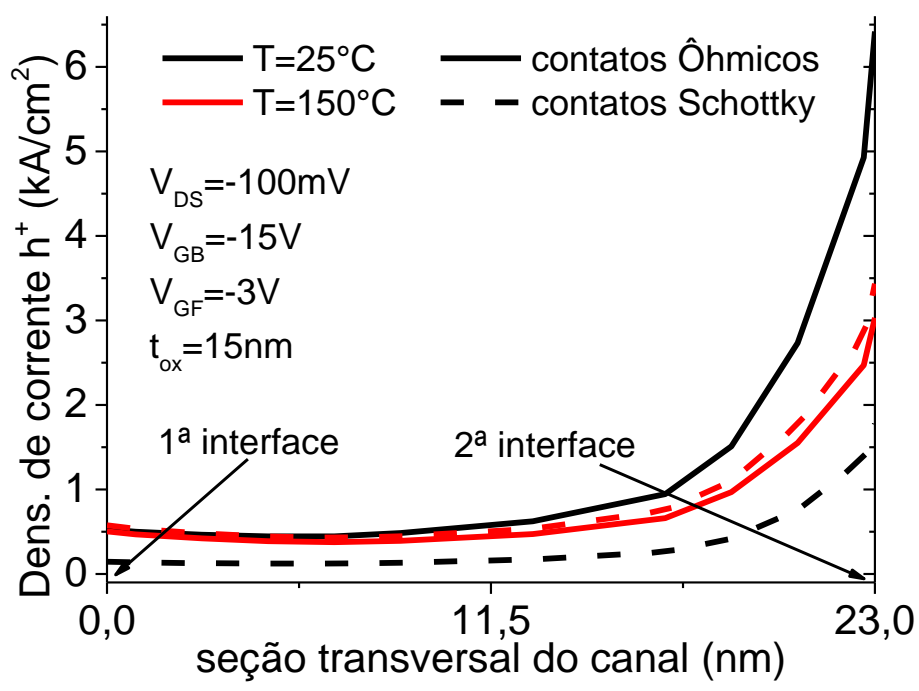

Fonte: Autor.

\subsection{Inversor com BE SOI MOSFET}

Foi construído um circuito inversor utilizando transistores BE SOI MOSFET [54]. Dois transistores fabricados ao mesmo tempo (portanto com características bastante semelhantes) foram polarizados separadamente e conectados externamente, formando o circuito inversor da Figura 2.9. O mesmo circuito poderia ser fabricado em uma única lâmina caso fosse utilizado a tecnologia UTBB SOI, na qual diferentes polarizações poderiam ser aplicadas ao substrato conforme já demonstrado em [55]. Nesse caso também seria necessário uma fonte simétrica para polarização dos substratos do transistor tipo $n$ e tipo $p$.

$\mathrm{O}$ transistor $\mathrm{M} 1$ foi polarizado com $\mathrm{V}_{\mathrm{GB}}=-10 \mathrm{~V}$, formando um BE SOI pMOSFET, enquanto que o transistor $M 2$ foi polarizado com $V_{G B}=10 \mathrm{~V}$, formando um $B E S O I$ nMOSFET. Foi utilizado $V_{D D}=1 \mathrm{~V}$. A curva de transferência de tensão e a corrente do inversor podem ser vistas na Figura 5.23. 
Figura 5.23 - Circuito inversor utilizando o BE SOI MOSFET e a curva de transferência do inversor medida experimentalmente.
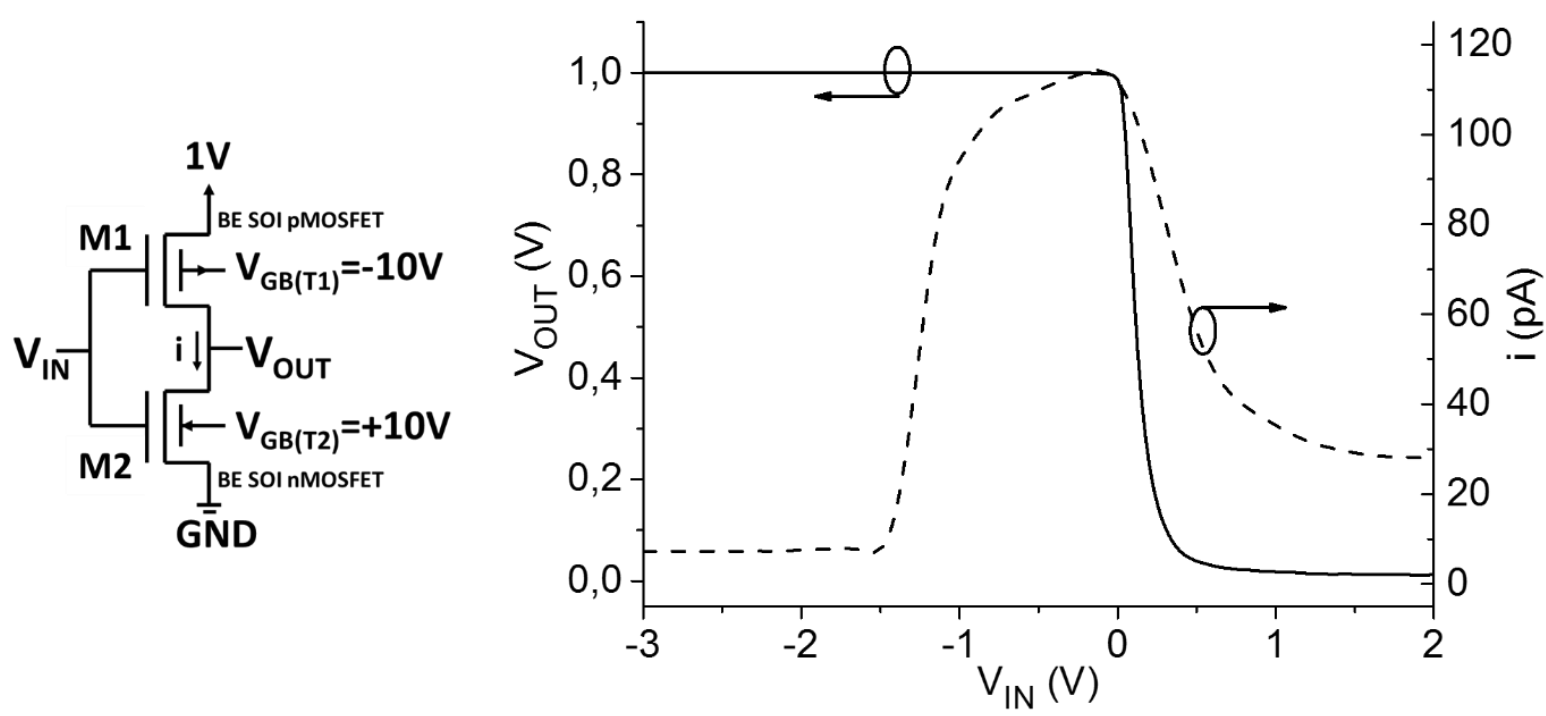

Fonte: Autor.

Nas medidas realizadas podemos destacar duas condições de operação dos transistores. Por volta de $\mathrm{V}_{\mathrm{in}}=2 \mathrm{~V}$, o transistor $\mathrm{M} 2$ está conduzindo enquanto 0 transistor M1 está cortado (apesar de ser possível observar na Figura 5.23 corrente não nula devido a fuga no substrato durante as medidas). Por volta de $\mathrm{V}_{\text {in }}=-2 \mathrm{~V}$ o oposto ocorre, M1 está cortado e M2 conduzindo. Podemos ver também que para esses valores de $\mathrm{V}_{\text {in }}$ a corrente é próxima de zero, apesar de haver uma corrente de fuga para o transistor $M 1$. A transição ocorre por volta de $V_{\text {in }}=0,1 \mathrm{~V}$, nessa região ambos transistores estão conduzindo e a corrente máxima é de aproximadamente $115 p A$.

A fim de se comprovar a flexibilidade dos transistores quanto ao seu tipo (n ou p), foram realizadas medidas elétricas mantendo a mesma configuração do circuito da Figura 5.23, mas invertendo as polarizações. O transistor M1 foi polarizado com

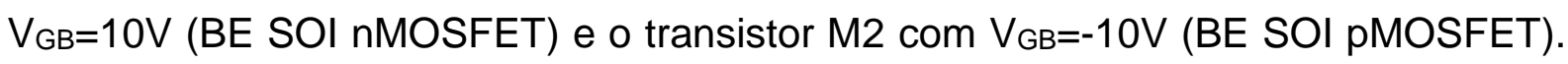
O resultado comparando as duas situações pode ser visto na Figura 5.24, na qual é possível notar uma boa aproximação entre as duas curvas. A diferença máxima observada foi na ordem de $20 \mathrm{mV}$ mostrando que foi possível obter o mesmo circuito inversor alterando a natureza dos transistores. É importante observar que a simplicidade de fabricação do BE SOI MOSFET contribui para a reprodutibilidade de sua operação como transistor reconfigurável. 
Figura 5.24 - Curva de transferência do inversor alternando a polarização do circuito.

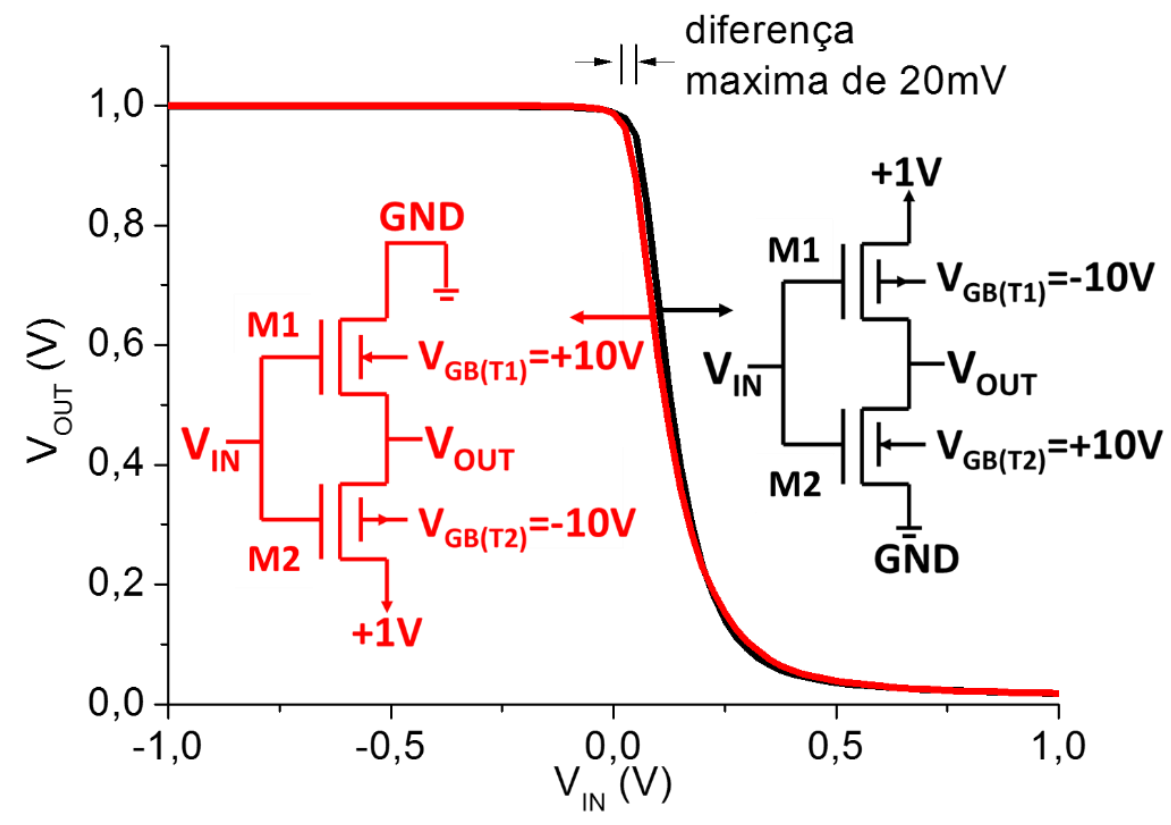

Fonte: Autor.

A Figura 5.24 também nos mostra que, utilizando as condições de polarizações e as dimensões dos transistores mencionadas acima, não foi possível obter uma curva de transferência do inversor simétrica, ou seja, com um ponto de transição igual a $\mathrm{V}_{\mathrm{DD}} / 2$. Inclusive, durante uma faixa de valores de $\mathrm{V}_{\text {in }}$ próximos a transição mais larga que em inversores CMOS convencionais, ambos transistores estão conduzindo, o que contribui para o aumento do consumo de potência.

A fim de tentar promover uma menor dissipação de potência no inversor, foram analisadas as condições em que as tensões de limiar dos transistores BE SOI nMOSFET e BE SOI pMOSFET fossem as mais adequadas possíveis, para que a região em que ambos transistores estão conduzindo fosse a menor possível. Devido ao seu princípio de operação, sabemos que a tensão de limiar é bastante dependente da polarização $V_{\text {GB. }}$ A Figura 5.25 mostra os valores de tensão de limiar extraídos a partir dos dados experimentais. 
Figura 5.25 - Tensão de limiar em função de $V_{G B}$.

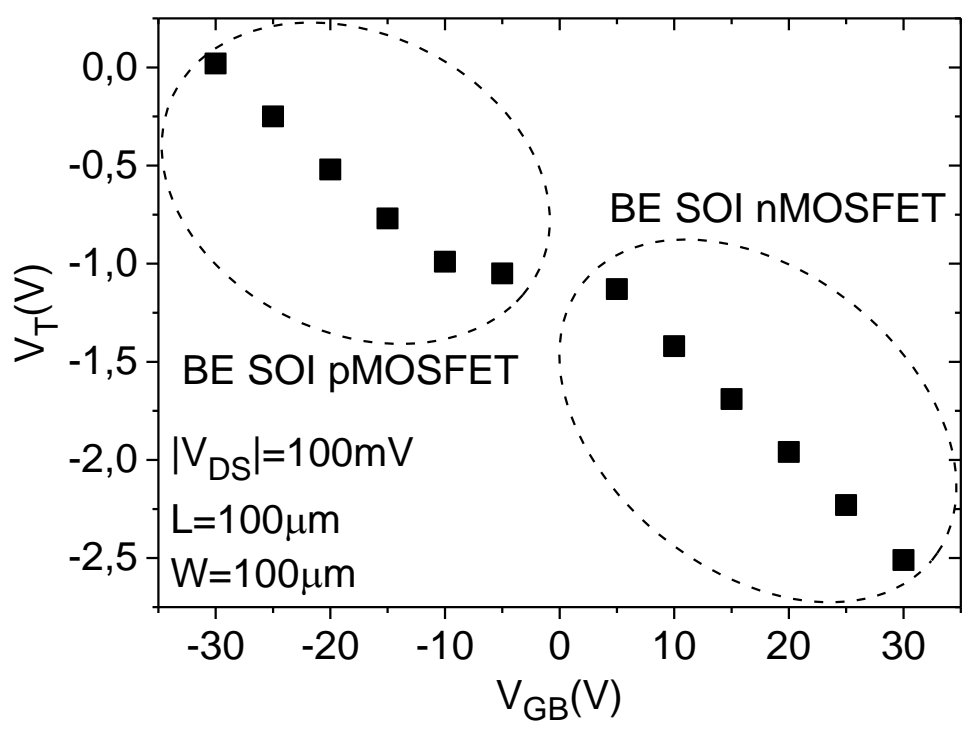

Fonte: Autor.

Para valores baixos de $\left|V_{G B}\right|$ não há formação da camada de lacunas ou elétrons na segunda interface do silício e, portanto, não há formação do transistor (lembrando que $\left|\mathrm{V}_{\mathrm{GB}}\right|$ deve ser elevado o suficiente para a formação do canal devido à espessa camada de óxido enterrado).

Os valores de $\mathrm{V}_{\mathrm{T}}$ dos transistores BE SOI pMOSFETs são sempre maiores que o dos transistores BE SOI nMOSFETs, em um mesmo dispositivo. A Figura 5.25 comprova esse fato. Isso faz com que sempre haja uma faixa de valores de $V_{\text {in }}$ em que ambos transistores estão conduzindo. Portanto a condição em que a corrente do inversor é a menor possível é aquela em que as tensões de limiar dos transistores tipo $\mathrm{p}$ e tipo $\mathrm{n}$ são as mais próximas possíveis, o que diminuiria a faixa de valores de $V_{\text {in }}$ em que ambos estão conduzindo.

Foram realizadas simulações do BE SOI MOSFET alterando as espessuras do óxido de porta e da camada de silício e em seguida foram extraídas as tensões de limiar, com a intenção de se encontrar as melhores condições para reduzir a corrente do inversor. Os resultados das simulações podem ser vistos na Figura 5.26, na qual temos um tox $=10 \mathrm{~nm}$ fixo e tsi variando, e na Figura 5.27, na qual temos um tsi $=10 \mathrm{~nm}$ fixo e tox variando. 
Figura 5.26 - Tensão de limiar simulada do BE SOI MOSFET com tox fixo e tsi variando.

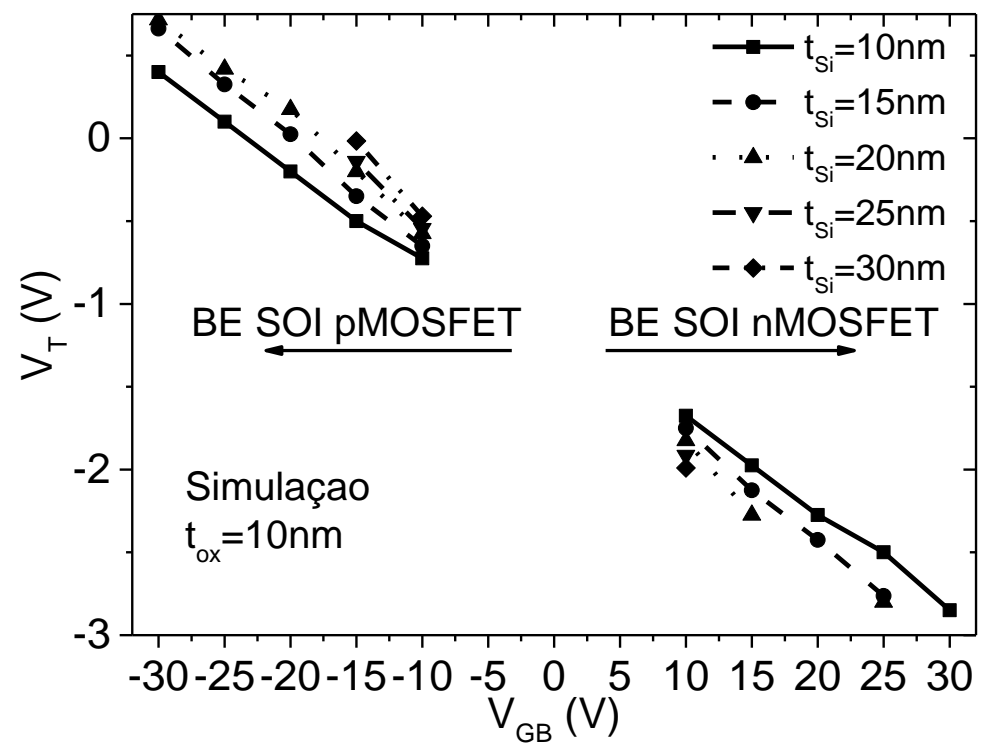

Fonte: Autor.

Figura 5.27 - Tensão de limiar simulada do BE SOI MOSFET com tox variando e tsi fixo.

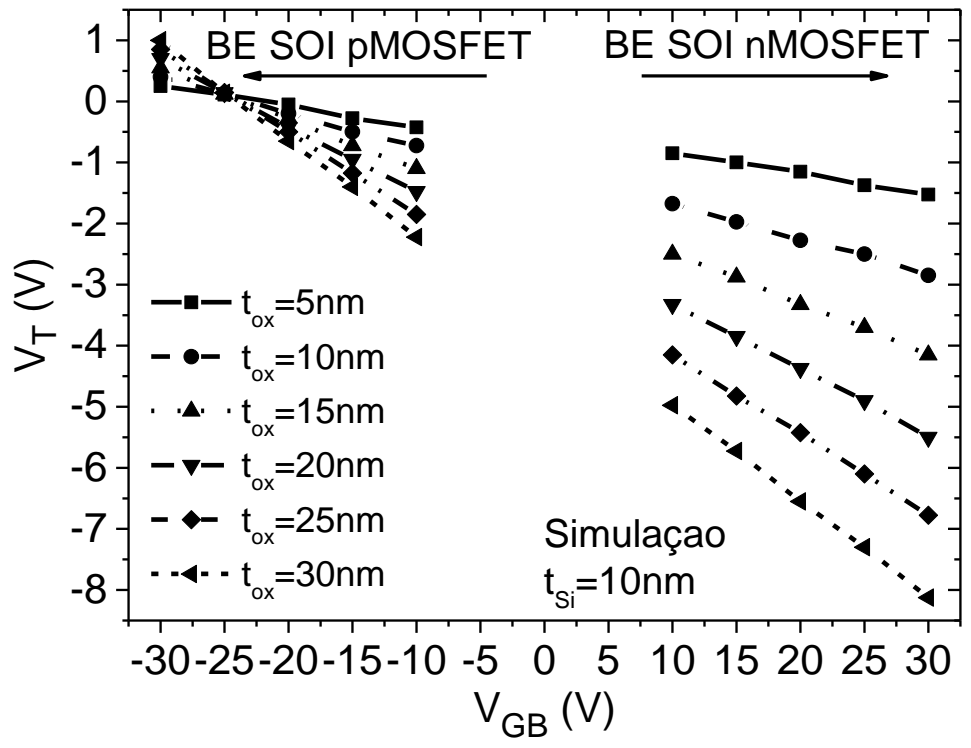

Fonte: Autor.

O fator $\alpha$ dos dispositivos (equivalente a $-\mathrm{dV}_{\mathrm{T}} / \mathrm{dV}_{\mathrm{GB}}$ ) varia de acordo com as espessuras do óxido de porta e da camada de silício, chegando a apresentar até um ponto de cruzamento na Figura 5.27.

A tensão de limiar é mais sensível a variações na espessura do óxido de porta do que variações na espessura do silício. Das Figura 5.26 e Figura 5.27, é possível notar que a variação da tensão de limiar entre os transistores BE SOI pMOSFET e BE 
SOI nMOSFET é menor para as espessuras mais finas de óxido de porta e de silício, e para os menores valores possíveis de $\mathrm{V}_{\mathrm{GB}}$, para os quais ainda há formação de uma camada de cargas na segunda interface. 


\section{Conclusões}

Neste trabalho foi realizado o estudo do transistor BE SOI MOSFET. Como se trata de um novo tipo de transistor, foram feitas medidas elétricas e simulações numéricas para explorar seu princípio de funcionamento e o comportamento dos principais parâmetros elétricos.

Duas versões deste tipo de transistor foram analisadas.

$\mathrm{Na}$ primeira versão (com contatos de fonte e dreno ôhmicos utilizando o alumínio) foram realizadas medidas em altas temperaturas, tendo em vista a importância de diversas aplicações que exigem circuitos operando nessa condição. Devido ao seu princípio de operação, foi visto uma grande dependência da tensão de limiar com a polarização de substrato $\mathrm{V}_{\mathrm{GB}}$.

Os resultados experimentais obtidos a partir do BE SOI pMOSFET mostraram um aumento da tensão de limiar em função da temperatura de até $1,5 \mathrm{mV} /{ }^{\circ} \mathrm{C}$. Também foi observado um fator de degradação da mobilidade com a temperatura c de até 1,3. A inclinação de sublimiar apresentou uma forte degradação com a temperatura. Além disso, foi constatado a presença do ponto invariante com a temperatura (ZTC) neste dispositivo como resultado de dois fenômenos: a variação da tensão de limiar e a degradação da mobilidade com o aumento de temperatura. Apesar de não ser desenvolvido para o BE SOI MOSFET, foi verificado que o modelo Camillo-Martino para o cálculo de $V_{\text {ZTC }}$ apresentou uma boa concordância com os resultados experimentais.

Nesta primeira versão, não foram obtidas medidas satisfatórias para o transistor BE SOI nMOSFET. O principal motivo disto é o metal utilizado nos eletrodos de fonte e dreno, no caso alumínio, que devido sua natureza ôhmica não favorecem a formação da camada de elétrons na segunda interface.

Ao contrário da primeira versão, na segunda versão (com contatos de fonte e dreno de níquel) foi possível observar o BE SOI MOSFET operando como transistor tipo $n$. A sua reconfigurabilidade foi atingida mudando o metal dos eletrodos de fonte e dreno, agora níquel. Devido a sua função trabalho, barreiras de potencial mais simétricas foram criadas nos contatos de fonte e dreno, permitindo que fossem criados 
tanto uma camada de elétrons quanto uma camada de lacunas na segunda interface, dependendo do valor de $\mathrm{V}_{\mathrm{GB}}$.

Foi observado que, ao contrário de transistores MOSFET convencionais, a polarização entre fonte e dreno para o BE SOI MOSFET pode ser positiva ou negativa em uma larga faixa de valores para transistores tipo $n$ e tipo $\mathrm{p}$.

Medidas em função da temperatura mostraram que a segunda versão do BE SOI MOSFET não apresentou o ponto de ZTC como na primeira versão. Simulações numéricas comparando apenas a influência do tipo de contato de fonte e dreno mostraram que com contatos Schottky há uma redução da resistência de contato no dispositivo conforme aumentamos a temperatura. Com isso observamos uma maior queda de potencial elétrico ao longo do canal e uma maior corrente de dreno em função da temperatura. Como resultado, não há a presença do ponto de ZTC pois o aumento do potencial entre fonte e dreno (decorrente da diminuição da resistência de contato) se sobrepõe a degradação da mobilidade com a temperatura.

A construção de um circuito inversor utilizando transistores BE SOI MOSFET mostrou ser viável para esse tipo de aplicação. Ao inverter as polarizações dos transistores de modo que as naturezas dos transistores fossem trocadas, a mesma funcionalidade do circuito inversor foi obtida. No inversor realizado, há passagem de corrente elétrica em uma larga faixa de valores de $V_{\text {in }}$, que pode ser reduzida ao se fabricar transistores BE SOI MOSFET com menores espessuras de óxido de porta e de silício.

Dentre as principais características do BE SOI MOSFET, podemos destacar a sua simplicidade de fabricação, exigindo apenas processos bem conhecidos e nenhuma etapa de dopagem do silício. Outra característica relevante é sua reconfigurabilidade, podendo atuar como transistor tipo $\mathrm{p}$ ou tipo $\mathrm{n}$ dependendo apenas da polarização do substrato.

Como sequência do trabalho sugere-se a fabricação do BE SOI MOSFET utilizando lâminas UTBB, ou seja, com a camada de silício e óxido enterrado ultrafinas (cerca de uma dezena de nanômetros). Com essa estrutura seria possível diminuir os valores de $V_{G B}$ necessários para a formação da camada de elétrons e de lacunas na segunda interface, quando comparado aos valores utilizados neste trabalho. Outra possibilidade inspirada neste trabalho seria explorar o metal de contato de fonte e 
dreno que tornasse os valores de corrente de dreno do BE SOI MOSFET tipo p e tipo n simétricos, já que foi observado a dependência deste material nos valores de corrente. 


\section{PUBLICAÇÕES GERADAS DURANTE O MESTRADO}

Padovese, J. A.; Yojo, L. S.; Rangel, R. C.; Martino, J. A.; "Back Enhanced SOI pMOSFET with Dynamic Threshold Voltage Configuration" em SEMINATEC, 2016.

Yojo, L. S.; Padovese, J. A.; Rangel, R. C.; Martino, J. A.; "Back Enhanced (BE) SOI pMOSFET Behavior at High Temperatures" em SBMicro, 2016.

Yojo, L. S.; Rangel, R. C.; Sasaki, K. R. A.; Martino, J. A.; "BE SOI MOSFET: A Very Simple Reconfigurable SOI Transistor" em SEMINATEC, 2017.

Yojo, L. S.; Rangel, R. C.; Sasaki, K. R. A.; Martino, J. A.; "Back Enhanced (BE) SOI MOSFET under non-conventional bias conditions" em EUROSOI-ULIS, 2017.

Yojo, L. S.; Rangel, R. C.; Sasaki, K. R. A.; Martino, J. A.; "Reconfigurable Back Enhanced (BE) SOI MOSFET used to Build a Logic Inverter" em SBMicro, 2017.

Yojo, L. S.; Rangel, R. C.; Sasaki, K. R. A.; Martino, J. A.; "Is there a Zero Temperature Bias Point (ZTC) on Back Enhanced (BE) SOI MOSFET?" em S3S Conference, 2017. 


\section{Referências}

[1] J. S. Kilby, "Invention of the integrated circuit," IEEE Transactions on Electron Devices, 1976.

[2] D. Kahng e J. Atalla, "Silicon-silicon dioxide field induced surface devices," IREAIEE Solid State Device Research Conference, 1960.

[3] W. F. Brinkman, D. E. Haggan e W. W. Troutman, "A history of the invention of the transistor and where it will lead us," IEEE Journal of Solid-State Circuits, pp. $1858-1865,1997$.

[4] K. Rupp, "40 Years of Microprocessor Trend Data," [Online]. Available: https://github.com/karlrupp/microprocessor-trend-data. [Acesso em 1902 2018].

[5] G. E. Moore, "Cramming more components onto integrated circuits," Electronics, 1965.

[6] H. W. Lam, "Laser-recrystallized silicon-on-oxide - The ideal silicon-on-insulator structure for VLSI?," International Electron Devices Meeting, pp. 556 - 558, 1980.

[7] R. R. Troutman, Latchup in CMOS Technology: The Problem and Its Cure, Kluwer Academic Publishers, 1986.

[8] J. P. Colinge, "Thin-film SOI devices: A perspective," Microelectronic Engineering, pp. 127-147, 1988.

[9] J. P. Colinge, "Thin-film SOI technology: the solution to many submicron CMOS problems," Technical Digest of EDM, pp. 817-820, 1989.

[10] J. P. Colinge, Silicon-On-Insulator Technology: Materials to VLSI, $3^{\text {a }}$ ed., Kluwer Academic Publishers, 2004.

[11] W. A. Krull e J. C. Lee, "Demonstration of the benefits of SOI for high temperature operation," Proceedings of SOS/SOI Technology Workshop, 1988. 
[12] D. Flandre, A. N. Nazarov e P. L. F. Hemment, Science and Technology of Semiconductor-On-Insulator Structures and Devices Operating in a Harsh Environment, Springer, 2005.

[13] R. W. Russell e T. M. Frederiksen, "Automotive and industrial electronic building blocks," IEEE Journal of Solid-State Circuits, pp. 446 - 454, 1972.

[14] F. S. Shoucair, "Potential and problems of high-temperature electronics and CMOS integrated circuits $\left(25-250^{\circ} \mathrm{C}\right)$ - an overview," Microelectronics Journal, pp. 39-54, 1991.

[15] F. S. Shoucair, "Analytical and experimental methods for zero-temperaturecoefficient biasing of MOS transistors," Electronics Letters, pp. 1196 - 1198, 1989.

[16] F. M. Klaassen e W. Hes, "On the temperature coefficient of the MOSFET threshold voltage," Solid-State Electronics, pp. 787-789, 1986.

[17] L. Le Pailleur, "Fully-depleted-silicon-on-insulator from R\&D concept to industrial reality," S3S, 2013.

[18] T. Chiarella, L. Witters, A. Mercha, C. Kerner, M. Rakowski, C. Ortolland, L. A. Ragnarsson, B. Parvais, A. De Keersgieter, S. Kubicek, A. Redolfi, C. Vrancken, S. Brus, A. Lauwers, P. Absil, S. Biesemans e T. Hoffmann, "Benchmarking SOI and bulk FinFET alternatives for PLANAR CMOS scaling succession," Solid-State Electronics, pp. 855-860, 2010.

[19] L. Gaben, S. Barraud, M.-P. Samson, M.-A. Jaud, S. Martinie, O. Rozeau, J. Lacord, C. Arvet, C. Vizioz, J. Bustos, J.-A. Dallery, S. Pauliac, V. Balan, C. Euvrard-Colnat, C. Perrot, V. Loup, P. Besson, J.-M. Hartmann, S. Monfray, F. Boeuf, T. Skotnicki, F. Balestra e M. Vinet, "Evaluation of Stacked Nanowires Transistors for CMOS: Performance and Technology Opportunities," ECS Transactions, pp. 43-54, 2016.

[20] C. Navarro, S. Barraud, S. Martinie, J. Lacord, M. A. Jaud e M. Vinet, "Reconfigurable field effect transistor for advanced CMOS: A comparison with FDSOI devices," EUROSOI-ULIS, 2016. 
[21] T. Baldauf, A. Heinzig, T. Mikolajick, W. M. Weber e J. Trommer, "Strainengineering for improved tunneling in reconfigurable silicon nanowire transistors," EUROSOI-ULIS, 2016.

[22] F. Wessely, T. Krauss e U. Schwalke, "Reconfigurable CMOS with undoped silicon nanowire midgap Schottky-barrier FETs," Microelectronics Journal, pp. 1072-1076, 2013.

[23] A. Heinzig, S. Slesazeck, F. Kreupl, T. Mikolajick e W. A. Weber, "Reconfigurable Silicon Nanowire Transistors," Nano Letters, p. 119-124, 2012.

[24] T. Krauss, F. Wessely e U. Schwalke, "Electrically reconfigurable dual metal-gate planar field-effect transistor for dopant-free CMOS," International MultiConference on Systems, Signals \& Devices (SSD), 2016.

[25] J. Trommer, A. Heinzig, S. Slesazeck, T. Mikolajick e W. M. Weber, "Elementary Aspects for Circuit Implementation of Reconfigurable Nanowire Transistors," IEEE Electron Device Letters, pp. 141 - 143, 2014.

[26] M. Simon, A. Heinzig, J. Trommer, T. Baldauf, T. Mikolajick e W. M. Weber, "Bringing reconfigurable nanowire FETs to a logic circuits compatible process platform," Nanotechnology Materials and Devices Conference (NMDC), 2016.

[27] U. Schwalke, T. Krauss e F. Wessely, "Dopant-Free CMOS on SOI: Multi-Gate Si-Nanowire Transistors for Logic and Memory Applications," ECS Transactions, pp. 105-114, 2013.

[28] J. Trommer, A. Heinzig, T. Baldauf, S. Slesazeck, T. Mikolajick e W. M. Weber, "Functionality-Enhanced Logic Gate Design Enabled by Symmetrical Reconfigurable Silicon Nanowire Transistors," IEEE Transactions on Nanotechnology, pp. 689 - 698, 2015.

[29] mdic, "PADIS," [Online]. Available: http://www.mdic.gov.br/competitividadeindustrial/principais-acoes-de-desenvolvimento-industrial/padis/padis. [Acesso em 1808 2017]. 
[30] J. A. Martino e R. C. Rangel, "TRANSISTOR COM FORMAÇÃO DE FONTE E DRENO INDUZIDA POR EFEITO DE CAMPO ELÉTRICO E SEU MÉTODO DE FABRICAÇÃO". Patente BR 1020150209746 A2, 2015.

[31] R. C. Rangel e J. A. Martino, "Back Enhanced (BE) SOI pMOSFET," Microelectronics Technology and Devices (SBMicro), 2015.

[32] J. P. Colinge, C. W. Lee, A. Afzalian, N. Dehdashti, R. Yan, I. Ferain, P. Razavi, B. O'Neill, A. Blake, M. White, A. M. Kelleher, B. McCarthy e R. Murphy, "SOI gated resistor: CMOS without junctions," IEEE International SOI Conference, 2009.

[33] U. Schwalke, T. Krauss e F. Wessely, "Dopant-Free CMOS on SOI: Multi-Gate Si-Nanowire Transistors for Logic and Memory Applications," ECS Transactions, 53(5), p. 105-114, 2013.

[34] S. Cristoloveanu e S. Williams, "Point-contact pseudo-MOSFET for in-situ characterization of as-grown silicon-on-insulator wafers," IEEE Electron Device Letters, vol. 13, № 2, 1992.

[35] J. A. Martino, M. A. Pavanello e P. B. Verdonck, Caracterização Elétrica de Tecnologia e Dispositivos MOS, São Paulo: Thomson, 2003.

[36] J. P. Colinge e C. A. Colinge, Physics of Semiconductor Devices, Kluwer Academic Publishers, 2002.

[37] H. K. Lim e J. G. Fossum, "Threshold voltage of thin-film Silicon-on-insulator (SOI) MOSFET's," IEEE Transactions on Electron Devices, pp. 1244 - 1251, 1983.

[38] L. M. Camillo, "Estudo do ponto invariante com a temperatura ("Zero Temperature Coefficient") em transistores SOI Mosfet fabricados com tecnologia ultra-submicrométrica.," Tese de Doutorado, 2011.

[39] Z. D. Prijić, S. S. Dimitrijev e N. D. Stojadinović, "The Determination of Zero Temperature Coefficient Point in CMOS Transistors," Microelectronics Reliability, vol. 32, no 6, pp. 769-773, 1992. 
[40] A. A. Osman, M. A. Osman, N. S. Dogan e M. A. Imam, "Zero-temperaturecoefficient biasing point of partially depleted SOI MOSFET's," IEEE Transactions on Electron Devices, vol. 42, oㅜ 9, pp. 1709-1711, 1995.

[41] A. K. Tillmann, F. Wessely e U. Schwalke, "Novel Electrostatically Doped Planar Field-Effect Transistor for High Temperature Applications," ECS Transactions, vol. 64, no 12, pp. 11-24, 2014.

[42] I. M. Filanovsky e A. Allam, "Mutual compensation of mobility and threshold voltage temperature effects with applications in CMOS circuits," IEEE Transactions on Circuits and Systems I: Fundamental Theory and Applications, vol. 48, no 7, pp. 876-884, 2001.

[43] M. Souza, M. A. Pavanello, C. W. Lee, R. T. Doria, I. Ferain, R. Yan, R. Yu, N. D. Akhavan, P. Razavi e J. P. Colinge, "Low Temperature Behavior of Junctionless Multiple Gate nMOSFETs," Proceedings of WOLTE 9, 2010.

[44] J. A. Martino, L. M. Camillo, L. M. Almeida, E. Simoen e C. Claeys, "ZeroTemperature-Coefficient of planar and MuGFET SOI devices," Solid-State and Integrated Circuit Technology (ICSICT), pp. 1753-1756, 2010.

[45] J. M. Rabaey, A. Chandrakasan e B. Nikolic, Digital Integrated Circuits, $2^{\underline{a}}$ ed., Pearson, 2003.

[46] J. P. Colinge, A. Kranti, R. Yan, C. W. Lee, I. Ferain, R. Yu, N. Dehdashti Akhavan e P. Razavi, "Junctionless Nanowire Transistor (JNT): Properties and design guidelines," Solid-State Electronics, Vols. \%1 de \%265-66, pp. 33-37, 2011.

[47] N. D. Akhavan, I. Ferain, P. Razavi, R. Yu e J. P. Colinge, "Random dopant variation in junctionless nanowire transistors," IEEE International SOI Conference, 2011.

[48] L. P. Santos, "Projeto de máscaras com dispositivos e estruturas de teste usando Microwind," São Paulo, Trabalho de conclusão de curso, 2008.

[49] Synopsys TCAD, Sentaurus Device User Guide, Version H-2013.03 ed., 2013. 
[50] H. S. Wong, M. H. White, T. J. Krutsick e R. V. Booth, "Modeling of transconductance degradation and extraction of threshold voltage in thin oxide MOSFET's," Solid-State Electronics, vol. 30, № 9, pp. 953-968, 1987.

[51] L. S. Yojo, J. A. Padovese, R. C. Rangel e J. A. Martino, "Back enhanced (BE) SOI pMOSFET behavior at high temperatures," Symposium on Microelectronics Technology and Devices (SBMicro), 2016.

[52] L. S. Yojo, R. C. Rangel, K. R. A. Sasaki e J. A. Martino, "Back Enhanced (BE) SOI MOSFET under non-conventional bias conditions," Joint International EUROSOI Workshop and International Conference on Ultimate Integration on Silicon (EUROSOI-ULIS), 2017.

[53] L. S. Yojo, R. C. Rangel, K. R. A. Sasaki e J. A. Martino, "Is there a Zero Temperature Bias Point (ZTC) on Back Enhanced (BE) SOI MOSFET?," em S3S Conference, São Francisco, 2017.

[54] L. S. Yojo, R. C. Rangel, K. R. A. Sasaki e J. A. Martino, "Reconfigurable Back Enhanced (BE) SOI MOSFET used to Build a Logic Inverter," Symposium on Microelectronics Technology and Devices (SBMicro), 2017.

[55] J. P. Noel, O. Thomas, M.-A. Jaud, O. Weber, T. Poiroux, C. Fenouillet-Beranger, P. Rivallin, P. Scheiblin, F. Andrieu, M. Vinet, O. Rozeau, F. Boeuf, O. Faynot e A. Amara, "Multi-VT UTBB FDSOI Device Architectures for Low-Power CMOS Circuit," IEEE Transactions on Electron Devices, vol. 58, no 8, pp. 2473-2482, 2011. 


\section{Apêndice A}

Exemplo de código usado para simular o BE SOI MOSFET.

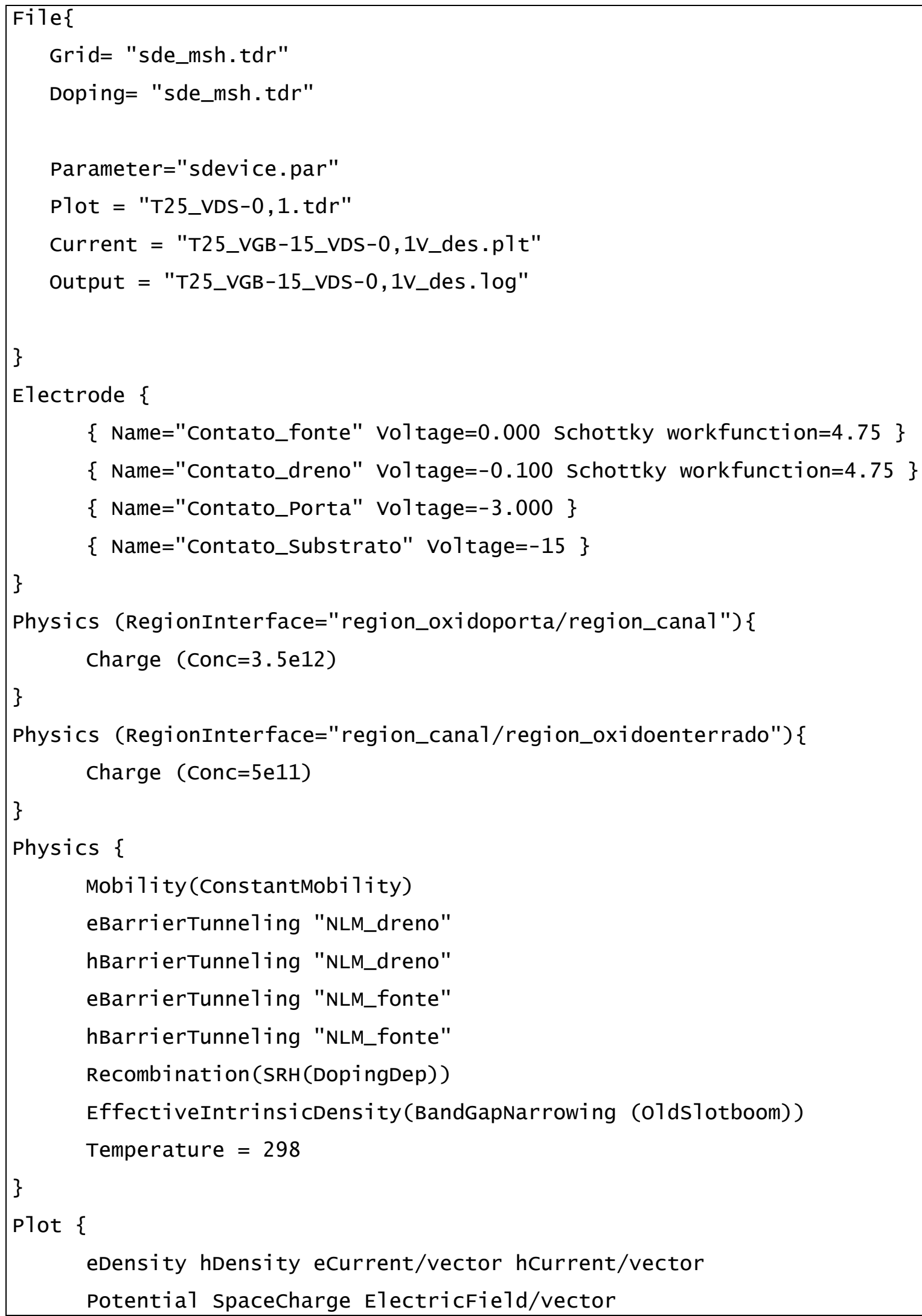




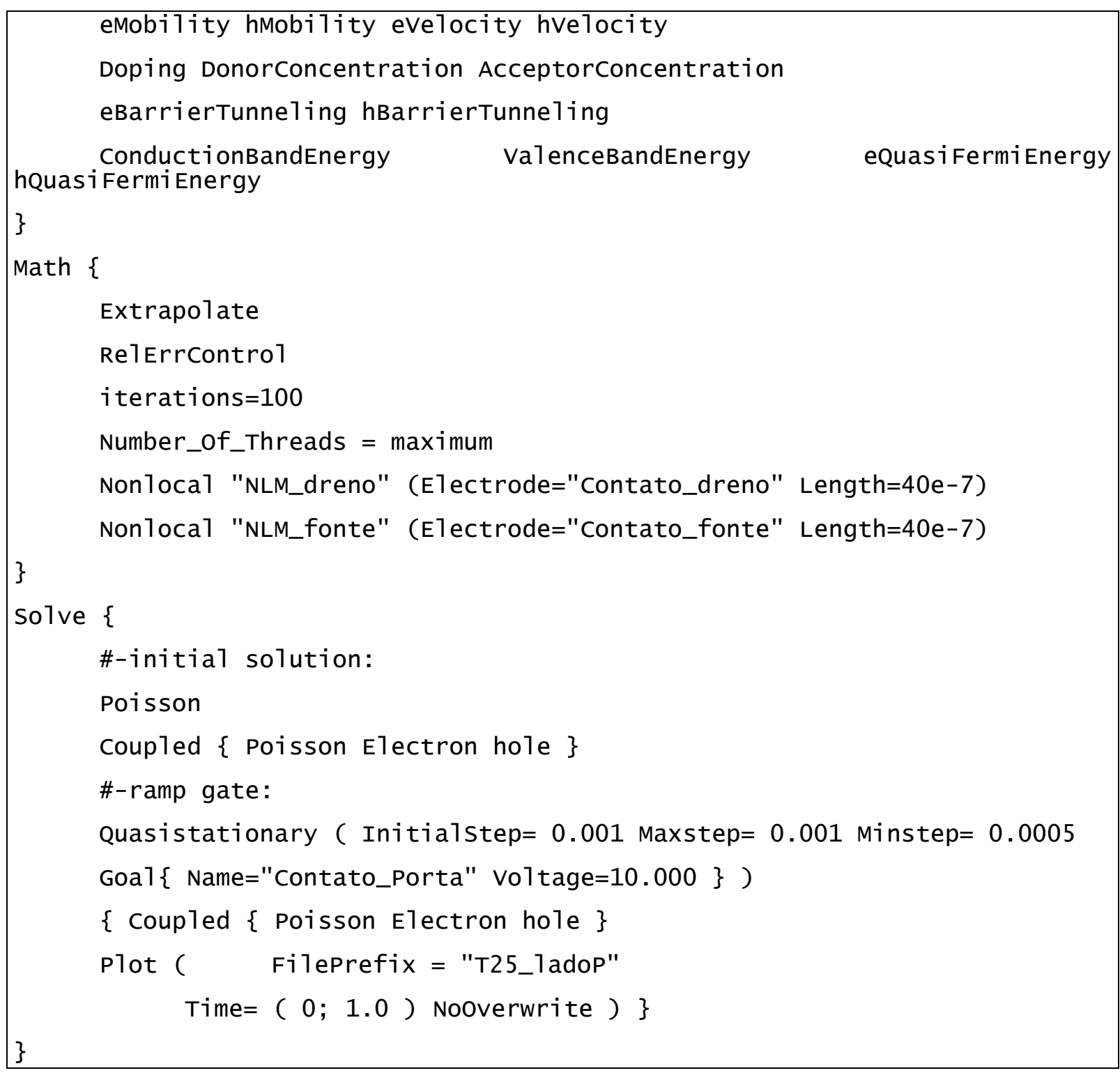

